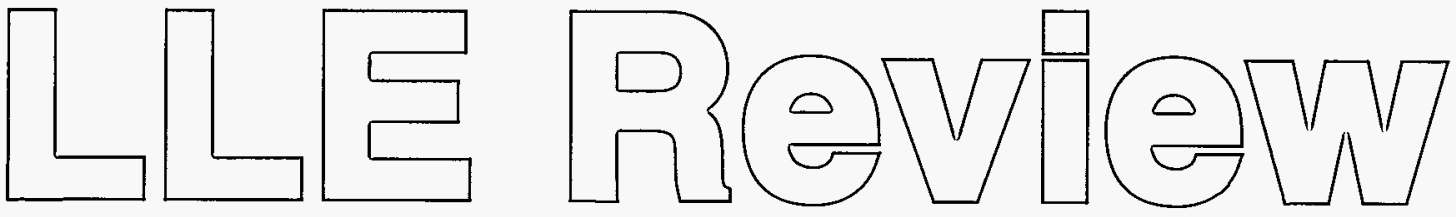

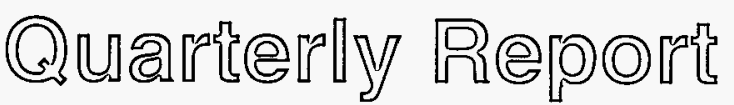

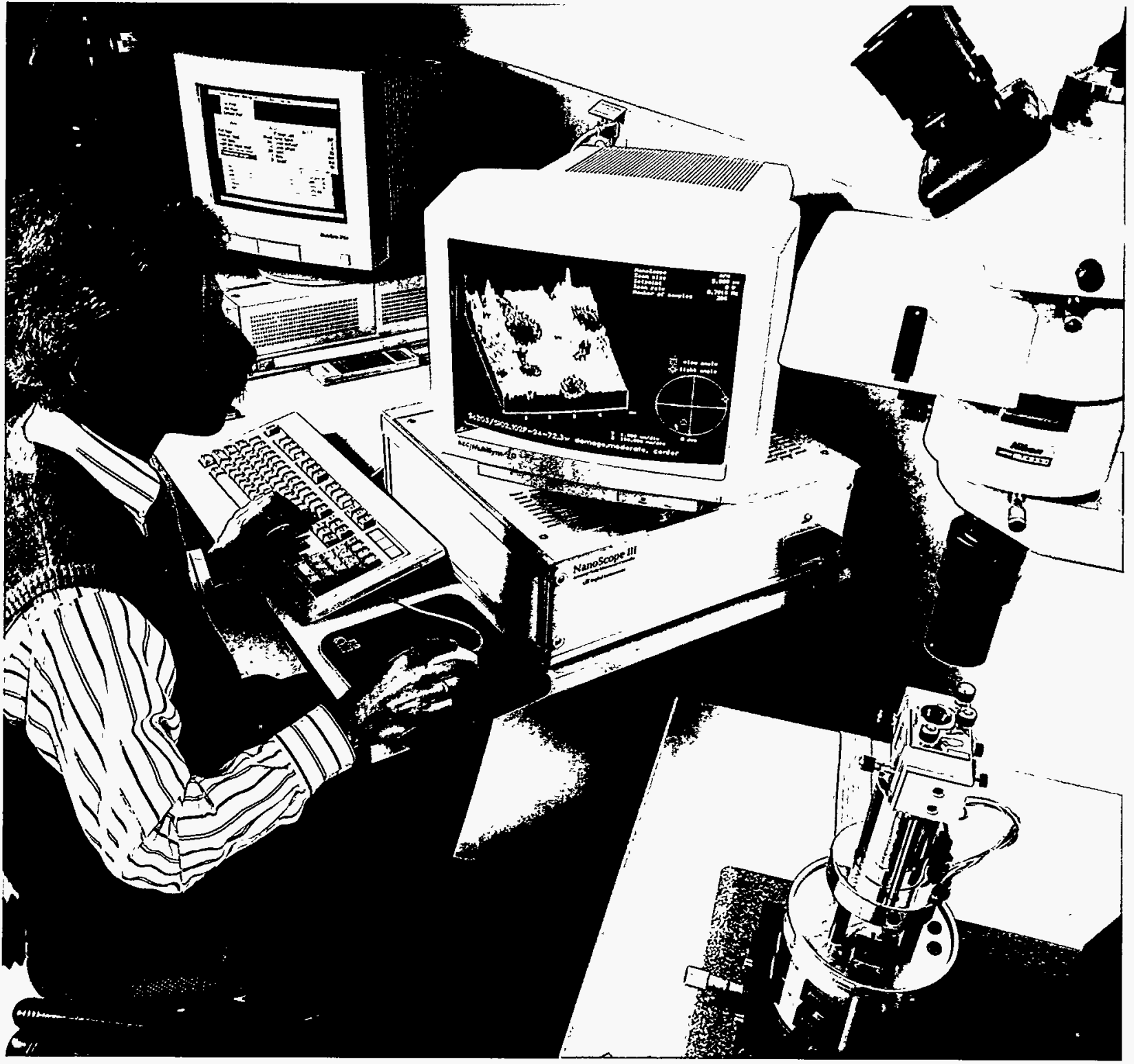




\section{About the Cover:}

Semyon Papernov, a scientist in the Optical Technology Group, uses a Digital Instruments Nanoscope III atomic force microscope (AFM) operated in contact mode to evaluate the morphology of $3 \omega$ laser-damage features on a multilayer, quarter-wave-stack OMEGA transport $\mathrm{HR}$ coating made from $\mathrm{HfO}_{2}$ and $\mathrm{SiO}_{2}$. Surface mapping of damaged and undamaged sites on production witness pieces by atomic force microscopy has shown that nodular growth defects, long considered to be the dominant laser-damage driver in dielectric thin films, can remain unaffected under 351-nm irradiation conditions, while other defect-driving mechanisms dominate damage crater formation in the immediate vicinity of the nodules.

This report was prepared as an account of work conducted by the Laboratory for Laser Energetics and sponsored by New York State Energy Research and Development Authority, the University of Rochester, the U.S. Department of Energy, and other agencies. Neither the above named sponsors, nor any of their employees, makes any warranty, expressed or implied, or assumes any legal liability or responsibility for the accuracy, completeness, or usefulness of any information, apparatus, product, or process disclosed, or represents that its use would not infringe privately owned rights. Reference herein to any specific commercial product, process, or service by trade name, mark, manufacturer, or otherwise, does not necessarily constitute or imply its endorsement, recommendation, or favoring by the United States Government or any agency thereof or any other sponsor. Results reported in the LLE Review should not be taken as necessarily final results as they represent active research. The views and opinions of authors expressed herein do not necessarily state or reflect those of any of the above sponsoring entities.

The work described in this volume includes current research at the Laboratory for Laser Energetics, which is supported by New York State Research and Development Authority, the University of Rochester, the U.S. Department of Energy Office of Inertial Confinement Fusion under Cooperative Agreement No.

Printed in the United States of America Available from

National Technical Information Services

U.S. Department of Commerce

5285 Port Royal Road

Springfield, VA 22161

Price codes: Printed Copy A04

Microfiche A01
DE-FC03-92SF19460, and other agencies.

For questions or comments, contact Kenneth L. Marshall, Editor, Laboratory for Laser Energetics, 250 East River Road, Rochester, NY 14623-1299, (716) 275-8247. 


\section{DISCLAIMER}

Portions of this document may be illegible in electronic image products. Images are produced from the best available original document. 


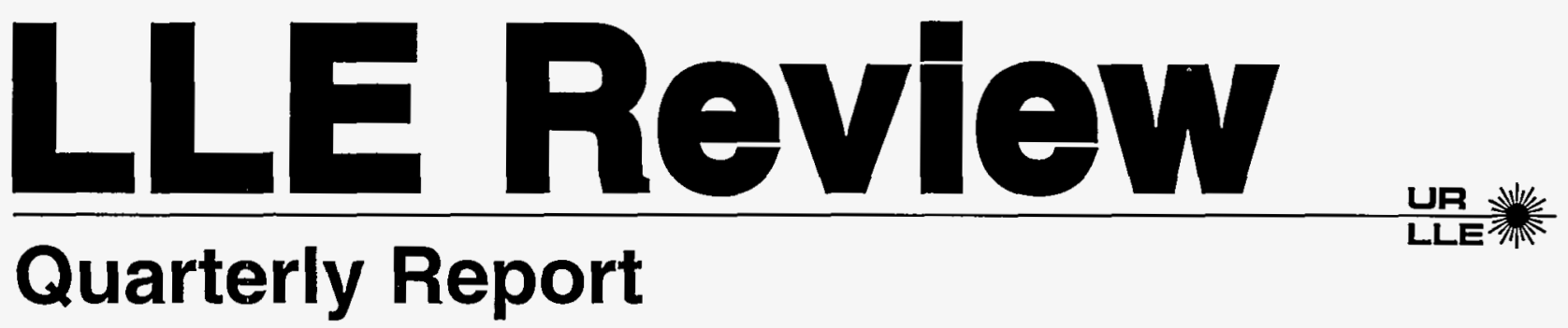

\section{Contents}

In Brief iii

An Intrinsic Laser-Damage Mechanism in Next-Generation OMEGA UV Multilayer Coatings

The OMEGA Laser Pulse-Shaping System 56

Direct-Drive Irradiation Uniformity for the NIF 62

Direct Measurements of Terminal-Level

Lifetime in Nd:YLF 71

Light-Emitting Porous Silicon: Materials Science, Properties, and Device Applications

Time-Dependent Reflection and Surface Temperatures during $\mathrm{CO}_{2}$ Laser Irradiation of Dental Hard Tissues with $100-\mu$ s Pulses

Publications and Conference Presentations 


\section{In Brief}

This volume of the LLE review, covering the period of January-March 1995, contains articles on the evaluation of the mechanism for laser damage in OMEGA UV multilayer coatings using a combination of conventional laser-damage characterization methods and atomic force microscopy; a dual-amplitude, fiber-coupled waveguide integrated-optic modulation device for generating temporally shaped optical pulses in OMEGA; a proposal for modifying the indirect-drive irradiation geometry of the National Ignition Facility (NIF) to provide the additional flexibility for performing direct-drive experiments; direct measurements of terminal-level lifetime in several different Nd:YLF laser media; an overview of the materials science issues, basic mechanisms, and potential device applications for light-emitting porous silicon; and a study of the time-dependent reflection and surface temperatures for laser-irradiated dental hard tissue at two $\mathrm{CO}_{2}$ laser wavelengths.

Highlights of the research presented in this issue are

- Submicron, lateral-sized craters that develop independently of the presence of micron-scale growth nodules and whose number density follows the intensity profile of the full laser beam appear to be the dominant damage feature in UV high-reflector coatings. Coupled with the observation that the smallest measured craters allow for starting absorber sizes of $<10 \mathrm{~nm}$, these results point toward randomly distributed nano-cluster absorbers as the sources involved in the energy transfer from the optical field to the porous film medium.

- An optical pulse-shaping system employing integrated-optic amplitude modulators operated in series and fabricated on a single, fiber-coupled $\mathrm{LiNbO}_{3}$ waveguide has been designed. This system is capable of meeting future pulse-shaping requirements for the OMEGA laser. In recent operational testing, this system has demonstrated the ability to produce shaped optical pulses with 50- to 100-ps structure over a pulse envelope of several nanoseconds.

- Highly uniform irradiation for direct-drive experiments can be produced by NIF if its design is modified to allow one-half of the beams to be redirected to new ports closer to the equator of the target chamber and if 2-D SSD with polarization dispersion is implemented. The tolerances for energy imbalance among the beams, beam mispointing, and errors in target positioning will depend on how much long-wavelength nonuniformity the target can accept without a serious degradation in performance.

- The terminal-level lifetimes for four different Nd:YLF samples as determined by small-signal-gain and transient excited state absorption measurements were found to be considerably longer than the pulse lengths encountered in mode-locked laser operation and amplification of up to nanosecond pulses. Because the terminal-level-laser lifetime in these media approaches the length of a common $Q$-switched laser pulse, simple analytical models are inadequate to account for terminal-level relaxation during amplification of such pulses, and numerical solutions will be required in order to calculate energy-extraction performance. 
- Room-temperature photoluminescence with an efficiency between $0.1 \%$ and $10 \%$ has been demonstrated in porous silicon, and its luminescence spectrum, intensity, and lifetime have been shown to be highly sensitive to growth and processing parameters. The ability to vary the peak of the photoluminescence spectrum from the blue/violet to wavelengths past $1.5 \mu \mathrm{m}$ makes these materials of interest in the fabrication of light-emitting devices (LED's) operating over the same wavelength range.

- The first experimental evidence of time-dependent reflection in dental hard tissue irradiated with a 9.6- $\mu \mathrm{m}, \mathrm{CO}_{2}$ laser has been observed and is related to the temperature dependence of the absorption coefficient using known relationships between surface reflection and high absorption coefficients. These results indicate that morphological surface modifications in dental hard tissue can be fine-tuned by making an appropriate choice of wavelength and pulse duration, which would allow optimization of the laser-irradiation conditions for the reduction of dental decay.

Kenneth L. Marshall

Editor 


\section{An Intrinsic Laser-Damage Mechanism in Next-Generation OMEGA UV Multilayer Coatings}

Laser-induced damage has always been and remains as vexing as it is expensive. Despite a quarter century of work, the problem has not been eliminated or even fully defined-only the technical limits have been increased. Although dielectric thin films have improved over the last 25 years, they remain especially difficult subjects to deal with owing to the multiparameter nature of the nonequilibrium deposition methods used in their preparation. As new analytical methods and tools become available, however, better insight into the laser-damage enigma is gained.

The importance of nodular film-growth defects in originating laser damage was one such key insight that was recently offered through a series of pioneering papers by the Optical Coatings Group at Lawrence Livermore National Laboratory. ${ }^{1-4}$ These results were achieved through a combination of (1) analytical modeling of laser-field effects in and around growth nodules; (2) mapping of these growth nodules under atomic-force microscopy; and (3) physical analysis of the nodules by focused-ion-beam cross sectioning and Auger spectroscopy. In conjunction with conventional laser-damagethreshold measurements, this approach yielded the broad conclusion that nodular growth defects are the dominant laserdamage drivers in thin films. From a systems design viewpoint, nodules became the coating affliction to be eradicated for laser-damage thresholds to improve.

As results for the fundamental and second-harmonic-wavelength laser-damage characteristics of multilayer coatings were being disseminated, prototype development and actual production of OMEGA Upgrade transport coatings were proceeding at LLE. In this article we report our observations on laser damage in these $3 \omega(351-\mathrm{nm})$ high reflectors (HR's), obtained by combining conventional $3 \omega$ laser-damage investigations with mapping of damaged and undamaged sites on production witness pieces by atomic force microscopy. These findings differ from the earlier ones for 1054-nm and 527-nm conditions in that the nodules are capable of surviving fluences that cause crater formation by other mechanisms. These mecha- nisms confine crater origination to the top two layers of the multilayer, high-reflector stack.

\section{Samples and Experimental Conditions}

Two types of oblique-incidence, $3 \omega$ high reflectors were analyzed: (1) a $46^{\circ}$ angle-of-incidence, 37 alternating quarterwave stack $\mathrm{HR}$ made from $\mathrm{HfO}_{2}$ and $\mathrm{SiO}_{2}$, with the first and third $\mathrm{HfO}_{2}$ layers replaced by $\mathrm{Sc}_{2} \mathrm{O}_{3}$ to make the coating strippable; and (2) a $50^{\circ}$ angle-of-incidence, 53 alternating quarter-wave stack of $\mathrm{Sc}_{2} \mathrm{O}_{3}$ and $\mathrm{SiO}_{2}$, with 47 layers of exact quarter-wave optical thicknesses and the top six layers adjusted in thickness for reduced electric-field intensity at the layer interfaces. Both coatings were deposited by conventional e-beam methods. One of the primary goals in the deposition process was to minimize the formation of growth nodules.

Witness pieces from these production coatings were irradiated at the respective angles using frequency-tripled, 0.7-ns pulses from a Nd:glass slab laser. At optimized conversion efficiency and under weak focusing by a $10-\mathrm{m}$ focal-length silica lens, fluences up to $14 \mathrm{~J} / \mathrm{cm}^{2}$ within a $600-\mu \mathrm{m}$ spot diameter were delivered to the test piece. Damage was identified by dark-field microscopy (110-times magnification) as an agglomeration of very fine, low-light-level scattering sites (nebula). The damage thresholds in 1-on-1 irradiation mode ( $p$-polarization, scaled to $0^{\circ}$ incidence for systems design engineering convenience) were $5.6 \pm 0.2 \mathrm{~J} / \mathrm{cm}^{2}$ in the case of the $\mathrm{HfO}_{2} / \mathrm{SiO}_{2}$ system, and $5.4 \pm 0.1 \mathrm{~J} / \mathrm{cm}^{2}$ for $\mathrm{Sc}_{2} \mathrm{O}_{3} / \mathrm{SiO}_{2}$. Sites later mapped by atomic force microscopy experienced damage at $\sim 5 \%$ above threshold (defined as "weak" damage sites) and at 15\%-20\% above threshold fluences (defined as "moderate" damage sites).

Analysis of both irradiated and unirradiated (control) sites was carried out using a Nanoscope III (Digital Instruments, Inc.) atomic force microscope (AFM) operated in contact mode. "Oxide-sharp" $\mathrm{Si}_{3} \mathrm{~N}_{4}$ probes proved adequate for resolving both nanometer-scale film morphology and any laser-modified structures, although rapid wear required fre- 
quent probe replacement. Typical "stickiness" between the sample and probe of dielectric oxide films during scans was counteracted by making adjustments in scanning speeds and feedback loop parameters. The contact force was routinely minimized to ensure an unmodified scan area during repeated scans. Adjacent, $5-\mu \mathrm{m} \times 5-\mu \mathrm{m}$ sections were mapped with the goal of accumulating statistically significant numbers of various damage features, such as cracks, craters, or domes. For a given sample and irradiation condition, this number is of the order of 100 for each irradiated site. Each feature observed was subsequently analyzed in high-resolution closeups.

\section{Results and Discussion}

\section{Morphology of Unirradiated Films}

In both the $\mathrm{HfO}_{2} / \mathrm{SiO}_{2}$ and $\mathrm{Sc}_{2} \mathrm{O}_{3} / \mathrm{SiO}_{2}$ stacks, the terminating (top) layer is the respective high-refractive-index medium (i.e., $\mathrm{HfO}_{2}$ or $\mathrm{Sc}_{2} \mathrm{O}_{3}$ ). Figures 62.1 and 62.2 show the typical oxide-film columnar structures for these materials, with 25 - to 40-nm grain size and 3.3- to 3.6-nm vertical rms roughness. Taking into account an AFM probe tip radius $R_{\text {tip }} \sim 10 \mathrm{~nm}$ (according to manufacturer specifications) and the recently reported image-distortion criterion, ${ }^{5}$ i.e., $R_{\text {grain }} / R_{\text {tip }}<2$, we find our imaged grain size to be overestimated. The magnitude of this error, however, is not large, as we were able to resolve the 10 -nm-scale granular structure of $\mathrm{Ta}_{2} \mathrm{O}_{5}$ monolayer films using the same probes. ${ }^{6}$ These features are less than half the scale length of the $\mathrm{HfO}_{2}$ and/or $\mathrm{Sc}_{2} \mathrm{O}_{3}$ characteristic grains.

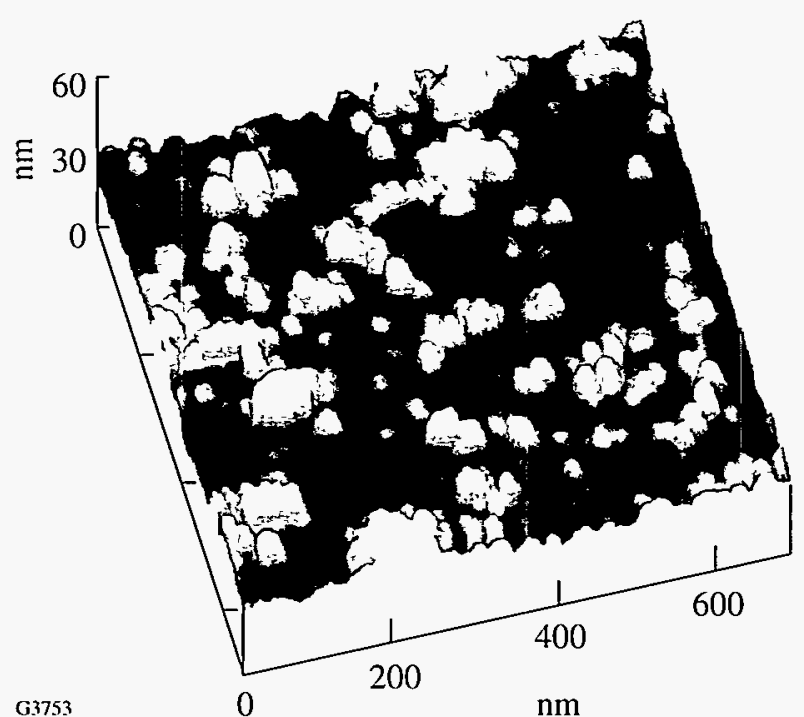

Figure 62.1

Columnar structure of the $\mathrm{HfO}_{2}$ top surface $(700 \times 700-\mu \mathrm{m}$ scan $)$ of a $\mathrm{HfO}_{2} /$ $\mathrm{SiO}_{2}$ multilayer $\mathrm{HR}$ stack.

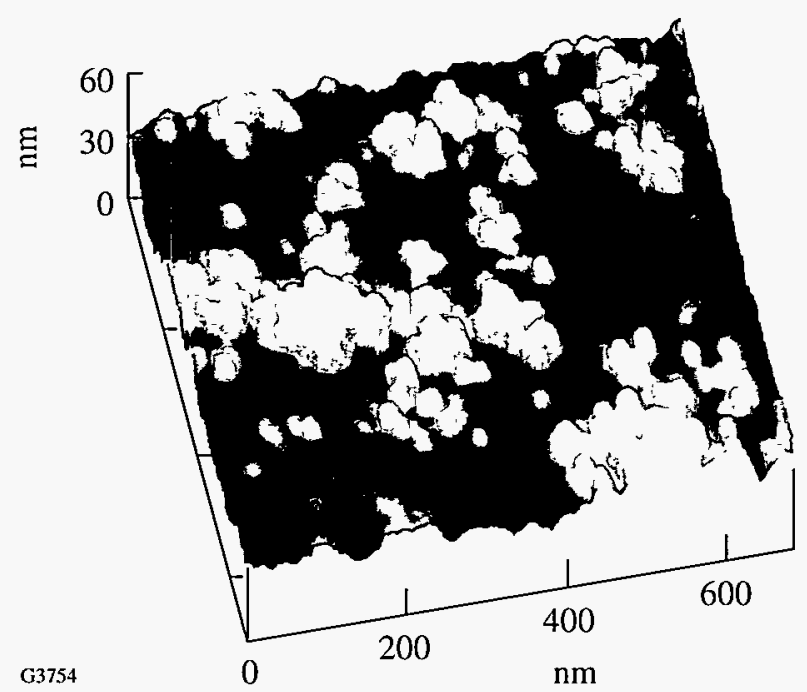

Figure 62.2

Columnar structure of the $\mathrm{Sc}_{2} \mathrm{O}_{3}$ top surface $(700 \times 700-\mu \mathrm{m}$ scan $)$ of $\mathrm{S} \mathrm{Sc}_{2} \mathrm{O}_{3}$ / $\mathrm{SiO}_{2}$ multilayer $\mathrm{HR}$ stack with reduced $E$-field design.

\section{Laser-Induced Morphology}

In both $\mathrm{HfO}_{2} / \mathrm{SiO}_{2}$ and $\mathrm{Sc}_{2} \mathrm{O}_{3} / \mathrm{SiO}_{2}$ coatings, craters on the scale of $200 \mathrm{~nm}$ to $700 \mathrm{~nm}$ across and up to $130 \mathrm{~nm}$ deep are the main laser-damage features (Figs. 62.3 and 62.4). Their spatial correlation with the incident beam-fluence profile recorded in a sample equivalent plane by a CID camera is weak or nearly nonexistent. Under weakly damaging conditions,

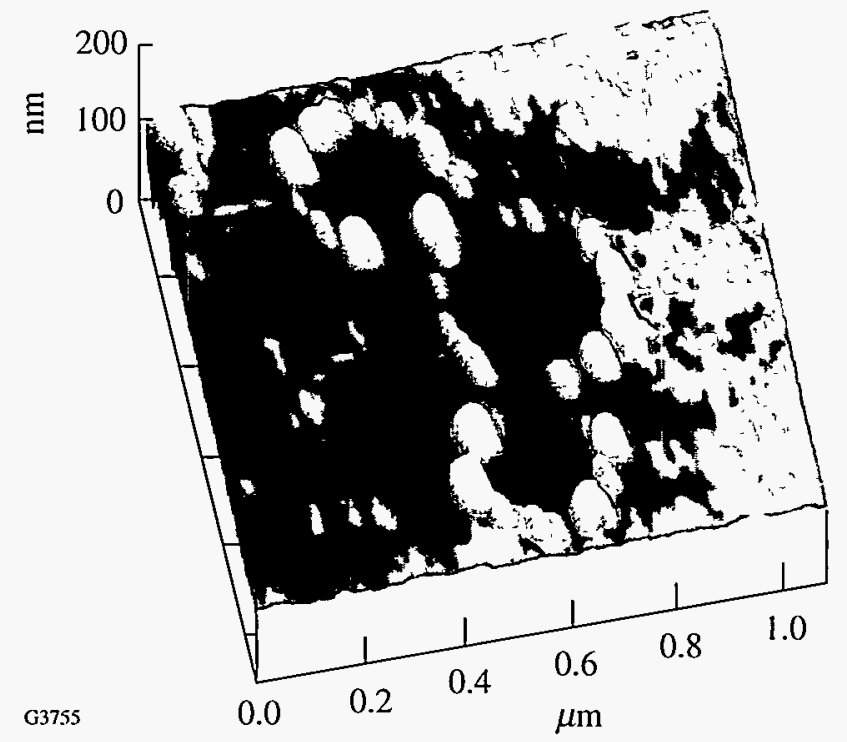

Figure 62.3

Conventional laser-damage craters formed by $351-\mathrm{nm}$ radiation in the $\mathrm{HfO}_{2} /$ $\mathrm{SiO}_{2}$ film. The area shown corresponds to the periphery of a moderatedamage site. 


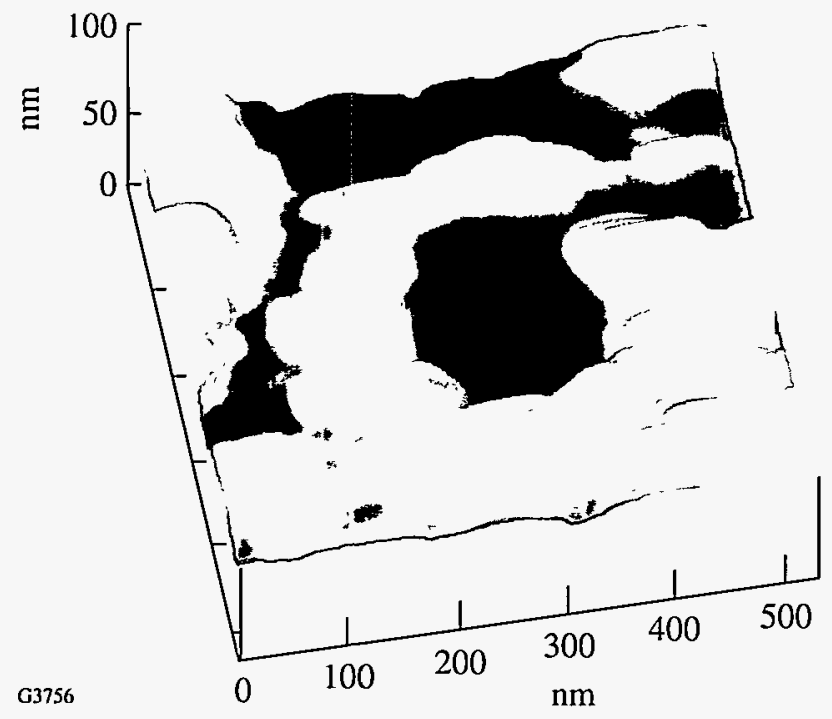

Figure 62.4

Conventional laser-damage crater formed by $351-\mathrm{nm}$ radiation in the $\mathrm{Sc}_{2} \mathrm{O}_{3} /$ $\mathrm{SiO}_{2}$ film. The crater is $38 \mathrm{~nm}$ deep and, on average, $300 \mathrm{~nm}$ in diameter.

crater-to-crater average distances are $2.4 \mu \mathrm{m}$ for $\mathrm{HfO}_{2} / \mathrm{SiO}_{2}$ and $2.7 \mu \mathrm{m}$ for $\mathrm{Sc}_{2} \mathrm{O}_{3} / \mathrm{SiO}_{2}$. In agreement with our earlier findings on 1054-nm laser-irradiated monolayer films, ${ }^{6}$ such craters develop independently of whether or not micron-scale growth nodules are present. Figure 62.5 demonstrates that growth nodules and inclusions can remain unaffected, even under 351-nm irradiation, while other defect-driving mecha-

(a)

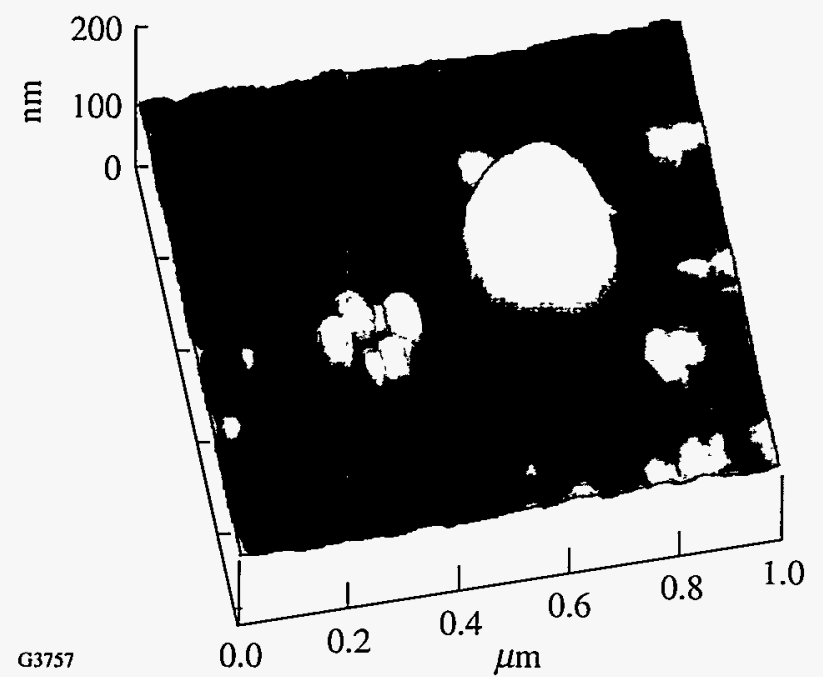

nisms dominate crater formation in the nodules' immediate vicinity. Up to now, we have been unable to offer similar evidence for 1054-nm HR stacks. Within that constraint, the current evidence suggests that growth nodules are not always the dominating cause of damage. We have, in fact, no AFM evidence or visible-light microscopy record for any observable feature that could be used as a landmark for predicting the occurrence of these craters.

Closer analysis of these craters offers some interesting conclusions regarding their potential source. In agreement with LLNL findings from $1981,{ }^{7}$ under near-threshold conditions the majority of craters are very shallow, originating in either or both of the top two layers; i.e., $29 \%$ of $\mathrm{HfO}_{2} /$ $\mathrm{SiO}_{2}$ craters and $17 \%$ of $\mathrm{Sc}_{2} \mathrm{O}_{3} / \mathrm{SiO}_{2}$ craters are less than $20 \mathrm{~nm}$ deep. If a compact absorber is postulated as the damage originator, one can deduce immediately from this crater depth that such an absorber would have to be limited in extent to $\sim 10 \mathrm{~nm}$.

Modern AFM tools now permit greater insight into crater cross-sectional features than were observed 14 years ago. We have found many instances in which a conventional, coneedged crater is superimposed on a second, deeper crater (see Fig. 62.6). These complex craters occur mainly in $\mathrm{Sc}_{2} \mathrm{O}_{3} / \mathrm{SiO}_{2}$ with an abundance level of $37 \%$. Conventional craters show conical walls that are normally smooth and without any grain

Figure 62.5

(a) Crater formed in the vicinity of an unaffected nodular growth defect in the $\mathrm{HfO}_{2} / \mathrm{SiO}_{2}$ film. The columnar structure of the nodule surface is masked by the saturating dome height $(\sim 200 \mathrm{~nm})$ relative to the only $15-\mathrm{nm}$ peak-to-valley excursions characteristic for the columnar structure. (b) High-pass filtered version of the image in Fig. 62.5(a), highlighting the columnar structure and nodular nature of the defect. 
(a)

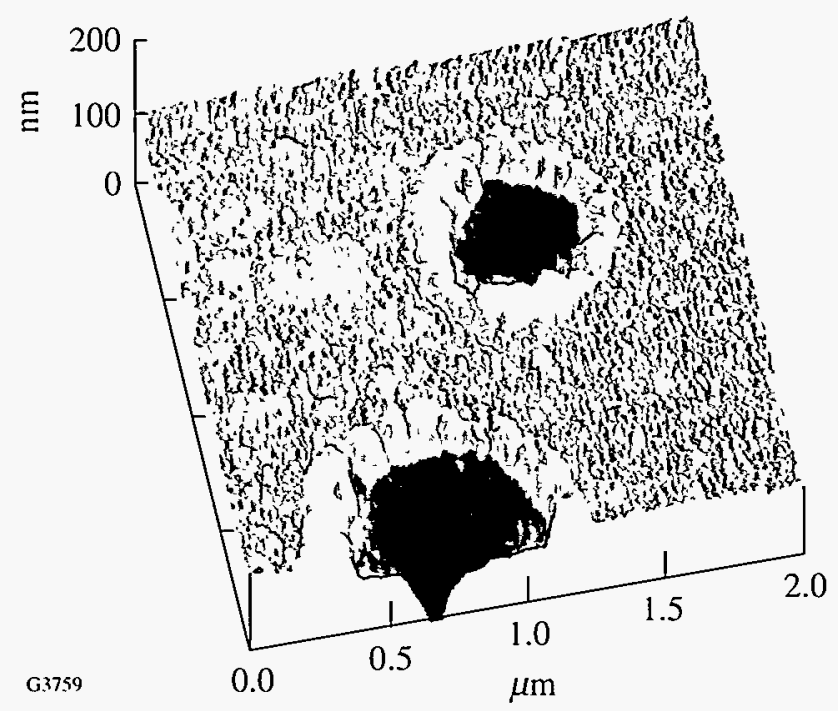

(b)

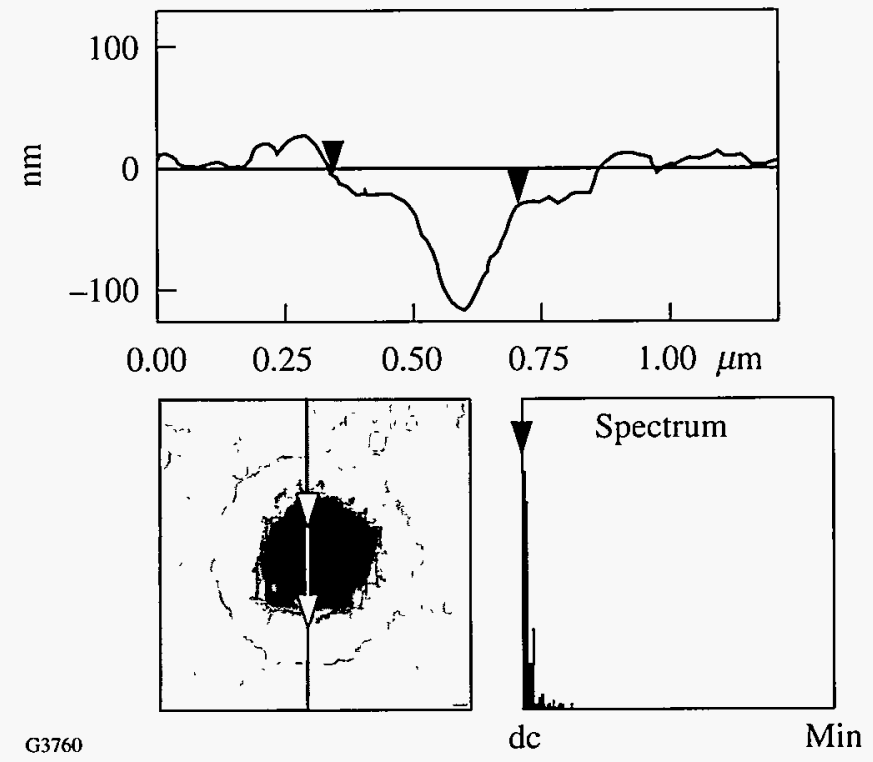

Figure 62.6

(a) Complex crater(s) in the $\mathrm{Sc}_{2} \mathrm{O}_{3} / \mathrm{SiO}_{2}$ film show the preserved columnar structure within the outer, larger-diameter cone. This indicates that melting remains localized within the inner cone volume, i.e., the $\mathrm{SiO}_{2}$ layer. (b) Cross section through the complex crater in the $\mathrm{Sc}_{2} \mathrm{O}_{3} / \mathrm{SiO}_{2}$ film. The double-cone structure is clearly pronounced here. as well as the 32.8 -nm step formed by the outer cone.

structure with ridges elevated above the film surface average (Fig. 62.7). In complex craters, the inner, deeper cone also shows smooth, glassy walls, while the outer cone and the horizontal interface clearly preserve columnar structure. Although the horizontal step between the outer and inner cones occurs reproducibly at $32.8 \pm 0.3 \mathrm{~nm}$ from the nominal top surface average ( 36 craters were sampled for this value by AFM determination) and intuitively corresponds to the $\mathrm{Sc}_{2} \mathrm{O}_{3}$ ' $\mathrm{SiO}_{2}$ interface, the top-layer $\left(\mathrm{Sc}_{2} \mathrm{O}_{3}\right)$ physical thickness determined during evaporation by quartz-microbalance and optical-monitoring methods is supposed to be $25.4 \pm 3 \mathrm{~nm}$. We ascribe this discrepancy to the uncertainty in material-density and refractive-index values for evaporated thin films when extracting physical thickness data from mass deposition and interference numbers.

Without detailed simulation, it remains unclear whether the observed double-cone damage is driven by starting events in the first or the second layer. In each scenario, the large difference in melting temperature between $\mathrm{SiO}_{2}\left(T_{m}=1753^{\circ} \mathrm{C}\right)$ and $\mathrm{Sc}_{2} \mathrm{O}_{3}\left(T_{m} \sim 2400^{\circ} \mathrm{C}\right)$ is essential in explaining the observed morphology. As the $\mathrm{SiO}_{2}$ crater walls yield no columnarstructure indicators, we can assume that melting has occurred in the $\mathrm{SiO}_{2}$ layer. Conversely, the columnar spatial frequencies typical of the unirradiated film are maintained around the
$\mathrm{Sc}_{2} \mathrm{O}_{3}$ crater walls and ridges, which rules out large-scale melting in that layer [Fig. 62.6(a)]. Temperatures insufficient for melting in $\mathrm{Sc}_{2} \mathrm{O}_{3}$ are adequate to produce thermal expansion and possible buckling of the 25- to 32-nm top layer that, with or without further pressure from the underlying, melted $\mathrm{SiO}_{2}$ layer, severed along grain boundaries and "popped out." In the event that the initiating absorber was lodged in the second $\left(\mathrm{SiO}_{2}\right)$ layer, the melting of that layer preceded the thermal distortion of the top layer. If the absorber was instead in the top layer, heat from that layer must first propagate across the layer boundary at a flow rate that supports melting of $\mathrm{SiO}_{2}$ columns before the top layer pops out. On the basis of postmortem evidence alone, the dominant or most likely mechanism cannot be determined with any certainty.

Superimposed, double-crater damage is absent in the $\mathrm{HfO}_{2} /$ $\mathrm{SiO}_{2}$ system. Conventional craters become more numerous and, if sufficiently adjacent to one another, merge as the level of damage increases in this system (Fig. 62.8). In spite of earlier reports to the contrary ${ }^{8}$ that rank quarter-wave layers of $\mathrm{SiO}_{2}$ at nearly a factor of 2 higher in 351-nm thresholds than equivalent $\mathrm{HfO}_{2}$ layers of the same thickness, all merging craters in our samples originate in the $\mathrm{SiO}_{2}$ layer after exposure to fluences above threshold. This issue will be discussed in detail in the next section. 


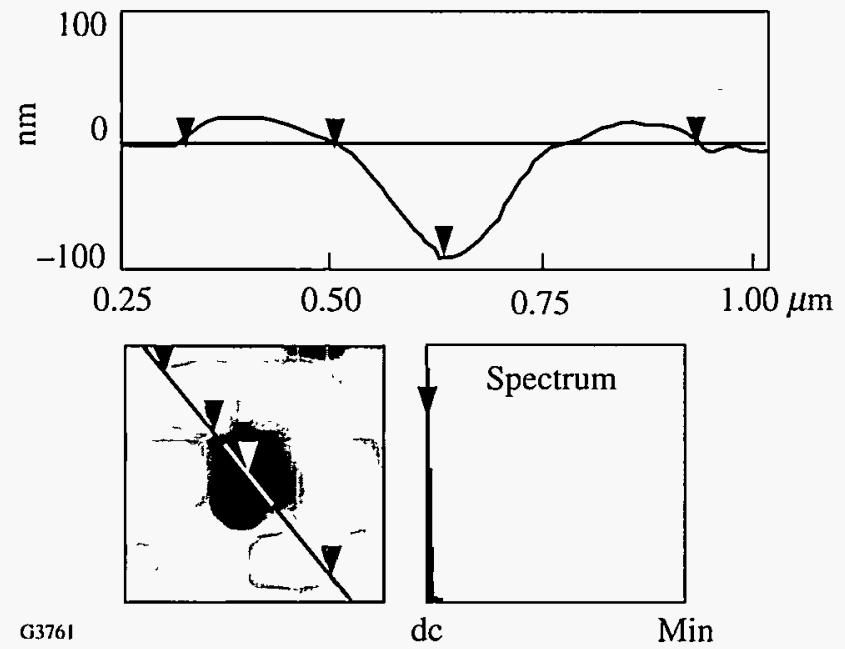

Figure 62.7

Cross section through the 46-nm-deep conventional crater in the $\mathrm{Sc}_{2} \mathrm{O}_{3} /$ $\mathrm{SiO}_{2}$ film. Smooth, glassy walls offer evidence of melting as part of the damage process.

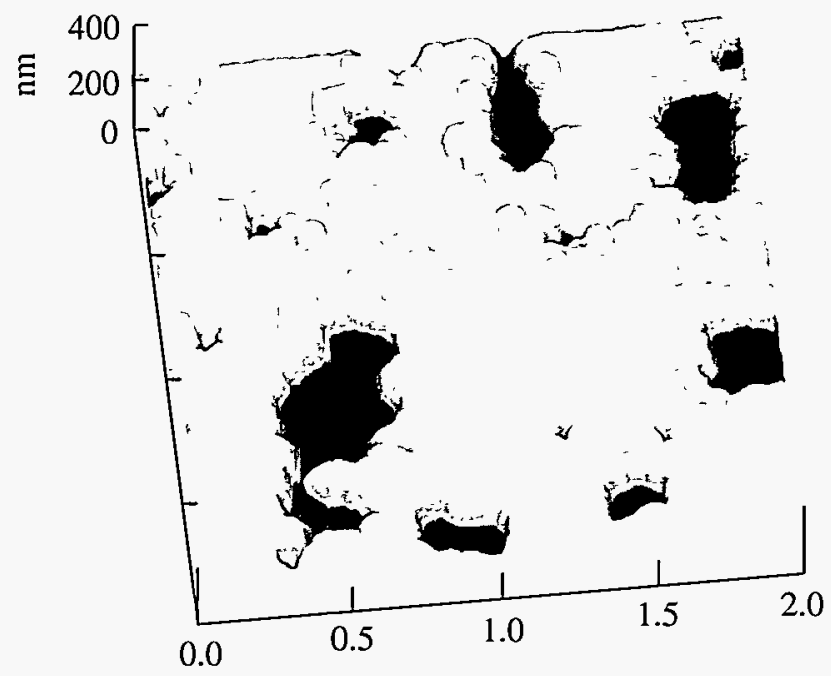

G3762

$\mu \mathrm{m}$

Figure 62.8

Merging craters in the central portion of a moderate-damage event in the $\mathrm{HfO}_{2} / \mathrm{SiO}_{2}$ film.

\section{Crater-Depth Distribution}

For both material systems, crater-depth distributions were recorded for three irradiation conditions: (1) weak damage, attained at fluences $\sim 5 \%$ above the threshold average; (2) moderate damage at $15 \%-20 \%$ above threshold; and (3) the periphery condition of moderate damage, estimated to correspond to a fluence condition intermediate between (1) and (2). Damage-crater histograms were assembled using 10-nm-wide bins as a matter of convenience, with the under- standing that for other bin sizes the crater-count distribution would vary. The arguments in the following sections are therefore not focused on exact depths, but rather on trends.

a. $\mathrm{HfO}_{2} / \underline{\mathrm{SiO}}_{2}$. The weak-damage results summarized in Fig. 62.9 show that craters originate in the first or second layer only, with the majority originating in the 46-nm-thick $\mathrm{HfO}_{2}$ layer. The distribution peaks at a depth near $20 \mathrm{~nm}$ and shifts to greater depths with increasing fluence. At the periphery of moderate damage (Fig. 62.10), two peaks appear in the distribution at $30 \mathrm{~nm}$ and $80 \mathrm{~nm}$, respectively; in the center of moderate damage, craters appear only infrequently in the $\mathrm{HfO}_{2}$ layer, while the distribution peak centers around $75 \mathrm{~nm}$ (Fig. 62.11). In the latter case, a few damage events originate near the interface between the second and third layers, but none were observed protruding into the next $\mathrm{HfO}_{2}$ layer. Concurrent with this shift of craters to greater depths is an increase in both their density (more than an order of magnitude higher for moderate damage than for near-threshold damage) and their tendency to merge.

Energy-balance considerations suggest that the amount of energy near threshold needed for removal of a cone volume (anticrater) is met predominantly by absorbers closer to the surface. We assume here that (1) the crater bottom is either at or very near the location of the absorbing defect, and (2) the defect characteristics and depth do not vary significantly from one AFM mapping area to the next. At energy values further above threshold, deeply located defects absorb sufficient energy to remove material and form a crater, while those closer to the surface not only cause material removal but also dissi-

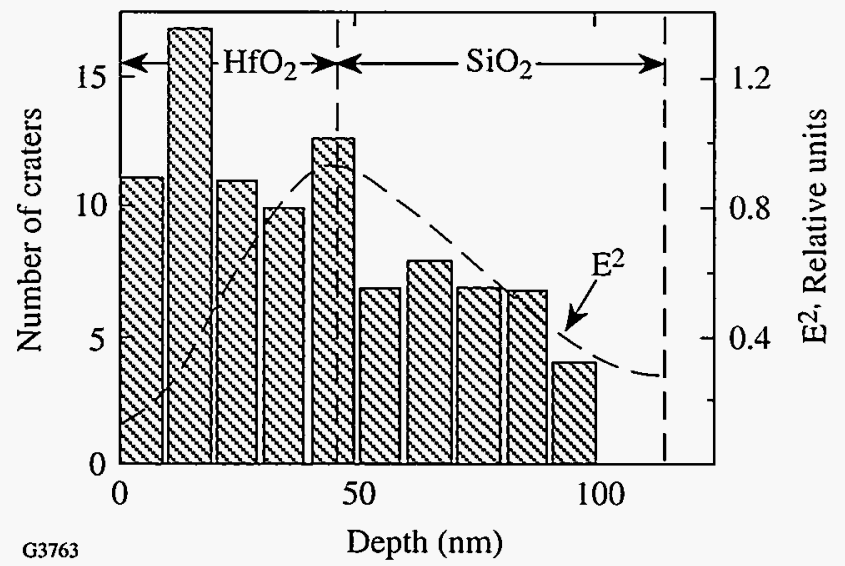

Figure 62.9

Crater-depth distribution (histogram) for weak-damage conditions ( $-5 \%$ above threshold fluence) in the $\mathrm{HfO}_{2} / \mathrm{SiO}_{2}$ system. The majority of craters originate within the top $\mathrm{HfO}_{2}$ layer. 


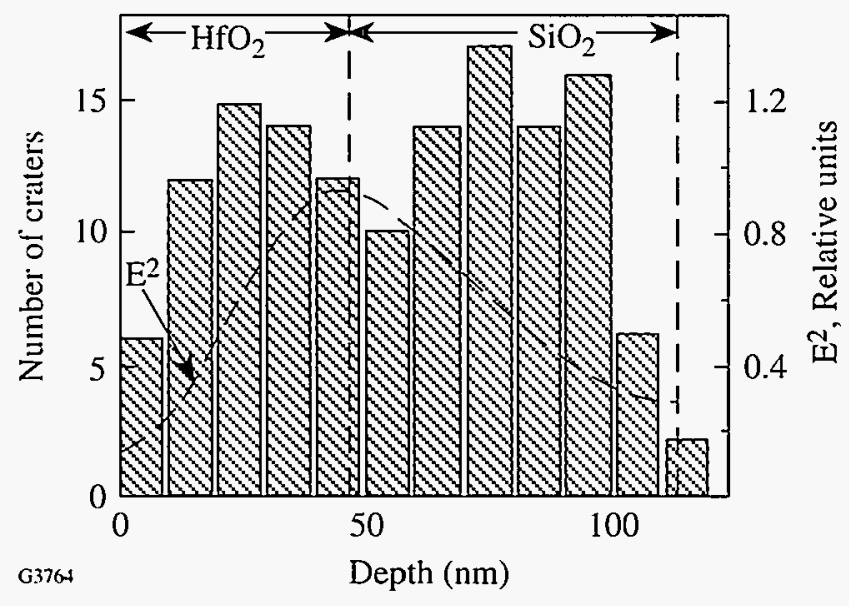

Figure 62.10

Crater-depth distribution (histogram) for moderate-damage (periphery) conditions in the $\mathrm{HfO}_{2} / \mathrm{SiO}_{2}$ system. The second peak at 80 -nm depth may be attributed to the combined effects of excess energy (relative to weak-damage conditions) and heat propagation from the higher-melting-point $\mathrm{HfO}_{2}$ layer to the lower-melting-point $\mathrm{SiO}_{2}$ layer.

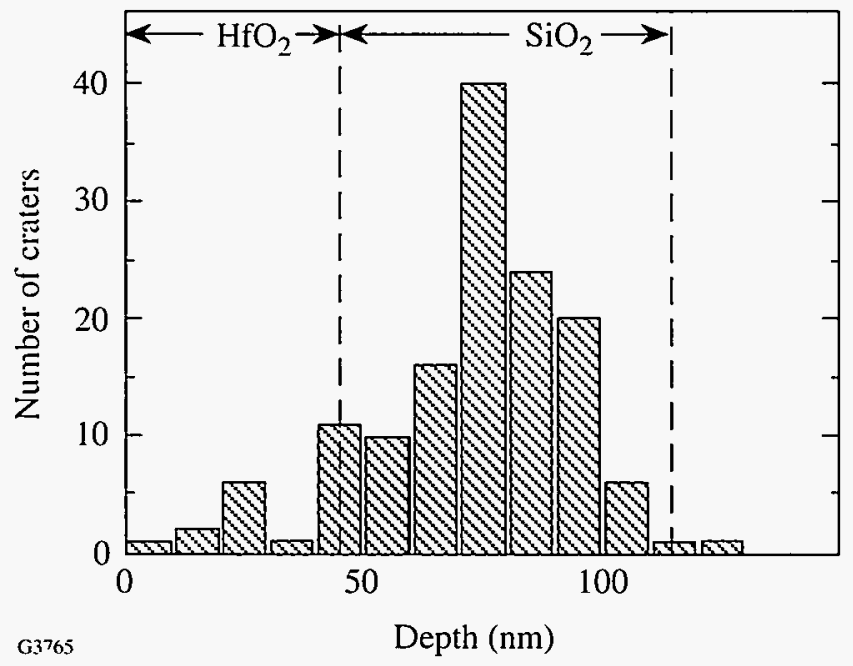

Figure 62.11

Crater-depth distribution at the central portion of a moderate-damage area ( $20 \%$ above threshold fluence) in the $\mathrm{HfO}_{2} / \mathrm{SiO}_{2}$ system. The distribution is now fully shifted into the $\mathrm{SiO}_{2}$ layer, indicative of the dominance of heatpropagation effects under the $20 \%$-above-threshold fluence conditions.

pate heat into the lower layer, extending the effective material modification to greater depths. This process is eased by the lower melting point of the second layer.

Figures 62.9 and 62.10 show also the $\mathrm{E}^{2}$-optical-intensity dependence inside the first two stack layers, which peak exactly at the interface. Other local maxima are located at the interface between the third and fourth layers, etc., with each deeper maximum dropping in value relative to the previous one. In good coatings, it is therefore not surprising to find that, at values near threshold, only the absorbers near the largest intensity maximum contribute to crater formation.

It has long been suspected that, either for manufacturing reasons or owing to intrinsic physics, the discontinuities associated with interfacial areas are absorber locations themselves and, as such, are drivers for laser damage. The lack of any clear evidence for crater depths peaking at interfaces (the apparent interface peak in Fig. 62.9 is not nearly strong enough not to disappear after a small bin-size adjustment) suggest that, for state-of-the-art coatings, interface issues have ceased to be damage-critical issues.

b. $\mathrm{Sc}_{2} \mathrm{O}_{3} / \mathrm{SiO}_{2}$. In the $\mathrm{Sc}_{2} \mathrm{O}_{3} / \mathrm{SiO}_{2}$ system the top $\mathrm{Sc}_{2} \mathrm{O}_{3}$ layer is only $25.4 \mathrm{~nm}$ thick, and the peak of the $\mathrm{E}^{2}$-intensity distribution has been designed to not coincide with the layer interface. For this system, the intensity peaks at a depth of $65 \mathrm{~nm}$. Both factors bias the crater-depth distribution toward the $\mathrm{SiO}_{2}$ layer, as seen in Figs. 62.12 and 62.13 for the cases of weak damage and peripheral moderate damage. As was true for $\mathrm{HfO}_{2} / \mathrm{SiO}_{2}$, no craters originate from beneath the second layer. However, the shift of the distribution peak in going from weak to moderate damage $\left(\sim 40 \mathrm{~nm}\right.$ in $\left.\mathrm{HfO}_{2} / \mathrm{SiO}_{2}\right)$ is more modest in this system. The 10 -nm difference between distribution peaks in Figs. 62.12 and 62.13 is too small to be considered significant in light of the $10-\mathrm{nm}$ bin width.

The trailing edges of the distributions shown in Figs. 62.12 and 62.13 drop faster with depth than the incident intensity,

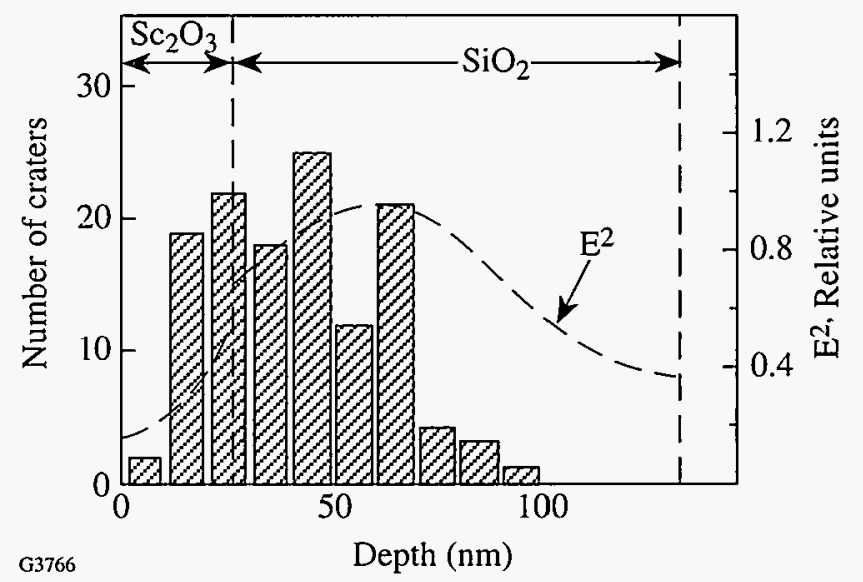

Figure 62.12

Crater-depth distribution (histogram) for weak damage in the $\mathrm{Sc}_{2} \mathrm{O}_{3} / \mathrm{SiO}_{2}$ system. Owing to the relatively thin (25.4-nm) $\mathrm{Sc}_{2} \mathrm{O}_{3}$ layer, the $E$-field, i.e., intensity, peaks inside the $\mathrm{SiO}_{2}$ layer. 


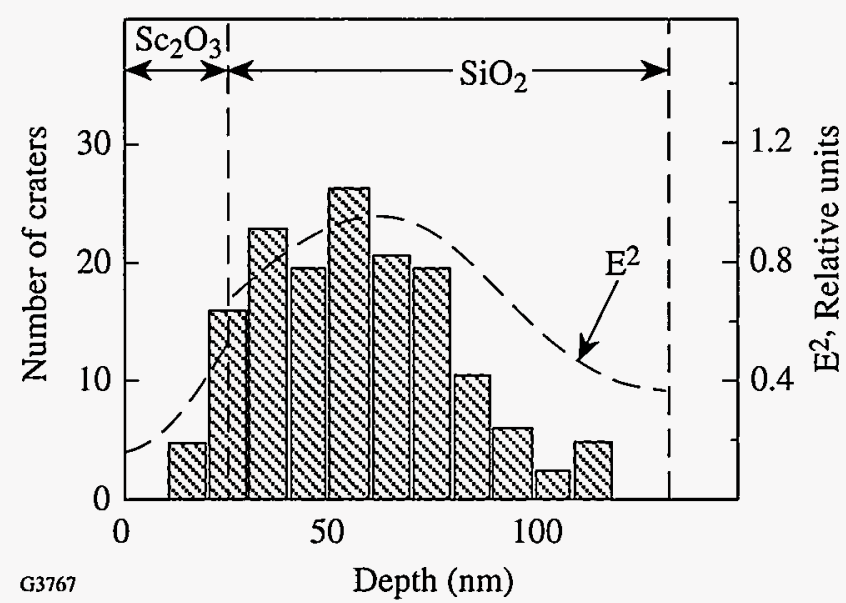

Figure 62.13

Crater-depth distribution (histogram) for moderate-damage (periphery) conditions in the $\mathrm{Sc}_{2} \mathrm{O}_{3} / \mathrm{SiO}_{2}$ system. The distribution is almost fully bracketed within the $\mathrm{SiO}_{2}$ layer.

which is not unexpected based on energy-balance grounds. If one simply assumes that for a conical crater to be formed the entire conical volume will be uniformly affected by heat, then the energy required for such heating will scale with the cone depth $h$ as, at most, $h^{3}$. In reality, this dependence is probably less severe. The distribution's trailing edge already scales nearly as $1 / h^{3}$, indicating that the exponent must be significantly smaller in light of the monotonously declining intensity dependence over the same depth interval.

\section{Conclusions}

Mapping of pulsed-laser-induced damage morphologies in UV high-reflector coatings by AFM has shown the dominant damage feature to be submicron-lateral-sized craters that appear to be independent of micron-scale film growth defects (nodules). The local number density of these defect craters follows the intensity profile over the full laser-beam profile (hundreds of microns) but appears random over scale lengths of a few microns. The $700^{\circ} \mathrm{K}$ to $1000^{\circ} \mathrm{K}$ difference in the melting point between the high- and low-index medium in these stacks results in peculiar crater formation in the $\mathrm{Sc}_{2} \mathrm{O}_{3} /$ $\mathrm{SiO}_{2}$ system, where crater-wall structural evidence shows melting and glass forming in the $\mathrm{SiO}_{2}$ layer, while the crater walls along the $\mathrm{Sc}_{2} \mathrm{O}_{3}$ layer maintain columnar structure. A preponderance of craters originating in the second $\left(\mathrm{SiO}_{2}\right)$ layer points toward heat transfer across the interface and lower-layer melting by absorbers located in the top layer as the primary cause. Together with the observation that the smallest measured craters allow for starting absorber sizes of $<10 \mathrm{~nm}$, this distribution points toward randomly distributed nano-cluster absorbers as the sources involved in the energy transfer from the optical field to the porous film medium. Whether these nano-clusters owe their absorptivity to unbound charges of metallic character or to charges localized in broken-stoichiometry states (point-defect states) remains to be resolved. The oxide deposition process certainly does not rule out bursts of impurity or non-stoichiometric events during which such nanoclusters could be dispersed into the coating stack. Similarly, at this time details of the damage kinetics are still speculative. The very low number density of nodules encountered leaves no doubt that few, if any, of these nano-clusters act as seeds for growth nodules, and that the nodules themselves may survive irradiation even though craters are formed within their immediate vicinity.

\section{ACKNOWLEDGMENT}

This work was supported by the U.S. Department of Energy Office of Inertial Confinement Fusion under Cooperative Agreement No. DE-FC0392SF19460, the University of Rochester, and the New York State Energy Research and Development Authority. The support of DOE does not constitute an endorsement by DOE of the views expressed in this article. This work was carried out on equipment of the Center for Optics Manufacturing (COM)-a DOD Center of Excellence by the U.S. Army Materiel Command.

\section{REFERENCES}

1. M. R. Kozlowski et al., in Scanning Microscopy Instrumentation, edited by G. S. Kino (SPIE, Bellingham, WA, 1991), Vol. 1556 pp. 68-78.

2. M. C. Staggs et al., in Laser-Induced Damage in Optical Materials: 1991, edited by H. E. Bennett et al. (SPIE, Bellingham, WA, 1992), Vol. 1624, pp. 375-385.

3. M. C. Staggs et al., in Laser-Induced Damage in Optical Materials: 1992, edited by H. E. Bennett et al. (SPIE, Bellingham, WA, 1993), Vol. 1848, pp. 234-242.

4. R. J. Tench, R. Chow, and M. R. Kozlowski, in Laser-Induced Damage in Optical Materials: 1993, edited by H. E. Bennett et al. (SPIE, Bellingham, WA, 1994), Vol. 2114, pp. 415-425.

5. K. L. Westra and D. J. Thomson, J. Vac. Sci. Technol. B 12, 3176 (1994).

6. S. Papernov and A. Schmid, "A Comparison of Laser-Induced Damage Morphology in Three Model Thin-Film Systems: $\mathrm{HfO}_{2}$, $\mathrm{Y}_{2} \mathrm{O}_{3}$, and $\mathrm{Ta}_{2} \mathrm{O}_{5}$," to be published in SPIE's Laser-Induced Damage in Optical Materials: 1994.

7. W. H. Lowdermilk and D. Milam, IEEE J. Quantum Electron. QE-17. 1888 (1981).

8. T. W. Walker, A. H. Guenther, and P. E. Nielsen, IEEE J. Quantum Electron. QE-17, 2041 (1981). 


\section{The OMEGA Laser Pulse-Shaping System}

Laser-fusion experiments require that high-energy, temporally shaped optical pulses be generated and applied to fusion targets. On the OMEGA laser, low-energy optical pulses will be shaped with amplitude modulators and injected into the laser system for amplification and delivery to the laser-fusion targets. A layout of the pulse-shaping system is shown in Fig. 62.14. The output of a single-mode Nd:YLF laser is sliced to produce a 10- to 15-ns square optical pulse. This pulse is sent through two integrated-optic amplitude modulators operated in series and fabricated on a single, fiber-coupled $\mathrm{LiNbO}_{3}$ waveguide. Shaped electrical pulses, synchronized with the input optical square pulse, are applied to the modulators. This shaped optical pulse from the modulators is then preamplified in a regenerative amplifier (regen) and sent to the OMEGA amplifier chains. The shaped electrical pulses are produced using optically activated $\mathrm{Si}$ photoconductive (PC) switches and variable impedance microstrip lines. Activation of the $\mathrm{Si}$ PC switches is achieved using an optical pulse that has been steepened by the stimulated Brillouin scattering process.

Given the desired optical pulse shape and energy on target, the required output optical pulse shape from the modulator is determined by modeling the temporal pulse distortions intro- duced by the OMEGA laser system from all sources and then using this model as a transfer function to relate the input to output pulse shapes from the system. This model, available in the form of a computer code (RAINBOW), takes into account such factors as the effects of the frequency-tripling process and gain saturation in the amplifiers. Since the optical transfer function of a modulator is well known in terms of the voltage waveform applied to the modulator, a knowledge of the optical pulse shape required on target uniquely determines the required voltage waveform that must be applied to the modulator.

\section{Optical Modulators}

A schematic of a typical dual-amplitude, fiber-coupled, waveguide integrated-optic modulator is shown in Fig. 62.15. The input and output fibers are single-mode polarizationpreserving fiber with industry-standard FC/APC connectors. Light launched into the input fiber is coupled into a waveguide in the $\mathrm{LiNbO}_{3}$ electro-optic crystal. A Y-branch is used to split the signal and form two arms of a Mach-Zehnder interferometer. Electrodes are placed around the interferometer arms so that a voltage applied to the RF port produces a phase shift in each arm of the interferometer. The polarity of these electrodes is arranged such that the device operates in a push-pull fashion,

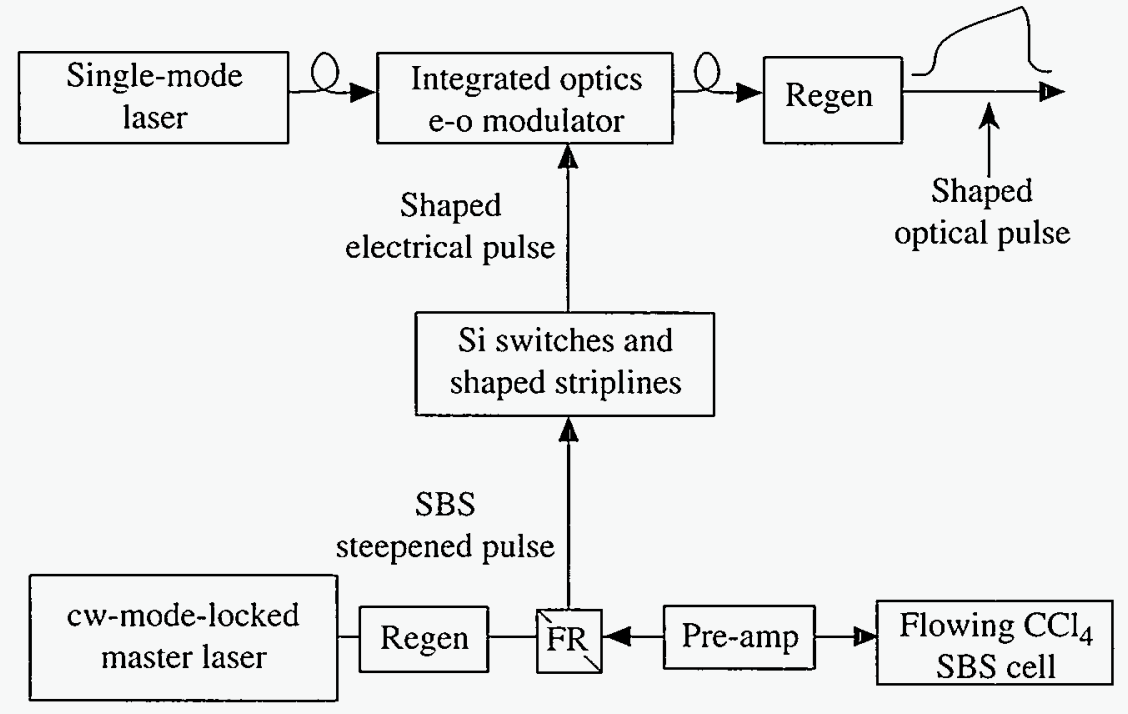

E7021
Figure 62.14

A block diagram of the OMEGA optical-pulse-shaping system. The electrical-pulse-generation portion of the system uses Si photoconductive switches that are activated with an SBS-steepened optical pulse. 


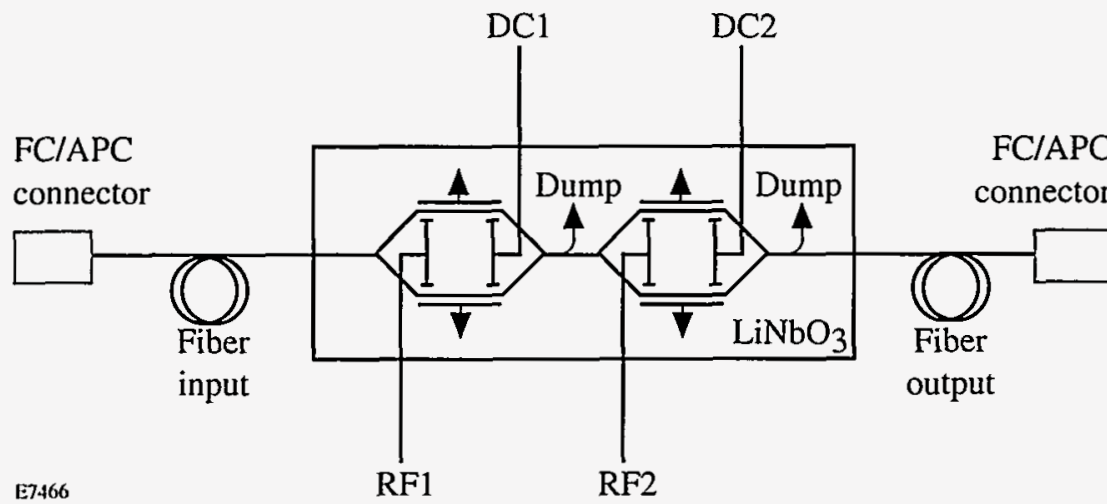

\author{
Figure 62.15 \\ The dual-amplitude, Mach-Zehnder, waveguide \\ integrated-optic modulator used for pulse shaping. \\ Shaped voltage waveforms are applied to the RF \\ input ports, and each modulator has a DC input port \\ for setting the dc offset.
}

i.e., the phase shift in the two arms is cumulative. A second Ybranch is used to coherently combine the two beams and send the shaped pulse to the next stage, while the unwanted radiation is sent to an appropriate beam dump. The device incorporates two identical modulators in series. The output optical pulse intensity from a modulator is given by

$$
I(t)=I_{0}(t) \cos ^{2}\left[\frac{\pi}{2} \frac{V(t)}{V_{\pi}}+\phi\right]
$$

where $I_{0}(t)$ is the input optical pulse intensity to the modulator, $V(t)$ is the applied voltage, $V_{\pi}$ is the half-wave voltage [the voltage required to drive the modulator from its maximum to its minimum transmission $(<10 \mathrm{~V})]$, and $\phi$ is an overall phase that can be controlled by an applied $0-$ to $10-\mathrm{V}$ dc bias (provided by a computer with an A/D board). In our case, the input optical pulse to the modulator is a square optical pulse ( 10-ns FWHM) obtained by "slicing out" the center of a single-longitudinal-mode Nd:YLF laser pulse (pulsewidth -100-ns FWHM) using conventional Pockels cells. The modulators are designed for 1054-nm-wavelength operation and have traveling wave electrodes to provide an $8-\mathrm{GHz}$ bandwidth.

\section{Electrical Waveform Generation}

To produce shaped optical pulses from the modulators, temporally shaped voltage waveforms [ $V(t)$ in Eq. (1)] must be applied to the modulators. (The modulator is dc biased so that $\phi=\pi / 2$.) The electrical-pulse-generation system that shapes the voltage waveforms applied to the modulators is modeled after a design developed at Lawrence Livermore National Laboratory (LLNL). ${ }^{1}$ The system consists of two Si PC switches, a microstrip charge line, and a variable impedance microstrip line as shown in Fig. 62.16. The microstrip charge line is placed between two PC switches and is charged to approximately $80 \mathrm{~V}$. The opposite side of one PC switch is connected to the modulator, while the opposite side of the other PC switch is connected to the terminated variable-impedance microstrip line. When the PC switch near the variable-impedance microstrip line is activated (closed), a square pulse is sent to the

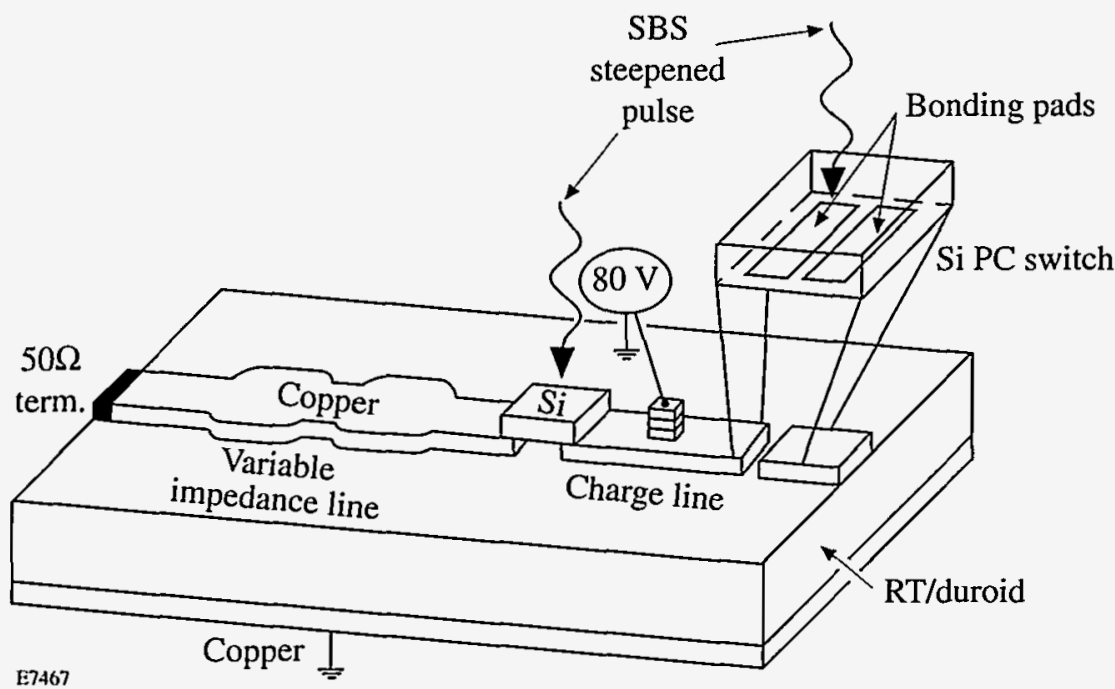

Figure 62.16

A detailed view of the electrical-pulse-generation system consisting of two optically activated Si PC switches, a microstrip charge line, and a terminated variable-impedance microstrip line. 
variable-impedance microstrip line. A voltage waveform is then reflected from the variable-impedance line with a specific shape depending on the impedance mismatches along the length of the line. This shaped voltage waveform propagates back through the PC switch and charge line toward the modulator. Just before the waveform reaches the modulator PC switch. this switch is activated, allowing the voltage waveform to be applied to the RF input port of the modulator. The exact shape of the voltage waveform can be controlled by the judicious placement of impedance variations along the length of the variable-impedance microstrip line.

The system of microstrip lines and switches is designed to be impedance matched to the $50-\Omega$ input impedance of the modulators and test equipment used. Microstrip lines were chosen instead of strip lines to minimize the impedance mismatch at the Si switches. The charge line and variable-impedance microstrip lines are fabricated in $0.79-\mathrm{mm}$-thick RT/duroid 5880 microwave laminate having $36-\mu \mathrm{m}$-thick $\mathrm{Cu}$ on both sides. An impedance variation along the line is obtained by adjusting the width of the top Cu electrode. The exact geometry is easily calculated knowing the required voltage waveform that must be produced. The reflection coefficient along the line can be calculated using a layer-peeling technique. ${ }^{2}$ The impedance as a function of position along the line is then obtained from the reflection coefficient along the line. Finally the width of the electrode along the line is calculated using simple relationships between the material parameters and the impedance variations. ${ }^{3}$ To fabricate the microstrip line, the $\mathrm{Cu}$ material on one side is machined with a precision programmable milling machine to produce the desired width as a function of length. The charge line is a constant $50-\Omega$ microstrip line ( $\mathrm{Cu}$ width $=2.38 \mathrm{~mm}$ ) with a $2-\mathrm{mm}$ gap at both ends to facilitate Si switch mounting. The Si switches, $2 \mathrm{~mm}$ long by $2.38 \mathrm{~mm}$ wide (to match the width of the $50-\Omega$ charge line) by $0.5 \mathrm{~mm}$ thick, have bonding pads with $1-\mathrm{mm}$ separation. The switches are low temperature soldered across each gap in the charge line with the bonding pads facing the $\mathrm{Cu}$ of the microstrip line, as shown in Fig. 62.16. The switches are illuminated from the back (opposite side from the contact pads) using $1054-\mathrm{nm}$ laser radiation with $\sim 0.5 \mathrm{~mJ}$ of energy per pulse. The laser radiation is absorbed throughout the entire volume of the 0.5 -mm-thick Si switch, which results in switch activation. When illuminated with a laser pulse, the switch resistance changes from its dark value of approximately $600 \mathrm{k} \Omega$ to its activated resistance of less than $1 \Omega$ as the absorbed photons generate electron hole pairs in the $\mathrm{Si}$ and reduce the resistivity of the material.
The square pulse sent to the variable-impedance microstrip line must contain frequency components high enough to reflect the pulse shapes of interest. Photoconductive switches can be activated (closed) in a time comparable to the rise time of the activating optical pulse $(<100 \mathrm{ps})$, which in this case is a pulse that has its leading edge steepened by the stimulated Brillouin scattering (SBS) process. A typical square pulse produced by activating one switch only is shown in Fig. 62.17. The rising edge of the electrical pulse is approximately $80 \mathrm{ps}$, as measured with a Tektronix 7250 oscilloscope ( $10-\mathrm{GHz}$ bandwidth).

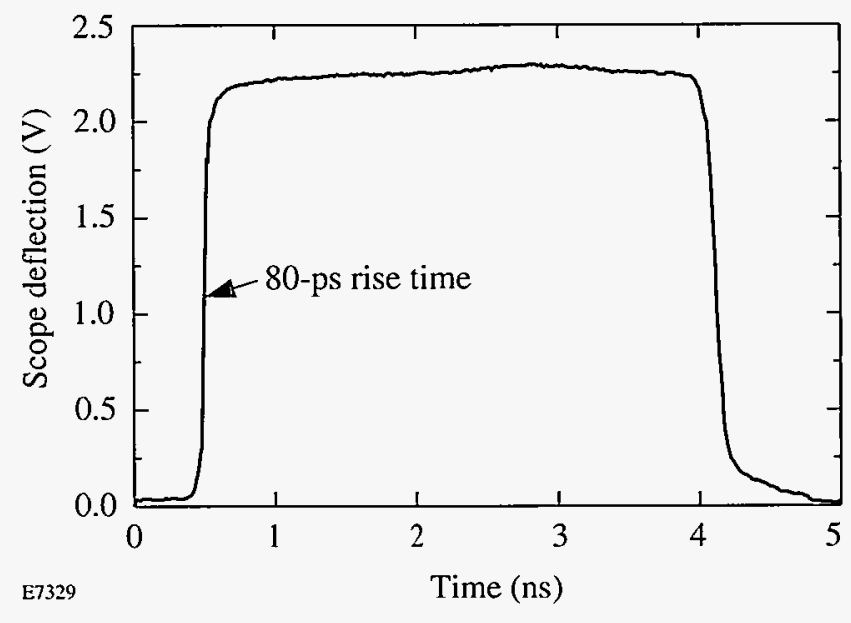

Figure 62.17

A square electrical pulse generated by the charge line and a Si PC switch. The pulse has a rise time of approximately 80 ps.

High bandwidth must be maintained throughout the entire system. High-bandwidth SMA end launchers and cables are used to transfer the electrical pulses from the microstrip line to the modulator or test equipment being used. Care is taken to minimize the impedance mismatch in the charge line at the point where the charging voltage is applied; this is done by charging through three board-mounted chip resistors $(\sim 10 \mathrm{k} \Omega$ each) stacked in series, as shown in Fig. 62.16. Less than 0.6\% reflection at $10 \mathrm{GHz}$ is produced by this process, as determined from time-domain reflectometer (TDR) measurements. A TDR of the complete microstrip line can be taken by soldering copper strips across the gaps in place of the $\mathrm{Si}$ switches. Figure 62.18 shows (1) the TDR measurement of a variable impedance microstrip line (taken with a $20-\mathrm{GHz}$-bandwidth HP 5420B TDR oscilloscope) with $\mathrm{Si}$ switches removed, compared to the design shape, and (2) the shaped electrical pulse transferred into the Tektronix 7250 oscilloscope after passing through the two Si switches that have been activated as described above. From Fig. 62.18 we see that, although the 
present $\mathrm{Si}$ switches clearly degrade the inherent frequency response of the system, high-bandwidth shaped electrical pulses can ultimately be achieved with this pulse-generating system. Research is continuing in this area to improve the overall system performance.

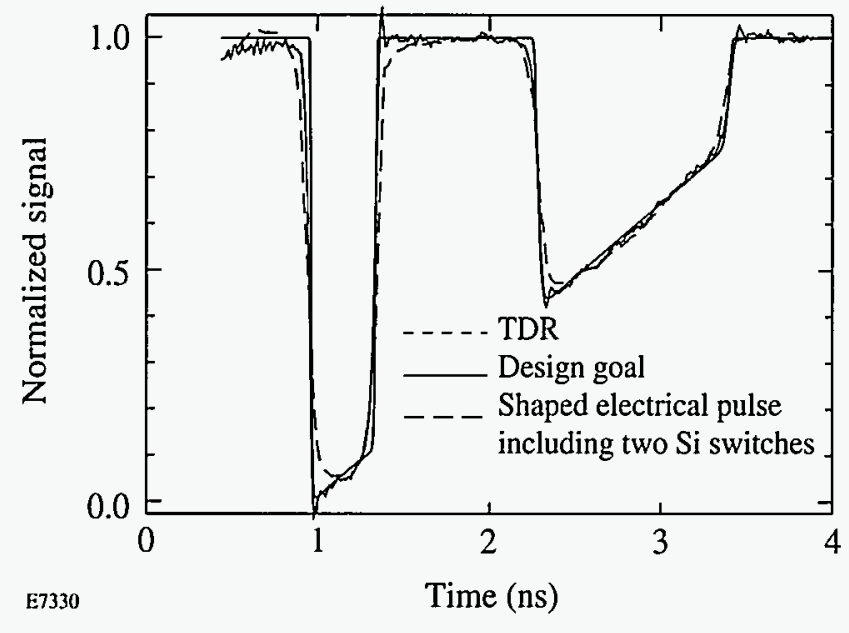

Figure 62.18

Time-domain reflectometer (TDR) measurement of a variable impedance microstrip line (dotted line) compared to the design goal (solid line). The dashed line is the measured shaped electrical pulse after propagation through two Si PC switches.

\section{PC Switch Activation}

To produce fast closing times the PC switches must be activated by illuminating them with optical energy in as short a time as possible. Once the switches are closed, they must stay closed for a long time in comparison to the desired electrical pulse shapes of interest ( 10 ns). Silicon PC switches were chosen for this application because of their long recombination times ( 100 to $200 \mu \mathrm{s})$. The switches are activated with fast-risetime, high-contrast optical pulses generated by the SBS process. ${ }^{4-5}$ The experimental setup is shown in Fig. 62.14.

A 1054-nm, 1- to 3-ns FWHM pulse (controlled by intracavity etalons ${ }^{6}$ ) from a Nd:YLF regen is amplified to 3 to $5 \mathrm{~mJ}$ and focused into a liquid cell containing carbon tetrachloride $\left(\mathrm{CCl}_{4}\right)$. A Brillouin-Stokes pulse is generated in the backward direction from this cell due to the SBS process. The SBS process is an intensity-dependent, nonlinear process where the incident laser Bragg-scatters in the backward direction from a driven acoustic wave in the medium. The process can be modeled as a pure gain process whereby a backward-traveling Brillouin noise signal experiences exponential-type gain throughout the focal region of the laser and grows into the
Brillouin-Stokes signal. The gain coefficient depends on the laser intensity and the response time of the Brillouin medium (less than $1 \mathrm{~ns}$ for $\left.\mathrm{CCl}_{4}\right) .{ }^{7}$ The low intensity at the beginning of the laser pulse is transmitted through the cell, with little energy converted to the Brillouin signal. As the laser intensity in the cell increases during the laser pulse, the cell begins to generate Brillouin Stokes energy very rapidly due to the nonlinear buildup of the acoustic wave. The process quickly saturates, after which the laser scatters into the Stokes wave with high efficiency. The backscattered Brillouin pulse from the SBS cell has a fast-rising edge ( $<100$-ps measurement limited) due to this Brillouin pulse steepening, while the back of the pulse approximates that of the laser. The reflected Brillouin pulse is sent back through the preamplifier and switched out using polarization optics and a Faraday rotator (FR). The SBS pulse is then split and used to activate both PC switches by introducing an appropriate timing delay between the two pulses.

Typical SBS reflected pulses for different incident laser energies are shown in Fig. 62.19. As the incident laser energy is varied, the location in time of the fast-rising edge of the SBS pulse changes. As seen in Fig. 62.19, as we increase (decrease) the laser energy, the SBS process turns on earlier (later). Hence, amplitude fluctuations in the laser may result in timing jitter of the electrical-pulse-generation system. To obtain electrical waveforms from the pulse-generation system that are

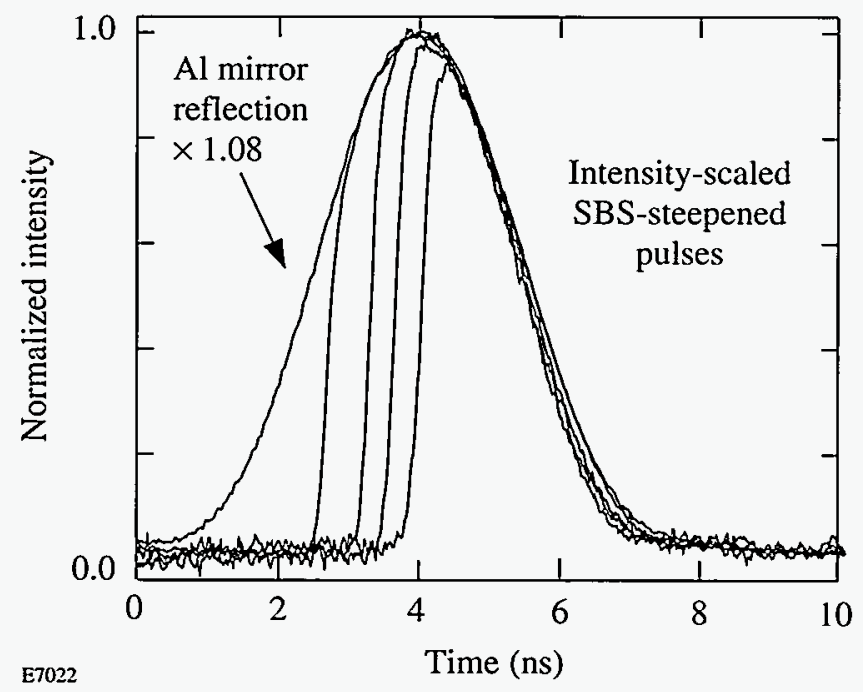

Figure 62.19

Fast-rise-time optical pulses produced by SBS pulse steepening with varying laser input energy. As the incident laser energy is increased (decreased), the SBS pulse turns on earlier (later). The reflected SBS pulse has a rise time of less than $100 \mathrm{ps}$. 
accurately synchronized to a master timing reference, the laser that activates the PC switches must be amplitude stable. The laser amplitude in our setup is actively stabilized to within $3 \%$ using an optical feedback system that controls the cavity losses during the pulse build-up phase. A shaped optical pulse from the pulse-shaping system (including the Si PC switches, variable-impedance microstrip line, optical modulator, and regen preamplifier) was produced, and the shot-to-shot timing jitter between the shaped optical pulse and the master cw-modelocked laser pulse injected into the regen that activates the PC switches was measured. An absolute timing jitter of less than 30 ps was measured using the Tektronix 7250 oscilloscope ( $\sim 10-\mathrm{GHz}$ bandwidth) and fast detectors ( 3.5-GHz bandwidth).

The SBS process has an additional advantage when using $\mathrm{Si}$ PC switches. The SBS process is nonlinear, and for low incident laser energy into the SBS cell, essentially no Brillouin energy is reflected; hence, all laser prepulse noise is eliminated, resulting in a very-high on/off contrast ratio. This is important for $\mathrm{Si}$ switch applications because the recombination times are long ( 100 to $200 \mu \mathrm{s}$ ) and the switch will integrate the incident energy during this recombination time. Elimination of all prepulse optical noise ensures proper switch performance.

\section{System Performance}

Optical pulses have been produced with the pulse-shaping system described above. Figure 62.20 shows an optical pulse shape produced by the system (solid line). To produce this shape, a square optical pulse ( 10-ns FWHM) is used as input to the modulator. This pulse is obtained by slicing out the center of a single-longitudinal-mode Nd:YLF laser pulse (pulse width 100-ns FWHM) using conventional Pockels cells, as was mentioned previously. The square pulse is then transferred to the modulator through optical fibers. Synchronized with this optical pulse is the electrical pulse (Fig. 62.18) that was produced by the electrical pulse generator described above. Figure 62.20 also shows the optical pulse shape expected from the modulator (dashed line) if the design goal of Fig. 62.18 is substituted into the modulator transfer function [Eq. (1)], and the optical pulse shape expected from the modulator (dotted line) if the measured shaped electrical pulse of Fig. 62.18 is substituted into the modulator transfer function [Eq. (1)].

The optical pulse in Fig. 62.20 was measured at the output of the modulator with a $25-\mathrm{GHz}$ bandwidth detector (New Focus 1414). The energy at this point in the pulse-shaping system is approximately $10 \mathrm{~nJ}$ per pulse and is too low to make temporal measurements of a single pulse. The measurement

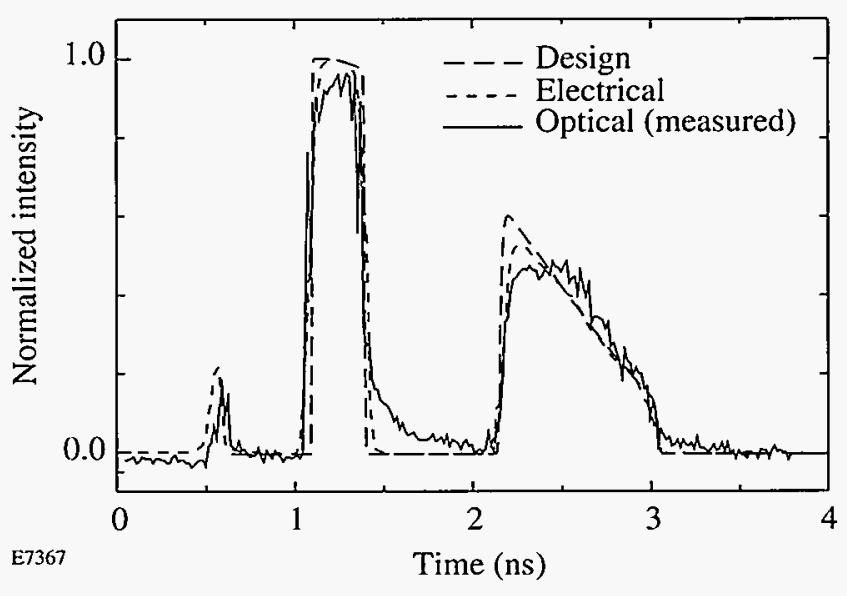

Figure 62.20

A comparison of expected and observed optical pulse shapes from the OMEGA laser's pulse-shaping system. Actual measured optical pulse shape from the modulator (solid line); optical pulse shape expected from the modulator if the design goal of Fig. 62.18 is substituted into the modulator transfer function [Eq. (1)] (dashed line); optical pulse shape expected from the modulator if the measured shaped electrical pulse of Fig. 62.18 is substituted into the modulator transfer function [Eq. (1)] (dotted line).

was taken with a high-bandwidth sampling oscilloscope (HP 5420B) and includes averaging over many pulses. The optical pulse shape in Fig. 62.20 appears to deviate from the expected pulse shape. We believe that the deviations are largely due to the detector response. In fact, our measurements show that large discrepancies occur between high-bandwidth detectors when measuring the same optical pulse. These detectors are designed to measure short pulses with fast rise times but have varying responses when trying to measure long pulses with fast rising structure. Work is continuing in this area to make accurate measurements of these shaped pulses. A streak camera will be used to measure these pulses and is expected to provide more accurate results.

In summary, an optical-pulse-shaping system has been designed that is capable of meeting the future pulse-shaping requirements for the OMEGA laser. The system has been tested and is capable of producing shaped optical pulses with 50 - to 100-ps structure over a several-nanosecond pulse envelope.

\section{ACKNOWLEDGMENT}

This work was supported by the U.S. Department of Energy Office of Inertial Confinement Fusion under Cooperative Agreement No. DE-FC0392SF1 9460, the University of Rochester, and the support of the Frank Horton Graduate Fellowship Program. The support of DOE does not constitute an endorsement by DOE of the views expressed in this article. 


\section{REFERENCES}

1. R. B. Wilcox et al., in Laser Coherence Control: Technology and Applications, edited by H. T. Powell and T. J. Kessler (SPIE, Bellingham, WA, 1993), Vol. 1870, pp. 53-63.

2. S. C. Burkhart and R. B. Wilcox, IEEE Trans. Microw. Theory Tech. 38, 1514 (1990).

3. B. C. Wadell, Transmission Line Design Handbook (Artech House, Boston, 1991).
4. T. de Saxce and P. Picart, in Optically Activated Switching III, edited by R. A. Falk (SPIE, Bellingham, WA, 1993), Vol. 1873, pp. 262-271.

5. A. Okishev, M. D. Skeldon, and W. Seka, in CLEO '94, 1994 Technical Digest Series, Volume 8, Anaheim, CA, 8-13 May 1994, paper CTuQ5.

6. M. D. Skeldon and S. T. Bui, J. Opt. Soc. Am. B 10, 677 (1993).

7. W. Kaiser and M. Maier, in Laser Handbook, edited by F. T. Arecchi and E. O. Schulz-Dubois (North-Holland, Amsterdam, 1972), Vol. 2 , p. 1077. 


\section{Direct-Drive Irradiation Uniformity for the NIF}

The National Ignition Facility (NIF) can have the flexibility to perform direct-drive experiments, in addition to indirect drive, if two key elements are included in its design: (1) The facility must be able to redirect 24 of the beam clusters to new beam ports near the "equator" of the target chamber, and (2) twodimensional smoothing by spectral dispersion ${ }^{1}$ (2-D SSD) must be implemented.

In this article, we first address how the indirect-drive irradiation geometry must be modified to accommodate direct drive. Emphasis is placed on finding an acceptable direct-drive geometry that minimizes the amount of reconfiguration. Tolerances for energy imbalance, beam mispointing, and target-positioning errors are discussed for the proposed irradiation geometry. The beam uniformity that can be achieved using 2-D SSD is also examined. One related laser design issue that affects the level of uniformity is pinhole size. The pinholes determine the maximum amount of angular spectral dispersion that can be used with SSD. The dependence of uniformity on pinhole size is discussed. Finally, the SSD-smoothed beam profile is combined with the direct-drive beam geometry to calculate the irradiation uniformity on a spherical target with multiple overlapping beams.

\section{Beam Placement}

Because the NIF will have only a single target chamber (designed primarily for indirect drive), a direct-drive component must be integrated into the system without major modifications. The current design for beam placement at the NIF target chamber, given in Table 62.I, has 48 beams in eight latitudinal rings clustered near the poles. Each beam consists of a cluster of four beamlets, separated in wavelength by about $5 \AA$. Such an irradiation scheme, taken as a whole, does not lend itself to direct drive. However, a portion of the beam positions would be suitable if additional beam ports were added near the equator. Such configurations have been considered by Mark, ${ }^{2}$ who pointed out that direct-drive beams need not be uniformly disposed but could be placed in rings at polar angles given by the zeros of a Legendre polynomial. However, in general, the beams from different rings must have different energies, corresponding to the weights of Gaussian quadrature. One such configuration, which closely meshes with the current NIF design, is based on rings of beams at the zeros of the $\mathrm{P}_{6}$ Legendre polynomial, with polar angles given by $\theta=21.17^{\circ}$, $48.60^{\circ}, 76.19^{\circ}, 103.81^{\circ}, 131.40^{\circ}$, and $158.83^{\circ}$. Examination of the beam-port positions in Table 62.I shows that four of the rings in that design correspond very well to four of these angles, namely the ports at $23.49^{\circ}, 48.27^{\circ}, 131.73^{\circ}$, and $156.73^{\circ}$. For half of the beam ports, no modification would be required (except possibly for a small pointing correction so that the beam axes pass through the target surface, closer to the required latitudes). The remaining 24 beam clusters would have to be redirected into new beam ports positioned at $76.19^{\circ}$ and $103.80^{\circ}$ (Table 62.II).

The number of beam clusters in each ring for this directdrive configuration is 4,8 , and 12 for the upper hemisphere, and similarly for the lower. The laser energies coming from each of these rings must be in the proportion of 1 to 2.105 to 2.731. Distributing this energy among the beams in each ring, we find that the relative energies for beams from different rings are $0.95,1.00$, and 0.86 , which implies that beams from the polar and equatorial rings must be reduced in energy relative to beams from the ring at $\theta=48^{\circ}$. The net result is that $\sim 8 \%$ of the available laser energy cannot be used for target irradiation, which represents a relatively small energy penalty for the flexibility of adding a direct-drive option to the current NIF design.

The irradiation uniformity for this 48 -cluster configuration is shown in Fig. 62.21. The rms nonuniformity is plotted as a function of how much the beam overfills the target, for a smooth eighth-order supergaussian beam profile. (The edge of the beam was defined as the $5 \%$ intensity contour.) The nonuniformity is entirely long-wavelength structure, characterized by how well the 48 overlapping clusters of beams can cover a sphere. (The effect of short-wavelength nonuniformity from structure on the individual beams is examined in the next section.) For conditions at the onset of irradiation, the beamto-target ratio should be chosen to be about 1.1. As the target implodes, this ratio becomes larger, and the long-wavelength 
Table 62.I: NIF beamport assignments — indirect drive

\begin{tabular}{|c|c|c|c|c|c|}
\hline Port \# & $\theta$ & $\phi$ & Port \# & $\theta$ & $\phi$ \\
\hline n01 & 23.4895 & 78.750 & $\mathrm{n} 25$ & 123.3311 & 5.625 \\
\hline n02 & 23.4895 & 168.750 & $\mathrm{n} 26$ & 123.3311 & 50.625 \\
\hline n03 & 23.4895 & 258.750 & n27 & 123.3311 & 95.625 \\
\hline n04 & 23.4895 & 348.750 & $\mathrm{n} 28$ & 123.3311 & 140.625 \\
\hline n05 & 31.9844 & 33.750 & n29 & 123.3311 & 185.625 \\
\hline n06 & 31.9844 & 123.750 & $\mathrm{n} 30$ & 123.3311 & 230.625 \\
\hline n07 & 31.9844 & 213.750 & n31 & 123.3311 & 275.625 \\
\hline n08 & 31.9844 & 303.750 & n32 & 123.3311 & 320.625 \\
\hline n09 & 48.2682 & 16.875 & n33 & 131.7317 & 28.125 \\
\hline n 10 & 48.2682 & 61.875 & n34 & 131.7317 & 73.125 \\
\hline $\mathrm{n} 11$ & 48.2682 & 106.875 & n35 & 131.7317 & 118.125 \\
\hline $\mathrm{n} 12$ & 48.2682 & 151.875 & n36 & 131.7317 & 163.125 \\
\hline $\mathrm{n} 13$ & 48.2682 & 196.875 & n37 & 131.7317 & 208.125 \\
\hline $\mathrm{n} 14$ & 48.2682 & 241.875 & n38 & 131.7317 & 253.125 \\
\hline $\mathrm{n} 15$ & 48.2682 & 286.875 & n39 & 131.7317 & 298.125 \\
\hline $\mathrm{n} 16$ & 48.2682 & 331.875 & $\mathrm{n} 40$ & 131.7317 & 343.125 \\
\hline $\mathrm{n} 17$ & 56.6688 & 39.375 & n41 & 148.1055 & 56.250 \\
\hline $\mathrm{n} 18$ & 56.6688 & 84.375 & n42 & 148.1055 & 146.250 \\
\hline $\mathrm{n} 19$ & 56.6688 & 129.375 & $\mathrm{n} 43$ & 148.1055 & 236.250 \\
\hline n20 & 56.6688 & 174.375 & $\mathrm{n} 44$ & 148.1055 & 326.250 \\
\hline $\mathrm{n} 21$ & 56.6688 & 219.375 & $\mathrm{n} 45$ & 156.7317 & 11.250 \\
\hline $\mathrm{n} 22$ & 56.6688 & 264.375 & n46 & 156.7317 & 101.250 \\
\hline $\mathrm{n} 23$ & 56.6688 & 309.375 & $\mathrm{n} 47$ & 156.7317 & 191.250 \\
\hline $\mathrm{n} 24$ & 56.6688 & 354.375 & $\mathrm{n} 48$ & 156.7317 & 281.250 \\
\hline
\end{tabular}

nonuniformity degrades slightly. The solid line in Fig. 62.21 is the result of pointing the polar ring of beams to the correct latitude on the target surface; the dashed line is without this correction. The effect of the beam-pointing correction is to reduce this contribution to nonuniformity by $30 \%$ to $50 \%$.

The effect on uniformity produced by energy imbalance among the beams is shown in Fig. 62.22. (The energy fluctuations among beams within a cluster were assumed uncorrelated.) Conditions with the same rms variation in energy can produce a relatively large spread in nonuniformity, as indicated by the bars in the figure, but it is all very-long-wavelength structure, corresponding to spherical harmonic modes 1 through 4 . Similar results were obtained for cluster pointing errors (Fig. 62.23). Target positioning errors, shown in Fig. 62.24, predominantly affect only the mode $\ell=1$. Generally, the combined contributions from these factors will add harmonically. These factors will make less than a $1 \%$ contribution to nonuniformity if the energy imbalance is less than $\sim 5 \%$, cluster pointing is better than $5 \%$ of the target radius, and target positioning errors are less than about $2 \%$ of the target radius.

The $8 \%$ reduction in laser energy can be avoided if the beamports for indirect drive can be shifted by a few degrees 
Table 62.II: NIF beamport assignments - direct drive

\begin{tabular}{|c|c|c|c|c|c|}
\hline Port \# & $\theta$ & $\phi$ & Port \# & $\theta$ & $\phi$ \\
\hline n01 & 23.4895 & 78.750 & $\mathrm{n} 25$ & 103.8000 & 15.000 \\
\hline n02 & 23.4895 & 168.750 & n26 & 103.8000 & 45.000 \\
\hline n03 & 23.4895 & 258.750 & n27 & 103.8000 & 75.000 \\
\hline n04 & 23.4895 & 348.750 & $\mathrm{n} 28$ & 103.8000 & 105.000 \\
\hline n05 & 48.2682 & 16.875 & n29 & 103.8000 & 135.000 \\
\hline n06 & 48.2682 & 61.875 & $\mathrm{n} 30$ & 103.8000 & 165.000 \\
\hline n07 & 48.2682 & 106.875 & n31 & 103.8000 & 195.000 \\
\hline n08 & 48.2682 & 151.875 & $\mathrm{n} 32$ & 103.8000 & 225.000 \\
\hline n09 & 48.2682 & 196.875 & $\mathrm{n} 33$ & 103.8000 & 255.000 \\
\hline $\mathrm{n} 10$ & 48.2682 & 241.875 & $\mathrm{n} 34$ & 103.8000 & 285.000 \\
\hline $\mathrm{n} 11$ & 48.2682 & 286.875 & $\mathrm{n} 35$ & 103.8000 & 315.000 \\
\hline $\mathrm{n} 12$ & 48.2682 & 331.875 & $\mathrm{n} 36$ & 103.8000 & 345.000 \\
\hline $\mathrm{n} 13$ & 76.1900 & 0.000 & n37 & 131.7317 & 28.125 \\
\hline $\mathrm{n} 14$ & 76.1900 & 30.000 & n38 & 131.7317 & 73.125 \\
\hline $\mathrm{n} 15$ & 76.1900 & 60.000 & n39 & 131.7317 & 118.125 \\
\hline $\mathrm{n} 16$ & 76.1900 & 90.000 & $\mathrm{n} 40$ & 131.7317 & 163.125 \\
\hline $\mathrm{n} 17$ & 76.1900 & 120.000 & $\mathrm{n} 41$ & 131.7317 & 208.125 \\
\hline $\mathrm{n} 18$ & 76.1900 & 150.000 & $\mathrm{n} 42$ & 131.7317 & 253.125 \\
\hline n19 & 76.1900 & 180.000 & $n 43$ & 131.7317 & 298.125 \\
\hline $\mathrm{n} 20$ & 76.1900 & 210.000 & $\mathrm{n} 44$ & 131.7317 & 343.125 \\
\hline $\mathrm{n} 21$ & 76.1900 & 240.000 & $\mathrm{n} 45$ & 156.7317 & 11.250 \\
\hline $\mathrm{n} 22$ & 76.1900 & 270.000 & $\mathrm{n} 46$ & 156.7317 & 101.250 \\
\hline$n 23$ & 76.1900 & 300.000 & $\mathrm{n} 47$ & 156.7317 & 191.250 \\
\hline $\mathrm{n} 24$ & 76.1900 & 330.000 & $\mathrm{n} 48$ & 156.7317 & 281.250 \\
\hline
\end{tabular}

without affecting indirect-drive target performance. One possibility ${ }^{3}$ is to move the rings at $23.49^{\circ}$ and $48.27^{\circ}$ to new positions at $21.00^{\circ}$ and $46.50^{\circ}$. The additional ring of 12 beams, in the upper hemisphere, would be placed at $75.75^{\circ}$ rather than $76.19^{\circ}$. The rings in the lower hemisphere would be at $104.25^{\circ}, 133.50^{\circ}$, and $159.00^{\circ}$. The direct-drive uniformity for this configuration is similar to the solid curve in Fig. 62.21, but with all beam axes now passing through the center of the target. The uniformity requirement for direct drive can be met by either configuration. Clearly, options requiring no energy reduction are preferred.

\section{Beam Smoothing by Spectral Dispersion (SSD)}

This section examines issues related to beam smoothing by spectral dispersion (SSD). The starting point for the calculations presented here is the SSD configuration proposed for the NIF by D. Eimerl ${ }^{4}$ during the ICFAC meeting at the University of Rochester on 18 May 1994. A key design issue for the NIF that affects optimization of SSD is the angular acceptance of the pinholes, which determines the amount of spectral dispersion that can be used by SSD. It will be shown below that if twice the dispersion specified by Eimerl is imposed (in just one dimension), then a substantial improvement in uniformity can be achieved. 


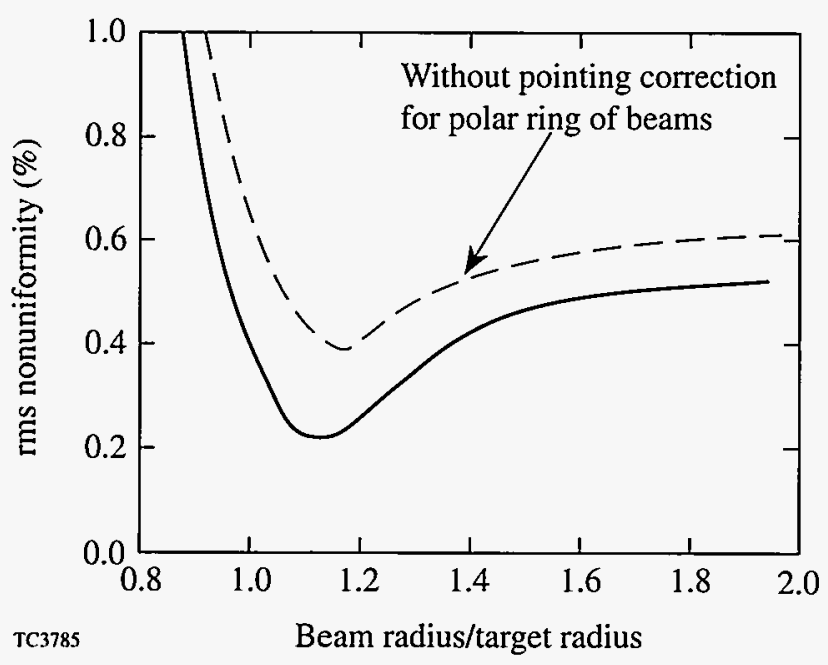

Figure 62.21

The rms nonuniformity for the 48-beam configuration as a function of the ratio of beam size to target size. An eighth-order supergaussian beam profile was used. (The effect of structure on the beams is shown in Fig. 62.29.) The solid line is the result of pointing the polar ring of beams to the optimal position on the target surface. The dashed line is without this correction.

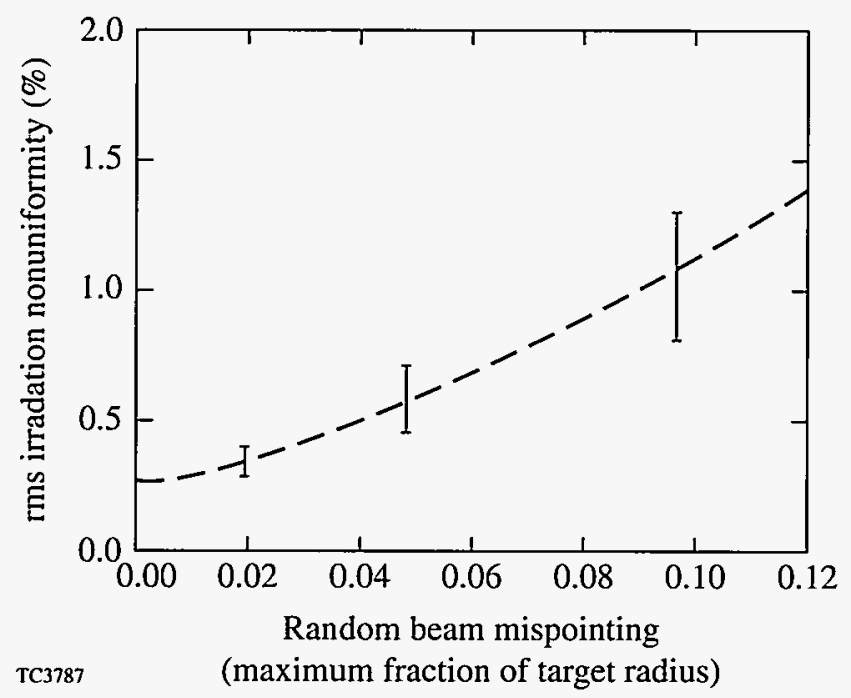

Figure 62.23

The rms irradiation nonuniformity as a function of cluster pointing error. All beams within a cluster were assumed to be pointed in the same direction.

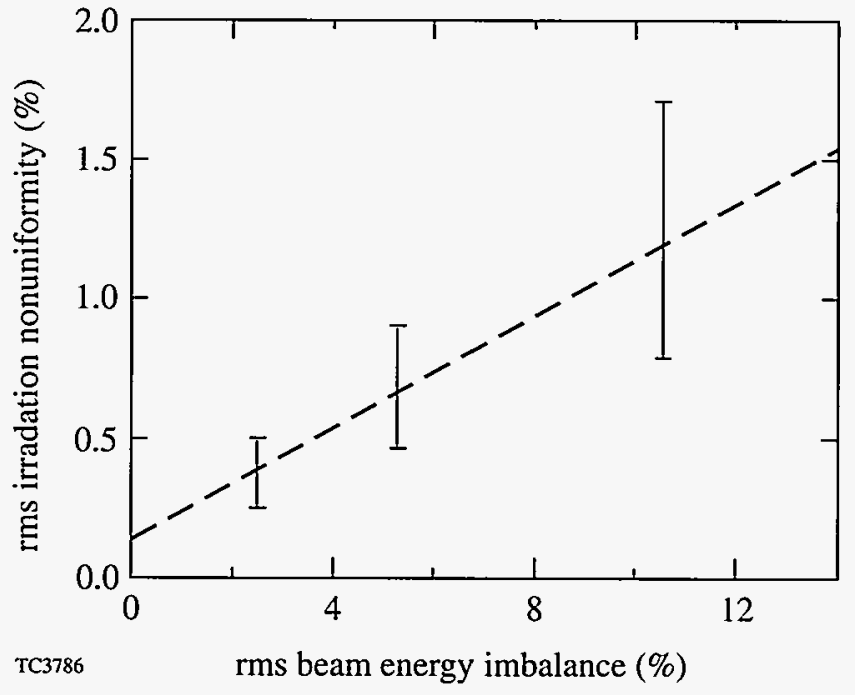

Figure 62.22

The rms irradiation nonuniformity as a function of the rms energy imbalance among the beams. The bars show the spread in irradiation nonuniformity that can occur for a given energy imbalance. Energy fluctuations among beams in a cluster were assumed to be uncorrelated.

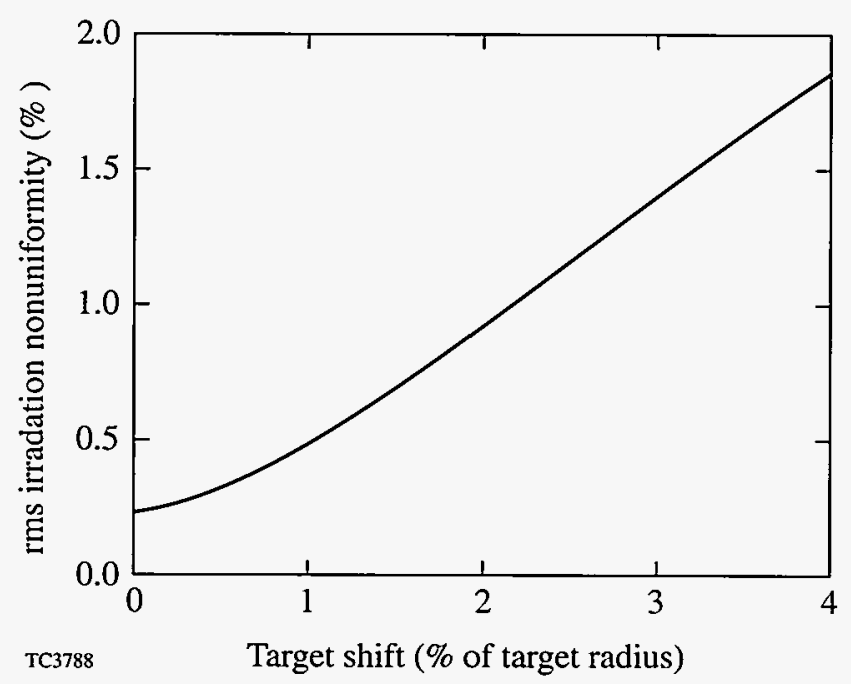

Figure 62.24

The rms irradiation nonuniformity as a function of target positioning error. 
The three sets of parameters that determine the single-beam uniformity achievable by SSD are those related to the phase plate, the polarization rotator (or shifter), and the spectrally dispersed bandwidth. The first two parameter sets will be mentioned only briefly as any adjustable parameters associated with them will affect mainly long-wavelength nonuniformities. The third parameter set will be discussed in more detail since this provides the primary smoothing mechanism for short-wavelength structure in the beam and a large number of options are available.

\section{Phase Plate}

The calculations presented here use a binary phase plate with an array of $250 \times 250$ square elements, which produces a $\operatorname{sinc}^{2}$ intensity envelope in the target plane. Superimposed upon this envelope is highly modulated speckle from the interference between rays from different phase-plate elements. Most of this structure is smoothed out by SSD. The distance $D$ between the first zeros of the envelope is equal to 250 times the beam's diffraction limit (for the phase plate considered), i.e.,

$$
D=250 \times\left(2 \lambda F^{\#}\right) .
$$

For an $f$-number $\left(F^{\#}\right)$ of 17.5 and $\lambda=0.35 \mathrm{~mm}$, we have $D=$ $3 \mathrm{~mm}$, which should correspond to the diameter of the target. Current direct-drive designs for the NIF use targets about $30 \%$ larger, which would require a larger number of phase-plate elements (i.e., $\sim 350 \times 350$ for the above example). More phase-plate elements (of smaller size) would produce a small shift in nonuniformity to shorter wavelengths relative to the target size, but the shift would not be large enough to significantly affect the results presented in the next section.

Current phase-plate strategies involve the use of continuously varying phases ${ }^{5}$ and kinoforms ${ }^{6}$ to avoid the energy loss around the target associated with the sharp variations in binary phase plates. These will also provide greater control over the shape of the intensity envelope in the target plane. It remains to be determined what effect these new phase plates will have on short-wavelength nonuniformities. They can significantly affect long-wavelength structures (spherical harmonic modes $\ell<20$ ) through the envelope shape. (It should be emphasized that any phase-plate envelope shape will be slightly modified by SSD and polarization dispersion.) As target designs and phase-plate strategies develop, their characteristic features can be incorporated into the uniformity calculations to determine their effects.

\section{Polarization Dispersion}

A birefringent wedge placed at the end of each beamline will cause the speckle pattern from one polarization to shift relative to the other. ${ }^{7}$ If the shift is more than about $1 / 2$ of a speckle width (which is roughly the coherence length), then the effect is similar to adding two different random-intensity patterns. ${ }^{8}$ The rms nonuniformity will be reduced instantaneously by a factor of $\sqrt{2}$. This effect was demonstrated at LLE using a liquid crystal (LC) wedge, ${ }^{7}$ but any other birefringent material, such as KDP, could be used. Figure 62.25 shows the experimental results of how the speckle modulation from a phase plate has been reduced using the LC wedge. An analysis of the intensity fluctuations around a smooth envelope
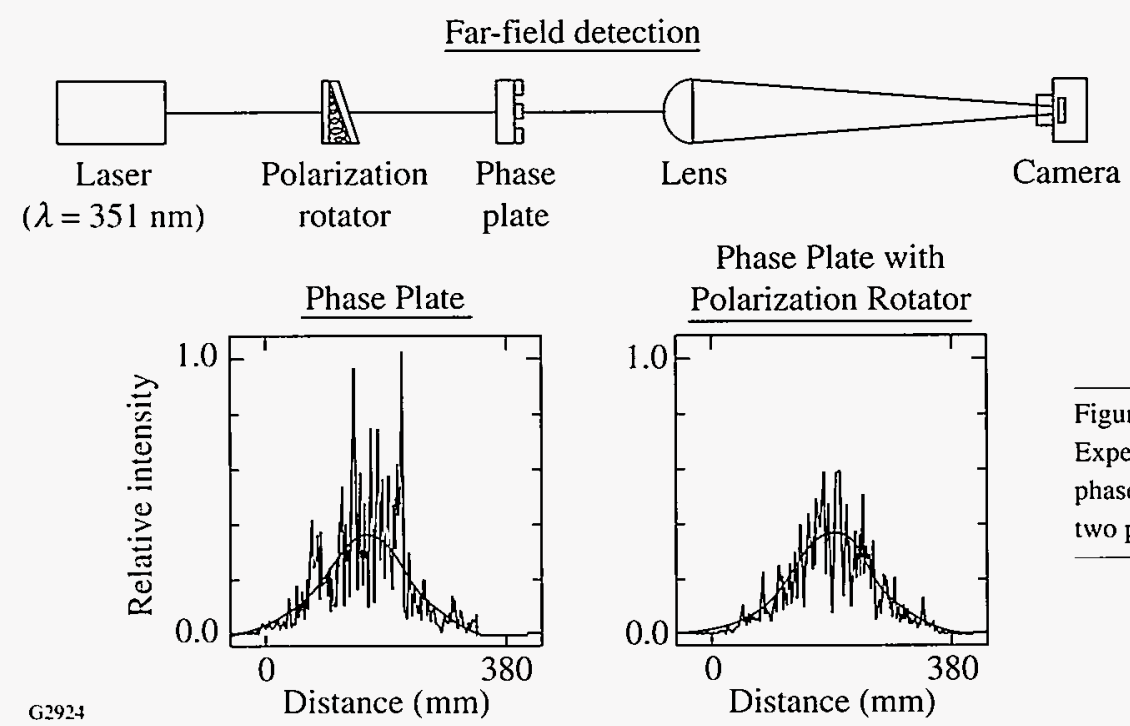

Phase Plate with

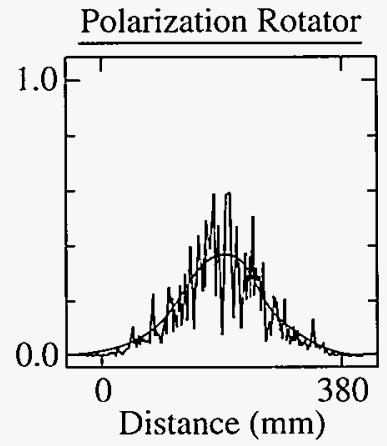

Figure 62.25

Experimentally observed reduction in nonuniformity from a phase plate using a birefringent wedge to spatially separate the two polarization components. 
showed that the rms variation was reduced by roughly a $\sqrt{2}$, as predicted. This superposition of shifted speckle patterns is similar to the smoothing mechanism produced by SSD, which shifts the speckle patterns for different spectral modes of the bandwidth. Optimized smoothing is achieved when the polarization and spectral shifts are complementary, as discussed below.

\section{SSD Considerations}

As the baseline configuration for 2-D SSD, we adopt the parameters described by Eimerl (Fig. 62.26). The one departure from that design, examined here, is the option for increased angular dispersion in one of the directions. It remains to be determined what effect such increased dispersion would have on laser performance and whether the amount of improved laser uniformity is large enough to justify a possible degradation in energy output.

One strategy for combining two-dimensional spectral dispersion of the bandwidth with polarization dispersion from a birefringent wedge is illustrated in Fig. 62.27, which shows the modal positions in the target plane. The minimum separation between modes should not be closer than $\sim 1 / 2$ the diffraction limit of the beam (i.e., $1 / 2$ a speckle size) for good smoothing. The symbols " $x$ " and " $o$ " represent orthogonal components of polarization. This configuration allows for the smallest amount of angular dispersion through the laser but requires the maximum polarization dispersion. To keep the beam nearly circular, either twice as many modes must be used in the direction perpendicular to polarization dispersion (i.e., twice the angular spread) or the beam envelope must be adjusted using the phase plate to compensate for unequal angular dispersion. ${ }^{1}$

To examine the effect of increasing the angular dispersion in one direction, we considered the example where the parameters associated with the 3-GHz modulator (Fig. 62.26) were kept constant and parameters of the other modulator were varied. The $1-\AA$ IR bandwidth from the $3-\mathrm{GHz}$ modulator produces about 31 spectral lines upon frequency tripling. These lines were spectrally dispersed to a separation of $1 / 2$ a speckle distance in the target plane, i.e., a total angular spread of 15 times the beam's diffraction limit (to be denoted by $15 x$ $D L)$. This was chosen to be the direction of polarization dispersion, which was also $15 \times \mathrm{DL}$, corresponding to the horizontal direction in Fig. 62.27. The total angular deflection in this direction is now $30 \times \mathrm{DL}$.

For spectral lines from the first modulator in Fig. 62.26, the modes were also dispersed to a separation of $1 / 2 \mathrm{DL}$ in the target plane, but the number of modes (i.e., the total angular spread) was allowed to vary while keeping the bandwidth constant. The bandwidth was maintained at a value near $4 \AA$ (IR) by varying the modulation frequency $v_{1}$ inversely with the number of spectral lines. (This bandwidth is about $10 \%$ lower than the value used by Eimerl.) A secondary constraint-that the frequencies from the two modulators should be incommensurate-could have been imposed. However, to simplify the calculation, it was only required that $v_{1}$ should be an odd multiple of $0.5 \mathrm{GHz}$. This approach assured that when its modes were combined with those from the $3-\mathrm{GHz}$ modulator, the resultant modes would have equally spaced frequencies separated by $\delta v=0.5 \mathrm{GHz}$. (The resultant modes have frequencies composed of a harmonic from one modulator plus a harmonic from the second.) Beam smoothing will then occur for averaging times up to $2 \mathrm{~ns}(1 / \delta v)$, which is the time required to smooth nearest-neighbor modes. If longer smoothing times are applicable, then $v_{1}$ can be chosen so that the resultant modes are more closely spaced in frequency.

Figure 62.28 shows the effect of increasing the angular dispersion in one direction. The rms nonuniformity for a single beam versus averaging time is plotted. Here, the rms nonuniformity is defined as the intensity fluctuation around the

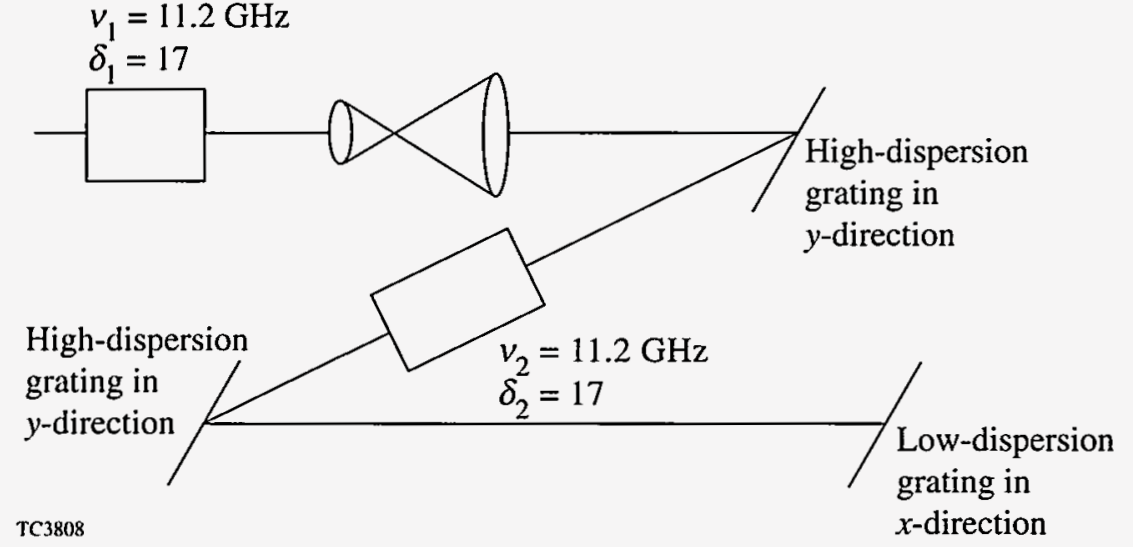

Figure 62.26

Configuration for 2-D SSD proposed for the NIF laser (Ref. 4). Here $v_{1}$ and $v_{2}$ are the modulation frequencies for the two modulators, and $\delta_{1}$ and $\delta_{2}$ are the modulation indices, corresponding to $1 / 2$ the number of spectral modes. 


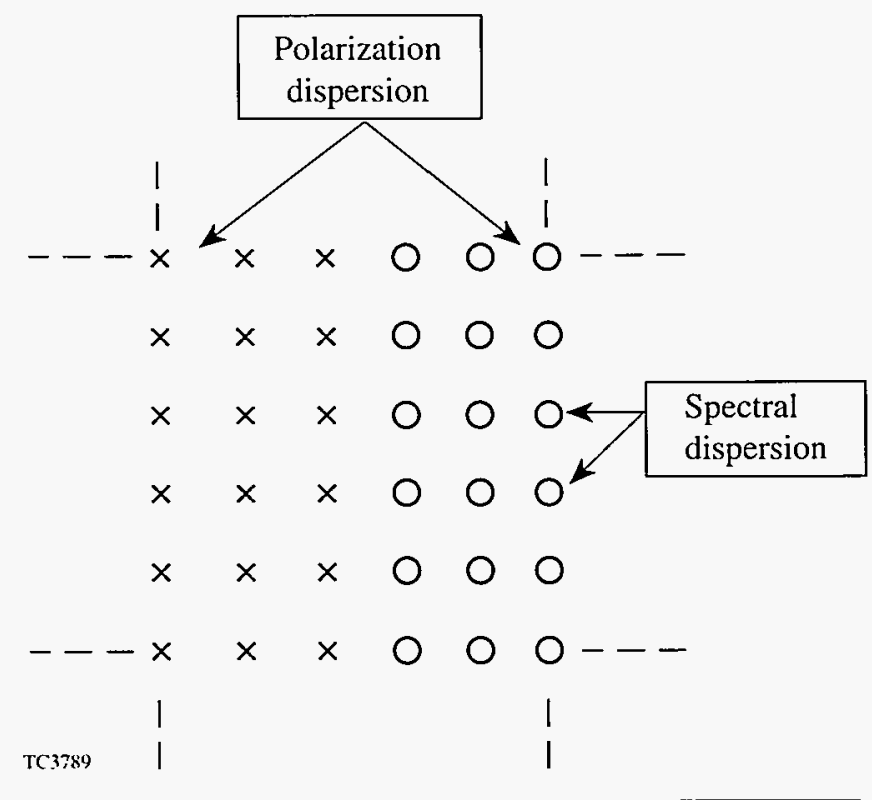

Figure 62.27

Strategy for combining 2-D SSD with polarization dispersion. The " $x$ " and "o" positions represent the locations of the dispersed spectral modes for the two polarization components. The components should be separated by at least $1 / 2$ of a speckle distance.

diffraction-limited envelope that would be produced if interference between all phase-plate elements were absent. The rms value was evaluated at the center of the beam, over a square region given by \pm 0.1 times the target radius in each direction. This gives a good sampling of the short-wavelength structure. (Long-wavelength nonuniformity is more appropriately discussed in conjunction with the effects of multiple-beam overlap.) The three curves in Fig. 62.28 are labeled by the angular deflection (in terms of the beam's UV diffraction limit) in each direction.

The single-beam results in Fig. 62.28 show that if we are interested only in averaging times less than $\sim 500 \mathrm{ps}$, then there is little advantage to increasing the angular dispersion. However. if averaging times of $\sim 1 \mathrm{~ns}$ or larger are relevant, then substantial improvements in uniformity can be achieved from increased dispersion. The majority of the effect is achieved at $30 \times \mathrm{DL}$ : For a $2-n s$ averaging time, the rms nonuniformity is reduced by $40 \%$, compared with the $15 \times$ DL result. (For $60 \times$ DL. the reduction is increased to $65 \%$.) The improved uniformity is probably the result of smoothing some of the longer-wavelength nonuniformities that become accessible with increased angular dispersion. (All three cases in Fig. 62.28 use the same bandwidth.) Table 62.III summarizes some of the SSD parameters and results.

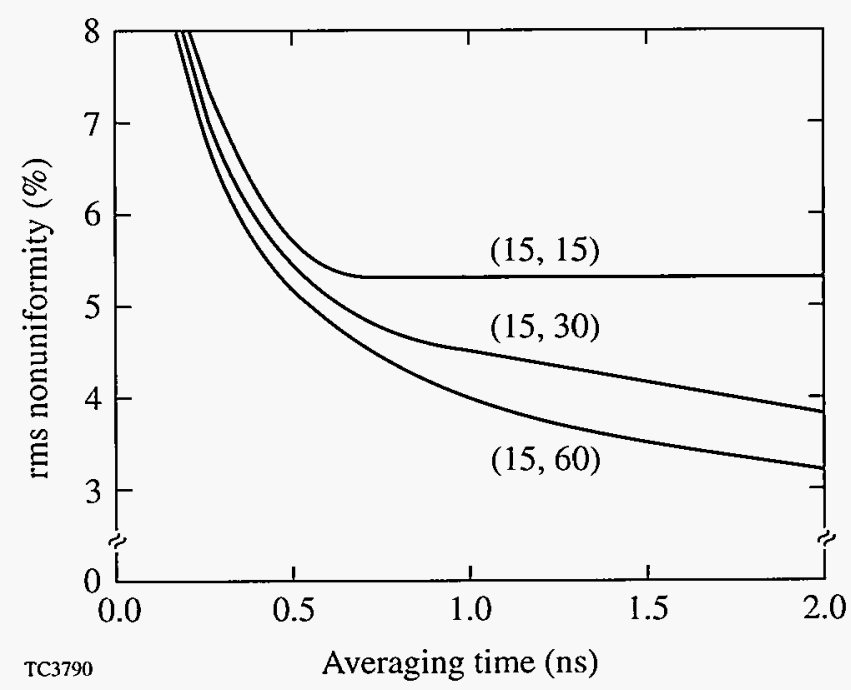

Figure 62.28

Improved irradiation nonuniformity with increased pinhole size. The numbers in parentheses indicate the angular dispersion for the two perpendicular directions, in terms of the beam's UV diffraction limit.

The uniformity results for spherical illumination, using multiple beam overlap, are shown in Fig. 62.29, which uses the configuration of 48 clusters of 4 beams from Table 62.11 with the pointing correction for the polar ring of beams. The axes of the beams in each cluster were also shifted by $20 \%$ of the target radius (using wedges) to produce an intensity envelope for the cluster that was less center-peaked than for the individual beams. (This could also be accomplished by using the phase plate to shape the envelope.) The beams were mapped directly onto the target surface, and the nonuniformity was expressed in terms of spherical harmonics for modes up to 512. No refractive smoothing or thermal smoothing in a plasma atmosphere was used, except indirectly by neglecting the veryshort-wavelength structure with spherical harmonic modes greater than 512. Interference between the beams was not included in the calculation. This is justified for the interference between beams within a cluster and between beams from different clusters with different frequencies, as this structure will smooth within $\sim 5 \mathrm{ps}$. Interference between beams (of the same frequency) from different clusters was not considered because this nonuniformity has spatial wavelengths that are much shorter than the minimum considered here. Such veryshort-wavelength nonuniformity would be smoothed by thermal conduction within the target, over distances of only a few microns. 
Table 62.III: Parameters related to the large-bandwidth modulator and the resultant single-beam rms nonuniformity.

\begin{tabular}{|c|c|c|c|}
\hline \multirow{2}{*}{} & \multicolumn{3}{|c|}{ Total Angular Disperson $\times$ DL) } \\
\cline { 2 - 4 } & 15 & 30 & 60 \\
\hline Modulation frequency $(\mathrm{GHz})$ & 13.50 & 6.5 & 3.5 \\
\hline Bandwidth $(\AA, \mathrm{IR})$ & 4.05 & 3.9 & 4.2 \\
\hline Single-beam nonuniformity & & & \\
$\Delta t=500$ ps & $5.9 \%$ & $5.7 \%$ & $5.4 \%$ \\
$\Delta t=2 \mathrm{~ns}$ & $5.3 \%$ & $3.9 \%$ & $3.3 \%$ \\
\hline \hline
\end{tabular}

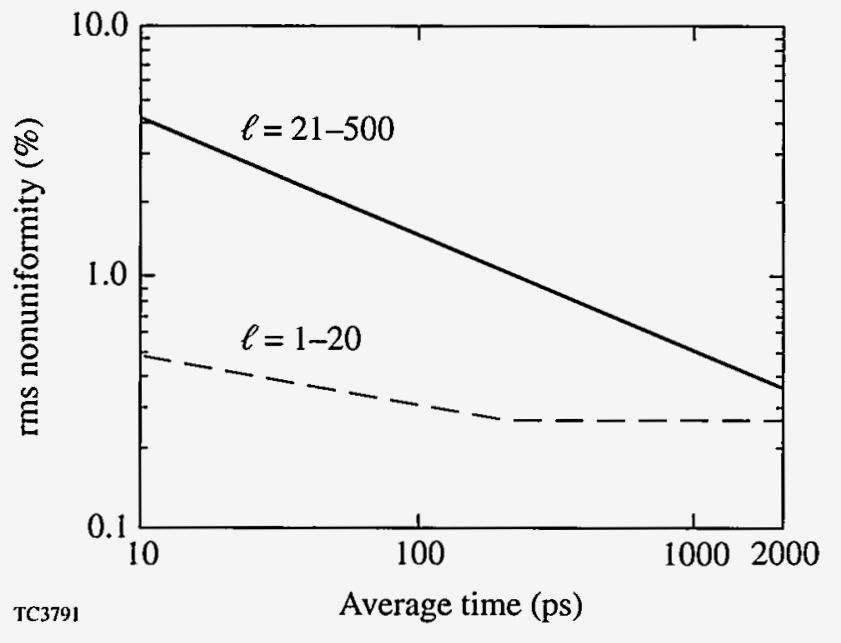

Figure 62.29

Irradiation nonuniformity for spherical irradiation as a function of averaging time. The $\ell$ values indicate the spherical harmonic modes for the two curves shown.

The nonuniformity for spherical irradiation (Fig. 62.29) has been separated into a long-wavelength component, with spherical harmonic modes $\ell \leq 20$, and a shorter-wavelength component with $21 \leq \ell \leq 512$. The long-wavelength modes are relatively static and are dominated by the shape of the cluster intensity envelope and the number of clusters. It is possible that this component of nonuniformity could be further reduced by modifying the envelope shape as part of the phase-plate design. The shorter-wavelength modes (21-512) decrease with averaging time $t$ roughly as $t^{-1 / 2}$. This is the same scaling as $\mathrm{ISI}^{9}$ and is obtained from the superposition of different random speckle patterns.

Finally, it should be pointed out that there is still additional nonuniformity present, with $\ell>512$, from structure in the individual beams. As an example, Fig. 62.30 shows the modal decomposition for an averaging time of $2 \mathrm{~ns}$. The nonuniformity clearly extends even above 512 . The largest mode number that should be present can be estimated as follows: The largest mode $\ell_{\max }$ is related to the wavelength of the smallest structure $\delta \lambda$ and to the target diameter by

$$
\ell_{\max }=\pi D / \delta \lambda
$$

The smallest wavelength should correspond roughly to the beam's diffraction limit (i.e., one speckle). Since $D$ is 250 times larger for this calculation, we have $\ell_{\max } \sim 800$. It remains to be determined what effect the very-short-wavelength structure has on target performance.

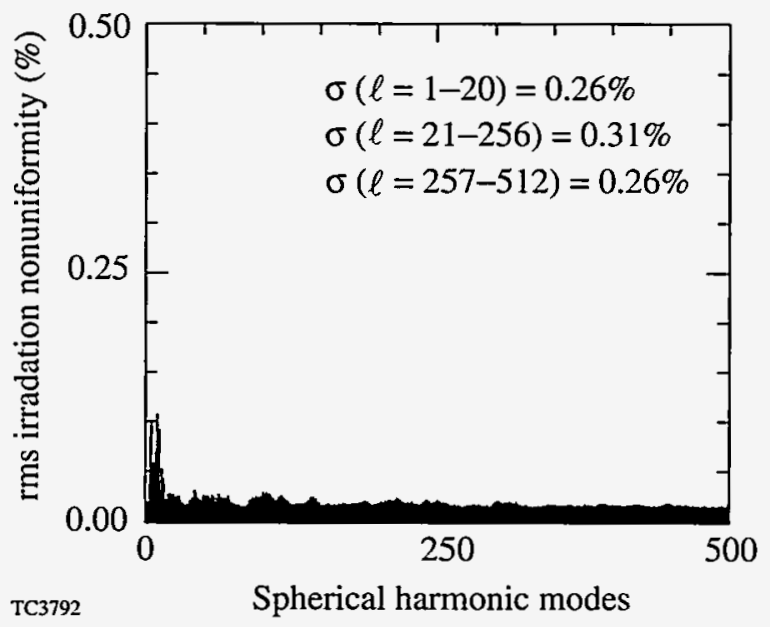

Figure 62.30

Spherical harmonic decomposition of the nonuniformity from Fig. 62.29 for 2-ns averaging time. 


\section{Summary}

The NIF can produce highly uniform irradiation for directdrive experiments if one-half of the beams are redirected to new ports closer to the "equator" of the target chamber and if 2-D SSD is implemented with polarization dispersion. The tolerances for energy imbalance among the beams, beam mispointing, and errors in target positioning depend on how much long-wavelength nonuniformity (spherical harmonic modes 1-4) the target can accept without serious degradation in performance. Using $1 \%$ for the maximum nonuniformity in these modes, we have the following constraints: (1) energy imbalance among the beams should be less than $\sim 5 \%$; (2) cluster mispointing should not exceed $\sim 5 \%$ of the target radius; and (3) the target positioning error should be less than $\sim 2 \%$ of the target radius.

The optimal pinhole size for SSD is another issue that depends on the target design. If intensity-averaging times greater than $\sim 1$ ns are of interest, then there is a definite advantage for the pinhole to be able to accommodate a divergence of $30 \times \mathrm{DL}$ in one direction and $15 \times \mathrm{DL}$ in the other for UV irradiation. It would be useful to determine what effect this would have on laser performance. If these divergences are available, it might be possible to optimize target designs to take advantage of the resultant higher uniformity for longer smoothing times.

\section{ACKNOWLEDGMENT}

This work was supported by the U.S. Department of Energy Office of Inertial Confinement Fusion under Cooperative Agreement No. DE-FC0392SF19460. the University of Rochester, and the New York State Energy Research and Development Authority. The support of DOE does not constitute an endorsement by DOE of the views expressed in this article.

\section{REFERENCES}

1. S. Skupsky, R. W. Short, T. Kessler, R. S. Craxton, S. Letzring, and J. M. Soures, J. Appl. Phys. 66, 3456 (1989).

2. J. W.-K. Mark, Phys. Lett. 114A, 458 (1986).

3. D. Eimerl (private communication).

4. D. Eimerl, presented at the 5th Meeting of the Inertial Confinement Fusion Advisory Committee/Defense Programs (ICFAC/DP), University of Rochester, Rochester, NY, 18-20 May 1994.

5. T. J. Kessler, Y. Lin, J. J. Armstrong, and B. Velazquez, in Laser Coherence Control: Technology and Applications, edited by H. T. Powell and T. J. Kessler (SPIE, Bellingham, WA, 1993), Vol. 1870, pp. 95-104.

6. S. N. Dixit et al., Opt. Lett. 19, 417 (1994).

7. Laboratory for Laser Energetics LLE Review 45, NTIS document No. DOE/DP40200-149, 1990 (unpublished), p. 1.

8. S. N. Dixit (private communication); also J. W. Goodman in Laser Speckle and Related Phenomena, Topics in Applied Physics, Vol. 9, edited by J. C. Dainty (Springer-Verlag, Berlin, 1984), Chap. 2, pp. 9-75.

9. R. H. Lehmberg, A. J. Schmitt, and S. E. Bodner, J. Appl. Phys. 62, 2680 (1987). 


\section{Direct Measurements of Terminal-Level Lifetime in Nd:YLF}

The terminal-level lifetime of a four-level laser fundamentally affects its gain saturation performance since slow relaxation rates from this level can form a "bottleneck" when the saturating pulses are significantly shorter than the terminal-level lifetime. As a result, the terminal-level lifetime is an important design parameter in achieving maximum energy extraction from high-peak-power laser amplifiers and $Q$-switched oscillator systems.

Terminal-level lifetimes reported in the literature for even the most common laser media such as Nd:YAG differ by as much as several orders of magnitude; ${ }^{1-4}$ references for $\mathrm{Nd} \mathrm{YLF}^{2,3}$ are less common and subject to the same uncertainty.

For most solid-state laser materials, the terminal-level lifetime is dominated by nonradiative relaxation processes involving the emission or absorption of host material phonons. Closely spaced energy levels within a Stark manifold thermalize extremely rapidly $(<10 \mathrm{ps})^{5,6}$ through nonradiative processes involving single phonons, while relaxation between different Stark manifolds proceeds at significantly slower rates since multiple phonons are required to bridge the typically larger intermanifold energy gaps. When the total multiphonon, nonradiative relaxation out of the terminal laser level manifold is much slower than the intramanifold thermalization, the distribution of population within the manifold can be treated as a system in quasi-thermal equilibrium and described by a Boltzmann distribution. For laser systems in which the terminal laser level contains a significant proportion of its thermalized manifold population, gain saturation properties are determined by both the relaxation rate of the entire manifold and the quasi-thermal occupation factor in the terminal laser level. The manifold relaxation rate, often characterized by its lifetime, determines whether any "bottlenecking" occurs, and the thermal occupation factor determines the degree to which accumulation of population in the terminal-level manifold reduces population inversion during lasing.

Measured lifetimes for other energy levels can yield relevant information since multiphonon nonradiative relaxation rates generally follow a phenomenological model 7,8 that depends only on temperature, energy gap to the next lower energy level, and a single phonon frequency. Measured relaxation rates for high-order phonon processes $(n>3)$ substantially support this model $; 9,10$ however, it is generally invalid for loworder processes $(n \leq 3)$. Anomalies have also been observed in some materials ${ }^{8}$ due to selection rules or when a high degree of resonance exists between an intermanifold energy gap and a sum of phonon energies corresponding to peaks in the host material's phonon spectrum. In addition, significantly different lifetimes for nonradiative transitions covering nearly identical energy gaps have been reported for $\mathrm{Nd}: \mathrm{YAG}^{3,11}$ that suggest either a breakdown in the model or a dependence on the measurement technique.

Direct measurement of the ${ }^{4} I_{11 / 2}$ terminal-level relaxation is performed ideally under prototypical energy extraction conditions to ensure meaningful results. Sensitive and accurate measurement of the relaxation rate demands operating in a regime that clearly isolates the multiphonon nonradiative decay process from radiative processes. Saturating pulses substantially shorter than the terminal-level lifetime can yield a true impulse response that is easily analyzed without the need for deconvolving input beam pulse shapes or applying complicated models of the population dynamics. Any practical method should by design also minimize the amount of sample required and avoid nonlinear optical processes, such as self-focusing or self-phase modulation, that might damage the sample or otherwise introduce unquantifiable effects into the measurement. ${ }^{12}$

\section{Theory}

Figure 62.31 represents the energy levels in Nd:YLF important to laser action. The $1475-\mathrm{cm}^{-1}$ energy gap ${ }^{13}$ between the ${ }^{4} I_{11 / 2}$ terminal-level manifold and the ${ }^{4} I_{9 / 2}$ ground-state manifold is less than three times the phonon cutoff energy of $566 \mathrm{~cm}^{-1}$ in YLF, ${ }^{14}$ which places this nonradiative decay process near the limit of validity for the single-frequency model.

In this work, the ${ }^{4} F_{3 / 2}$ initial-laser-level manifold is pumped directly from the ${ }^{4} I_{9 / 2}$ ground-state manifold. Laser action at 


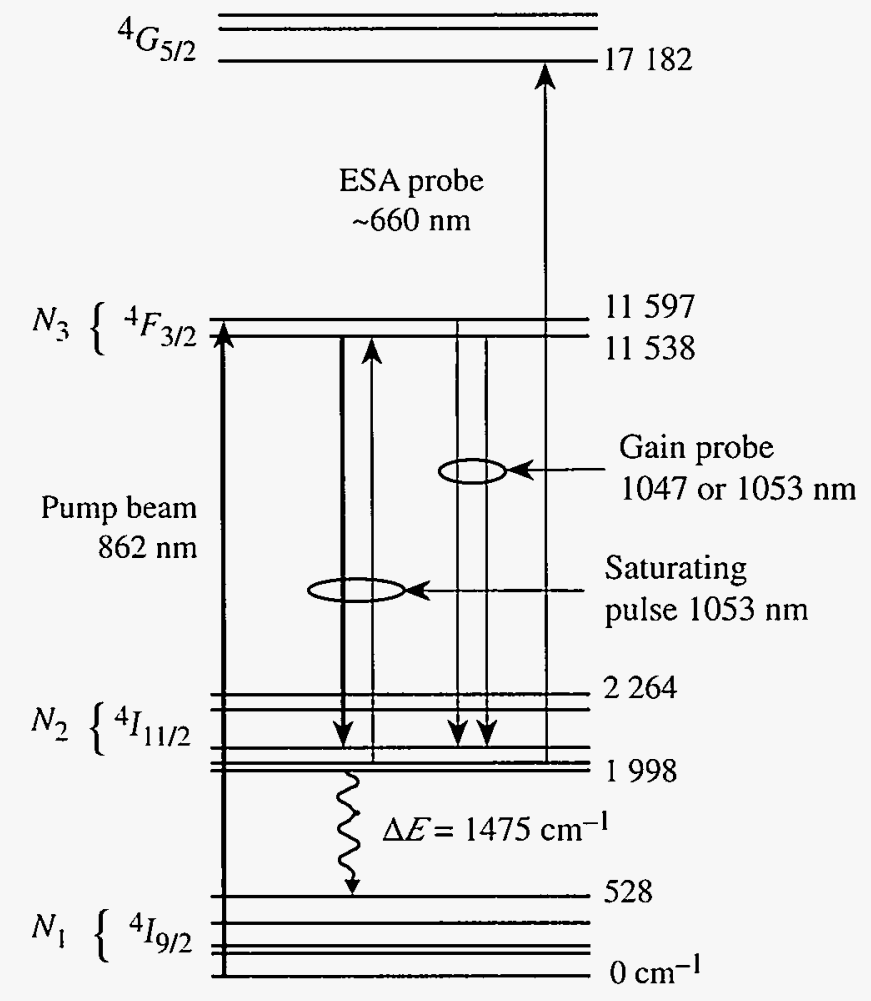

E7+46

Figure 62.31

Energy-level diagram for lasing in Nd:YLF. The ${ }^{4} / 9 / 2$ ground state and $+/ 11 / 2$ terminal laser state are separated by a $1475-\mathrm{cm}^{-1}$ energy gap that is less than three times the maximum phonon energy in $\mathrm{YLF}^{14}\left(\sim 566 \mathrm{~cm}^{-1}\right)$. The thick upward (downward) arrow represents the pump (saturating pulse) laser transitions. The thin downward (upward) arrow represents the small-signalgain (excited-state absorption) probe transitions.

the 1053-nm laser transition occurs between the lowest sublevel in the ${ }^{4} F_{3 / 2}$ manifold and the second lowest level in the ${ }^{4} I_{11 / 2}$ manifold. Since both the initial and terminal laser levels are strongly coupled to their respective Stark manifolds and each contains a substantial portion of its manifold's population, the relevant population dynamics are best described by rate equations for the total populations $N_{3}$ and $N_{2}$ of the initial $\left({ }^{4} F_{3 / 2}\right)$ and terminal $\left({ }^{4} I_{11 / 2}\right)$ laser-level manifolds, respectively.

$$
\begin{aligned}
\frac{d N_{3}(\bar{r}, t)}{d t} & =W_{\text {pump }}(t) \cdot N_{1}(\bar{r}, t)-W_{\text {sat pulse }}(t) \\
& \times\left[f_{3} N_{3}(\bar{r}, t)-f_{2} N_{2}(\bar{r}, t)\right]-N_{3}(\bar{r}, t) / \tau_{3},
\end{aligned}
$$

$$
\begin{aligned}
\frac{d N_{2}(\bar{r}, t)}{d t} & =W_{\text {sat pulse }}(t) \\
& \times\left[f_{3} N_{3}(\bar{r}, t)-f_{2} N_{2}(\bar{r}, t)\right]-N_{2}(\bar{r}, t) / \tau_{2},
\end{aligned}
$$

where $W_{\text {pump }}$ and $W_{\text {sat pulse }}$ are the radiative transition rates associated with the pump and saturating pulses, respectively, given by $W(t)=I(t) \cdot \sigma / h v$, where $I(t)$ is the intensity, $\sigma$ is the corresponding pump absorption or laser-stimulated emission cross section, and $h v$ is the photon energy; $\tau_{3}$ and $\tau_{2}$ are the lifetimes of the initial and terminal-laser-level manifolds, respectively; and the thermal occupation factors of the actual Stark sublevels of the laser transition, $f_{3}$ and $f_{2}$, are given by $f_{i}=e^{-E_{1} / k T} / Z_{i}$, where $E_{i}$ is the energy of the sublevel within the manifold and $Z_{i}$ is the partition function for the manifold. Given that no other energy levels accumulate significant populations, all relevant population dynamics are described by Eq. (1) plus the closure condition $N_{0}=N_{1}+N_{2}+N_{3}$, where $N_{0}$ is the doping concentration of the crystal and $N_{1}$ is the total population of the ${ }^{4} I_{9 / 2}$ ground-state manifold.

The rapid thermalization of the initial and terminal laser levels within their respective Stark manifolds can also be addressed in analytic solutions for laser-energy extraction performance ${ }^{15}$ when saturating pulse lengths are short compared to the lifetime of the entire terminal-level manifold but long compared to the intramanifold thermalization processes. In a manner analogous to applying level degeneracy factors, ${ }^{16}$ the expression for saturation fluence can be modified to include the thermal occupation factors $F_{\text {sat }}=h v /\left(f_{3}+f_{2}\right) \sigma_{\text {se }}$, where $\sigma_{\mathrm{se}}$ is the spectroscopic stimulated emission crosssection. This expression reduces to the well-known value of $F_{\text {sat }}=h v / \sigma_{\text {se }}$ for an ideal four-level system $\left(f_{3}=1, f_{2}=0\right)$, and $F_{\text {sat }}=h v / 2 \sigma_{\text {se }}$ for a three-level system $\left(f_{3}=1, f_{2}=1\right)$. Simple analytical models are inadequate for the intermediate case where laser pulse lengths are comparable to the terminallaser-level manifold lifetime.

Figure 62.31 also shows small-signal-gain and excitedstate absorption probes used in measuring the terminal-level lifetime. The small-signal-gain probe measures the population inversion $\Delta N=f_{3} N_{3}-f_{2} N_{2}$, while the excited-state absorption probe monitors directly the population of the terminal manifold. ${ }^{12,17}$ Assuming a plane-wave interaction, the time dependence of small-signal probe beams propagating in the $x$ direction are determined by the population dynamics of the initial and terminal laser levels according to simple exponential growth and absorption laws 


$$
\begin{gathered}
G_{\mathrm{ss}}(t)=\exp \left\{\int_{x=0}^{L} \Delta N(x, t) \sigma_{\mathrm{se}} d x\right\}, \\
T_{\mathrm{ESA}}(t)=\exp \left\{-\int_{x=0}^{L} f_{2} N_{2}(x, t) \sigma_{\mathrm{ESA}} d x\right\},
\end{gathered}
$$

where $\sigma_{\mathrm{se}}$ and $\sigma_{\mathrm{ESA}}$ are the stimulated emission and excitedstate absorption cross sections, respectively, and $L$ is the sample thickness. The exponential arguments are integrated along the path traveled in the sample by the probe beams since the populations may vary spatially. Zero population in the ${ }^{4} G_{5 / 2}$ upper level of the excited-state absorption transition is assumed in Eq. (2b) for small probe signals.

After the initial-laser-level pumping has stopped and after an impulse-like saturating pulse has extracted stored energy at time $t_{0}$, Eqs. (1a) and (1b) become uncoupled differential equations with simple exponential solutions

$$
N_{3}(x, t)=N_{3}\left(x, t_{0}\right) \cdot \exp \left[-\left(t-t_{0}\right) / \tau_{3}\right]
$$

and

$$
N_{2}(x, t)=N_{2}\left(x, t_{0}\right) \cdot \exp \left[-\left(t-t_{0}\right) / \tau_{2}\right]
$$

where $N_{3}\left(x, t_{0}\right)$ and $N_{2}\left(x, t_{0}\right)$ are the population densities of the initial and terminal laser manifolds, respectively, left by the saturating pulse at $t_{0}$.

Substituting into Eq. (2) gives

$$
\begin{gathered}
G_{\mathrm{sS}}\left(t>t_{0}\right)=G_{\text {final }} \cdot \exp \left\{-\alpha_{\mathrm{se}}\left(t_{0}\right) L \cdot e^{-\left(t-t_{0}\right) / \tau_{2}}\right\}, \\
T_{\mathrm{ESA}}\left(t>t_{0}\right)=\exp \left\{-\alpha_{\mathrm{ESA}}\left(t_{0}\right) L \cdot e^{-\left(t-t_{0}\right) / \tau_{2}}\right\},
\end{gathered}
$$

where

$$
\alpha_{\mathrm{se}}\left(t_{0}\right)=1 / L \int_{x=0}^{L} f_{2} N_{2}\left(x, t_{0}\right) \sigma_{\mathrm{se}} d x
$$

and

$$
\alpha_{\mathrm{ESA}}\left(t_{0}\right)=1 / L \int_{x=0}^{L} f_{2} N_{2}\left(x, t_{0}\right) \sigma_{\mathrm{ESA}} d x
$$

represent the effective excited-state absorption coefficients for the small-signal probes at time $t_{0}$. Since the ${ }^{4} F_{3 / 2}$ initial laserlevel lifetime is extremely long compared to the lower-level lifetime, ${ }^{6,18}$ the upper-laser-level population can be taken as constant with a value $N_{3}(t) \sim N_{3}\left(t_{0}\right)$. The final small-signal gain after relaxation of the terminal laser level is given by

$$
G_{\text {final }}=\exp \left\{\int_{x=0}^{L} f_{3} N_{3}\left(x, t_{0}\right) \sigma_{\text {se }} d x\right\} .
$$

\section{Experimental Setup}

Figure 62.32 illustrates the experimental setup. Thin Nd:YLF samples approximately 1 and $2 \mathrm{~mm}$ thick with nominal 1 -at.-\% doping concentrations are intracavity pumped directly into the ${ }^{4} F_{3 / 2}$ initial-laser-level manifold by a free-running, tunable Cr:LiSAF pulsed laser. The pump laser is tuned to the weak Nd:YLF absorption band at $\sim 860 \mathrm{~nm}$ using a birefringent tuning filter made from four crystalline quartz plates positioned at Brewster's angle. Horizontal polarization is further enforced by orienting the stronger $\pi$-polarization emission of the Cr:LiSAF rod to match the low-loss horizontal polarization of the Brewster tuning plates. The crystalline axis of the $\mathrm{Nd}$ :YLF sample is also horizontal to match the horizontal pump polarization with the stronger $\pi$-polarization absorption for Nd:YLF ${ }^{18}\left(\alpha_{\max } \approx 0.3 \mathrm{~cm}^{-1}\right)$. Operation in the $\mathrm{TEM}_{00}$ mode is accomplished with an intracavity aperture in the nearly hemispherical resonator. Pump pulses with overall durations of approximately $70 \mu \mathrm{s}$ and numerous relaxation oscillations were obtained typically, as shown in Fig. 62.33. Uniform pumping is expected since the sample is optically thin at this wavelength. Furthermore, multiple-longitudinal-mode operation of the pump laser should smooth out any spatial hole burning that might occur early during the pump pulse. The diameter of the Nd:YLF sample's pumped region is approximately $1 \mathrm{~mm}$ in the vertical direction and elongated in the horizontal direction since it is also positioned at Brewster's angle inside the pump laser cavity to minimize reflection losses from the uncoated sample.

A saturating pulse is generated from an $\sim 70$-ps (FWHM) pulse picked from the output of a cw mode-locked Nd:YLF laser operating at $1053 \mathrm{~nm}$ and amplified to the millijoule regime in an unstable, regenerative ring amplifier. ${ }^{19}$ The 


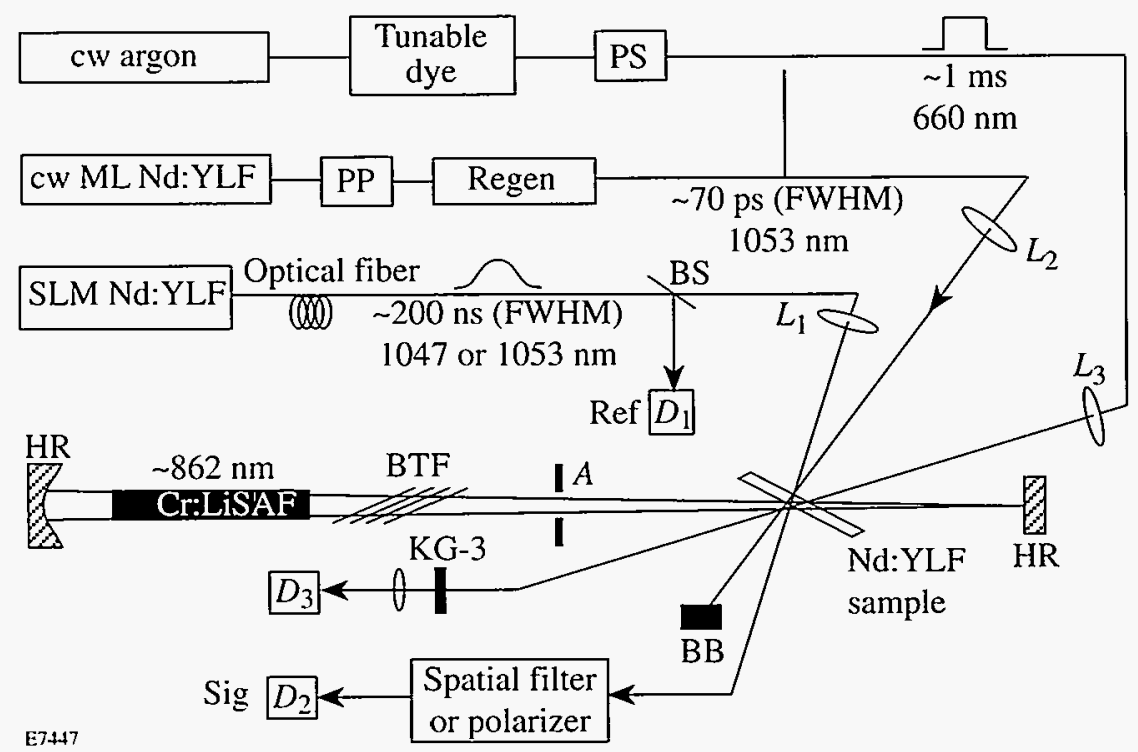

Figure 62.32
Experimental setup for measurement of terminal-
level lifetimes; $L_{1}, L_{2}$, and $L_{3}=$ focusing lenses; $D_{1}$,
$D_{2}$, and $D_{3}=$ fast silicon photodiodes; BTF =
birefringent tuning filter; $\mathrm{HR}=$ high reflector mir-
rors; $\mathrm{BB}=$ beam block; $\mathrm{BS}=$ beam splitter; $\mathrm{PP}=$
pulse picker; PS = pulse slicer; SLM = single-
longitudinal-mode laser; $A=$ mode-controlling
aperture; KG-3 = glass filter.

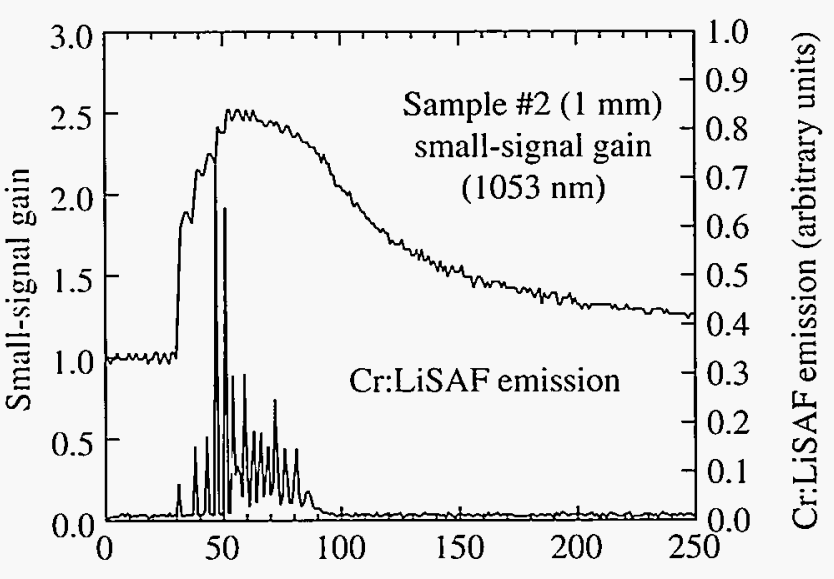

E74+48

Time ( $\mu s)$

Figure 62.33

The time evolution of the Cr:LiSAF pump laser and the single-pass, smallsignal gain through a pumped. 1-mm sample. Strong saturation of the pump absorption is observed after the first relaxation oscillation. The small-signal gain decays after termination of the pump pulse according to a double exponential characterized by an early time constant $\tau=30 \mu$ s and a long time constant $\tau=200 \mu \mathrm{s}$.

saturating pulse is cavity dumped from the regenerative amplifier and focused to $\sim 400-\mu \mathrm{m}$ diameter in the center of the sample's pumped region to achieve high saturation fluences. The polarization of the saturating pulse at the sample is vertical to align it with the $1053-\mathrm{nm}$ ( $\sigma$-polarization) axis of the Nd:YLF sample.

The small-signal-gain recovery of the Nd:YLF sample is measured using the fiber-coupled output of a $Q$-switched, monomode Nd:YLF oscillator operating at either 1047 or $1053 \mathrm{~nm}$. The approximately 200-ns FWHM, bell-shaped pulses are split into signal and reference beams that were measured by fast silicon photodiodes ( 150 -ps rise time) using an HP 54720A fast digitizing oscilloscope (4 Gsamples/s, 1-GHz bandwidth). The signal-to-reference beam ratios were calculated for each shot to monitor the evolution of the singlepass, small-signal gain and then averaged over a number of shots. The small-signal-gain probe beam is focused to approximately a $20-\mu \mathrm{m}$ spot to spatially sample only the center of the saturated gain region. When using the $1053-\mathrm{nm}$ probe beam, this spot was image relayed through a spatial filter to minimize light scattered from the saturating pulse into the solid angle of the signal-detector collection optics. For measurements with the $1047-\mathrm{nm}$ probe beam, the probe beam polarization was orthogonal to the saturating pulse, and a thin-film polarizer was used to reject the scattered light. The actual single-pass, small-signal gain was obtained by removing passive effects, such as Fresnel reflections, and scattering in the sample measured by the signal beam when the sample was unpumped.

The transient excited-state absorption from the ${ }^{4} I_{11 / 2}$ terminal level was measured with a cw dye laser using DCM Special Dye (Lambda Physik LC 6501). The dye laser was pumped by a $5-\mathrm{W} \mathrm{cw}$ argon ion laser, and the output was tuned to $660.4 \mathrm{~nm}$ using a three-plate, quartz birefringent tuning filter. A mechanical shutter was used to gate 1-ms square pulses. both to reduce the average power incident on the fast silicon photodiode and to preserve its fast response time ( 150 -ps rise time). A 2-mm-thick KG-3 Schott Glass filter was used to eliminate 1053-nm light scattered from the saturating pulse while passing the $660-\mathrm{nm}$ probe beam. The 
time-resolved measurements of optical transmission were averaged over a number of shots.

\section{Results and Discussion}

Figure 62.33 illustrates a typical evolution of the singlepass, small-signal gain at $1053 \mathrm{~nm}$ achieved in a 1-mm-thick sample by the pump laser. Peak single-pass, small-signal gains greater than 2.5 were achieved in 1-mm-thick samples, and gains in excess of 4.0 in a 2 -mm sample. The small-signal gain exhibits a double-exponential decay with lifetimes $(\tau \approx 30 \mu \mathrm{s}$ and $\tau \approx 200 \mu \mathrm{s}$ ) substantially shorter than published values for the fluorescence lifetime ${ }^{6,18}$ for these low-doped crystals; however, even the fast component of this decay is still extremely long compared to the terminal-level lifetime. The reduced fluorescence lifetime appears to be related to the extremely high population inversion densities and visible fluorescence observed from the samples, which is likely the result of up-conversion processes promoted by the intense pumping. Background small-signal gain and excited-state absorption measurements taken without the saturating pulse present revealed no effect on laser-level population dynamics related to the intense pumping or the observed up-conversion processes on the time scale of the terminal-level lifetime.

Figure 62.34 illustrates a typical small-signal-gain $\left(G_{\mathrm{ss}}\right)$ recovery measurement using the $1047-\mathrm{nm}$ probe beam. The small-signal gain measured for a single pass of the probe beam through the pumped sample drops rapidly as the saturat-

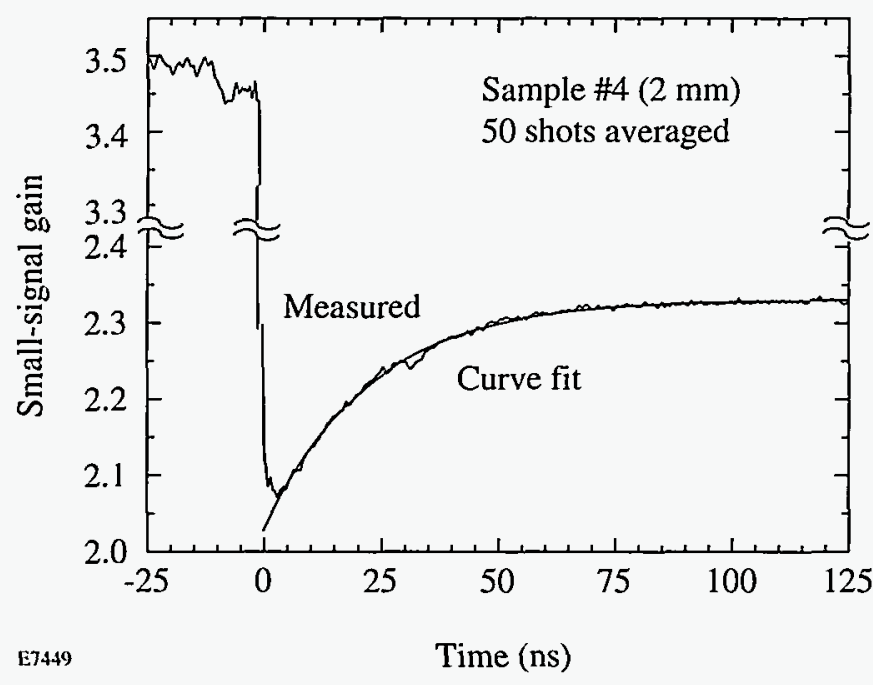

Figure 62.34

A typical small-signal-gain recovery measurement using the $1047-\mathrm{nm}$ probe beam. The small-signal gain of a saturated sample (\#4) recovers after passage of a short saturating pulse at $t=0$. (Note the split vertical scale.) Curve fits using Eq. (3a) for $t>0$ for different samples are given in Table 62.IV. ing pulse extracts stored energy at time $t=0$. The small dip observed at $t=-10 \mathrm{~ns}$ is caused by a prepulse leaked one roundtrip before the regenerative amplifier is cavity dumped. The smooth recovery after $t=0$ to an asymptotic value caused by relaxation of the terminal laser level can clearly be seen along with a nonlinear exponential best fit to Eq. (3a). Table 62.IV presents average curve-fit values for the terminallevel lifetime of three different samples using both the 1047- and 1053-nm probe beams, as well as the standard deviation of the curve-fit values. No variations were observed for different positions in the samples measured, and excellent agreement is found from sample to sample.

Figure 62.35 displays a typical transient excited-stateabsorption (ESA) measurement using another Nd:YLF sample. Larger transient ESA measurements were observed for $\pi$ polarized probe beams. The transmission through the pumped region of the sample is unity until the saturating pulse rapidly populates the terminal laser level at time $t=0$. The observed transmission of the sample decreases rapidly and then returns to its equilibrium value on a time scale governed by the terminal-level lifetime. A nonlinear best fit to $\mathrm{Eq}$. (3b) is also plotted. Again, evidence of a prepulse is observed at $t=-10 \mathrm{~ns}$. Table 62.IV also includes curve-fit values for the terminallevel lifetime obtained from transient ESA measurements on several samples that are in excellent agreement with the values obtained from the small-signal-gain recovery measurements.

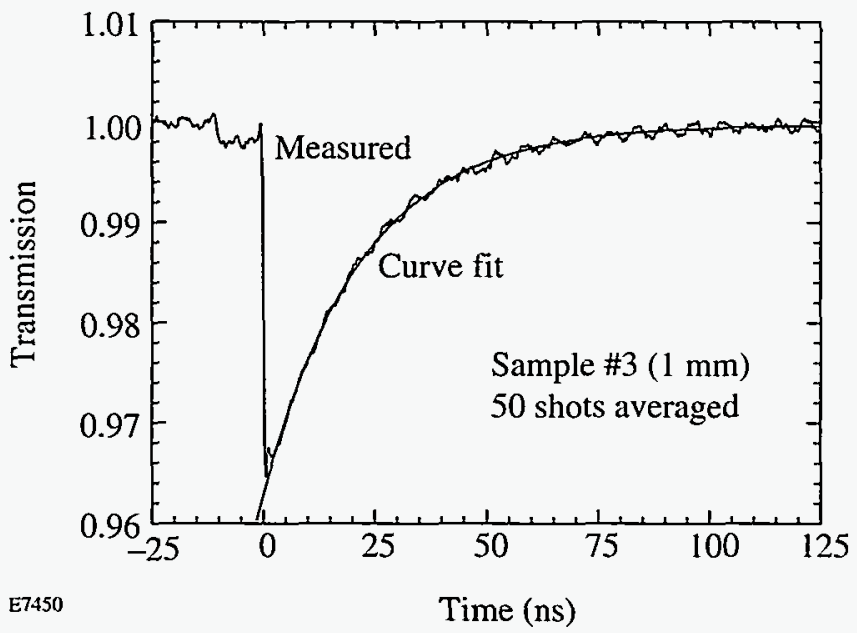

Figure 62.35

A typical ESA measurement in a different Nd:YLF sample (\#3). Transient excited-state absorption from the terminal level occurs after passage of a short saturating pulse at $t=0$. Curve fits using Eq. (3b) for $t>0$ for different samples are given in Table 62.IV. 
Table 62.IV: Measured values for the terminal-level lifetime of four different Nd:YLF samples using small-signal-gain $\left(G_{\mathrm{SS}}\right)$ recovery and transient excited-state absorption (ESA) methods are consistent within experimental accuracy.

\begin{tabular}{|c|c|c|c|c|}
\hline \hline Sample & Thickness (mm) & Lifetime (ns) & $\begin{array}{c}\text { Standard } \\
\text { Deviation (ns) }\end{array}$ & Method \\
\hline 1 & 1.015 & 21.9 & \pm 0.2 & $1047-\mathrm{nm} G_{\mathrm{ss}}$ recovery \\
\hline 2 & 1.100 & 21.9 & \pm 0.2 & $1047-\mathrm{nm} G_{\mathrm{sS}}$ recovery \\
& 21.4 & 21.8 & \pm 0.4 & 1053 -nm $G_{\mathrm{sS}}$ recovery \\
& & 21.4 & \pm 0.2 & transient ESA \\
\hline 3 & 1.435 & 21.3 & \pm 0.2 & transient ESA \\
\hline 4 & 2.230 & 21.7 & \pm 0.3 & 1047 -nm $G_{\mathrm{SS}}$ recovery \\
& & & \pm 0.7 & transient ESA \\
\hline \hline
\end{tabular}

\section{Conclusions}

The terminal-level lifetime for Nd:YLF measured in this work is long compared to the pulse lengths encountered for mode-locked laser operation and amplification of up to nanosecond pulses. In these cases, Nd:YLF must be treated as a three-level system amenable to standard Frantz-Nodvik gain saturation analysis with a modified expression for the saturation fluence that accounts for rapid thermalization of the initial and terminal laser levels within their respective Stark manifolds. Since common $Q$-switched laser pulse lengths are comparable to the terminal-laser-level lifetime measured in this work, simple analytical models are inadequate to account for terminal-level relaxation during amplification of such pulses, and numerical solutions are required to calculate energy-extraction performance.

\section{ACKNOWLEDGMENTS}

The authors wish to acknowledge Lightning Optical Corporation for providing the Nd:YLF samples. The authors also wish to thank S. A. Letzring for technical support related to high-speed instrumentation and electro-optic elements used throughout the experiments. This work was supported by the U.S. Department of Energy Office of Inertial Confinement Fusion under Cooperative Agreement No. DE-FC03-92SF19460 and the University of Rochester. The support of DOE does not constitute an endorsement by DOE of the views expressed in this article.

\section{REFERENCES}

1. F. E. Hovis et al. IEEE J. Quantum Electron. 28, 39 (1992).

2. K. Palombo et al., in OSA Proceedings on Advanced Solid-State Lasers, 1993. edited by A. A. Pinto and T. Y. Fan (Optical Society of America. Washington, DC, 1993). Vol. 15, pp. 78-80.

3. C. Bibeau. S. A. Payne, and H. T. Powell, in OSA Proceedings on Advanced Solid-State Lasers, 1994, edited by T. Y. Fan and B. H. T.
Chai (Optical Society of America, Washington, DC, 1994), Vol. 20 , pp. 74-76.

4. W. Koechner, Solid-State Laser Engineering, 2nd rev. and updated ed., Springer Series in Optical Sciences, Vol. 1 (Springer-Verlag, New York, 1988), p. 49.

5. T. Kushida, Phys. Rev. 185, 500 (1969).

6. A. L. Harmer, A. Linz, and D. R. Gabbe, J. Phys. Chem. Solids 30, 1483 (1969).

7. H. W. Moos, J. Lumin. 1,2, 106 (1970).

8. L. A. Riseberg and M. J. Weber, in Progress in Optics, edited by E. Wolf (North-Holland, New York, 1976), Vol. XIV, pp. 91-159.

9. M. J. Weber, Phys. Rev. B 8, 54 (1973).

10. H. P. Jenssen, Ph.D. thesis, Massachusetts Institute of Technology, 1971.

11. T. T. Basiev et al., J. Lumin. 53, 19 (1992).

12. N. E. Bykovskii et al., Sov. J. Quantum Electron. 18, 783 (1988).

13. A. A. S. da Gama et al., J. Chem. Phys. 75, 2583 (1981).

14. E. Schultheiss, A. Scharmann, and D. Schwabe, Phys. Stat. Sol. (b) 138, 465 (1986).

15. L. M. Frantz and J. S. Nodvik, J. Appl. Phys. 34, 2346 (1963).

16. A. E. Siegman, Lasers (University Science Books, Mill Valley, CA, 1986), pp. 153-157.

17. V. A. Sychugov and G. P. Shipulo, Sov. Phys. JETP 31, 438 (1970).

18. J. R. Ryan and R. Beach, J. Opt. Soc. Am. B 9, 1883 (1992).

19. D. Y. Park, W. Seka, Y. Lin, and D. L. Brown, in the Proceedings of the International Conference on Lasers ' 89 , edited by D. G. Harris and T. M. Shay (STS Press, McLean, VA, 1990), pp. 449-456. 


\section{Light-Emitting Porous Silicon: Materials Science, Properties, and Device Applications}

Silicon-based, light-emitting devices (LED's) should find numerous uses in optoelectronics. For example, the integration of silicon LED's with silicon microelectronics could lead to reliable and inexpensive optical displays and optical interconnects. Until recently, however, it had not been possible to obtain efficient room-temperature luminescence from silicon. The demonstration in 1990 that a form of silicon called "porous" can emit bright photoluminescence in the red region of the spectrum triggered worldwide research efforts aimed at establishing the mechanisms for the unexpected luminescence and at fabricating efficient and durable LED's. In less than five years, significant progress has been achieved on both fronts. LED's emitting throughout the visible spectrum have been demonstrated, and the best measured external electroluminescence efficiency has risen from $10^{-5} \%$ to better than $0.01 \%$ at room temperature. The photoluminescence efficiency of the best samples is near $10 \%$ at room temperature, and lightemitting porous silicon (LEPSi) that luminesces from the blue part of the spectrum to the infrared beyond $1.5 \mu \mathrm{m}$ has been produced. In this article, we first review why silicon is a poor light emitter and then define porous silicon and its main properties. We then focus on the properties of the three luminescence bands ("red," "blue," and "infrared") and present the results of femtosecond time-resolved optical measurements. Next, we report progress toward the fabrication of LED's and discuss some specific device structures. Finally, we outline what is necessary for commercial LEPSi LED's to become a reality and report on experimental results that suggest the possible integration of LEPSi with standard microelectronic devices.

\section{Porous Silicon}

\section{Silicon Light Emission}

Radiative recombination of an electron with a hole across the bandgap of semiconductors produces luminescence. The emitted photons have an energy equal to the bandgap energy (e.g., 1.4 to $1.5 \mathrm{eV}$ in $\mathrm{GaAs}$ ) and negligible momentum. Thus, the electron and the hole must be located at the same point in the Brillouin zone, which is the case in direct-gap semiconductors such as GaAs. Under these conditions, the radiative recombination rate is large, which means that the radiative lifetime is short (typically of the order of a few nanoseconds). To obtain a large luminescence efficiency, the nonradiative recombination rate must be lower than the radiative recombination rate. The efficiency is defined as

$$
\eta=\tau_{\text {nonrad }} /\left(\tau_{\text {nonrad }}+\tau_{\text {rad }}\right)
$$

where $\eta$ is the quantum efficiency, $\tau_{\text {rad }}$ is the radiative lifetime, and $\tau_{\text {nonrad }}$ is the nonradiative lifetime. Nonradiative recombination occurs both at the surface and in the bulk. To minimize it, the surface should be well passivated leading to a low surface recombination velocity $S$, and the bulk must be free of defects that act as radiation killers. The efficiency of good-quality, direct-gap III-V semiconductors exceeds $1 \%$ at room temperature and $10 \%$ at cryogenic temperature.

Because silicon is an indirect-gap semiconductor, electrons and holes are found at different locations in the Brillouin zone and recombination by emission of a photon alone is not possible. Photon emission is possible only if another particle capable of carrying a large momentum, such as a phonon, is involved. In this case, both energy and momentum can be conserved in the radiative transition. The participation of a third particle in addition to the electron and the hole makes the rate of the process substantially lower and the radiative lifetime typically in the millisecond regime. Thus, the efficiency drops by several orders of magnitude, even in the case of highpurity materials and good surface passivation. At room temperature, the typical efficiency of crystalline silicon is of the order of $10^{-5} \%$, which makes it unsuitable for LED's. ${ }^{\prime}$ Several attempts have been made to improve the luminescence efficiency of silicon and silicon-based alloys, including the use of silicon-germanium alloys and superlattices, ${ }^{2}$ isoelectronic impurities ${ }^{3}$ in a manner similar to nitrogen in $\mathrm{GaP}$, and erbium doping. ${ }^{4}$ The strategy in these attempts can be divided into two classes: increasing the radiative rate or decreasing the nonradiative rate. The former can be achieved by "bandgap engineering" or "defect engineering," which essentially consists of "convincing" the electron and the hole that they do not 
require a phonon to recombine radiatively. This process can be accomplished through zone folding in thin superlattices or with isoelectronic impurities whose energy level extends throughout the Brillouin zone. The latter consists of confining the electron and the hole to a small volume where the probability of finding a nonradiative center is equal to zero. This can be accomplished in the Ge-rich regions of SiGe and is realized to some extent with isoelectronic impurities. Such approaches have led to very good photoluminescence efficiencies (up to $10 \%$ in some cases) and good electroluminescence efficiency in some structures (approaching 1\%), but only at cryogenic temperatures. At room temperature, these numbers drop to the range of $10^{-4} \%$ to $10^{-5} \%$, and it is not clear how they can be increased to the $0.1 \%$ to $1 \%$ level.

\section{Porous Silicon}

Porous silicon is a material that has been known for nearly 40 years. ${ }^{5}$ It has found limited use in microelectronics, ${ }^{6}$ especially in the silicon-on-insulator (SOI) technology, as porous silicon becomes a good insulator after oxidation. In the 1980's, several studies of the optical properties of porous silicon were published, and photoluminescence in the deep red/near infrared was detected at cryogenic temperatures. ${ }^{7}$ In 1990, Canham $^{8}$ reported that when porous silicon is further etched in concentrated aqueous HF for $6 \mathrm{~h}$ after preparation, it emits bright red light when illuminated with blue or UV light. Longer etching in HF was demonstrated to lead to brighter photoluminescence at shorter wavelengths, which was ascribed to quantum confinement. Longer etching increases the porosity, which produces, on the average, smaller nanocrystalline columnar structures. When the dimension of the columns decreases below $5 \mathrm{~nm}$, the bandgap widens by quantum confinement in the conduction and valence bands and thus smaller columns produce larger bandgaps. In Canham's original model, the luminescence was attributed to band-to-band recombination across the bandgap. He also suggested that porous silicon might have a direct bandgap, which would explain the increase in efficiency by several orders of magnitude. Interestingly, at the same time as Canham was publishing his seminal paper, Lehmann and Gosele ${ }^{9}$ independently showed that the bandgap of porous silicon is larger than that of crystalline silicon and attributed this increase to quantum confinement as well.

Lehmann and Gosele's proposal allowed them to provide a new and elegant explanation for the mechanism of pore formation. Injection of holes from the substrate to the surface is required for etching in HF. In their model, the thinning of the silicon columns formed when the pores are created continues until quantum confinement produces a barrier against hole transport into the columns. Although there are attractive competing models, ${ }^{10}$ which for example rely on electrostatic effects near the pore's tip, this model has received partial experimental confirmation. ${ }^{11}$ It must be noted that neither Canham's model for the luminescence nor Lehmann and Gosele's model for pore formation is universally accepted, and other models have been proposed. ${ }^{12-16}$ Note also that the nanostructure of porous silicon is quite complicated. Even though columnar crystalline structures are present in some samples, the shape, thickness, and orientation of these columns or wires are not uniform. In other samples, no wires are detected, and the crystalline objects appear to be more or less spherical (“dots"). In all cases, however, light emission appears to be well correlated with the presence of crystalline structures smaller than $5 \mathrm{~nm} .{ }^{17}$

Figure 62.36 shows the schematic arrangement for a typical electrochemical cell used to produce porous silicon. The arrangement is very simple and inexpensive. The wafer (anode) and the metal cathode are immersed in an aqueous solution containing HF. Typically, the HF concentration is kept around $25 \%$ by weight, and ethanol or methanol is added to improve the penetration of the solution into the pores and to minimize hydrogen bubble formation. Maintaining a constant current density of 1 to $100 \mathrm{~mA} / \mathrm{cm}^{2}$ for several minutes results in the formation of a porous silicon film with a thickness ranging from $\sim 1 \mu \mathrm{m}$ to tens of microns. Both the thickness and the photoluminescence spectrum of the porous layer depend

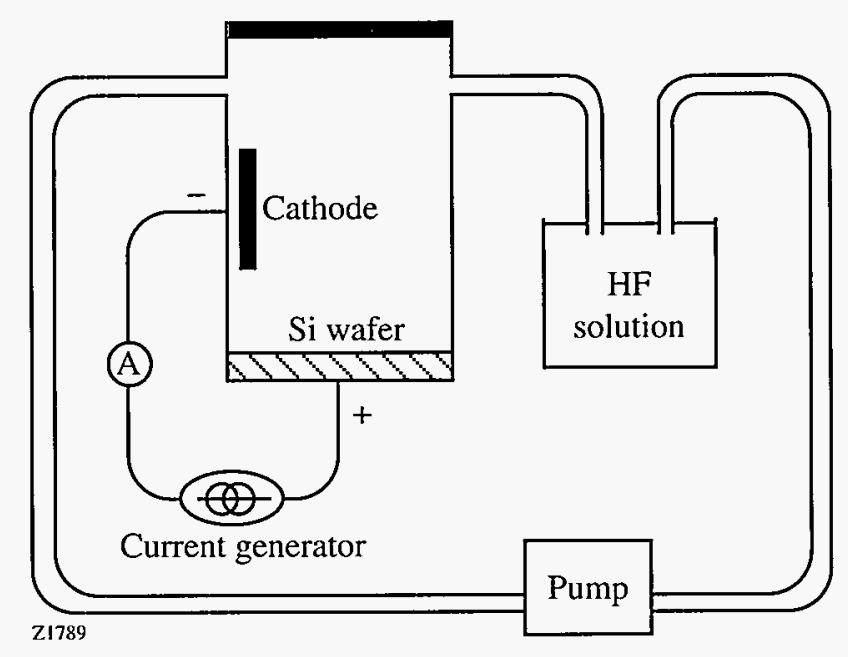

Figure 62.36

Schematic of the electrochemical cell used for the production of porous silicon. The HF solution circulates in Teflon ${ }^{\mathrm{TM}}$ tubing and through a Teflon ${ }^{\mathrm{TM}}$ pump. 
strongly on many parameters, such as doping level and sign, current density, solution concentration and $\mathrm{pH}$, and duration of the anodization. However, it should be stressed that lightemitting porous silicon can be produced over a wide range of most of these parameters. For example, holes, which play a critical role in the formation of porous silicon, cannot be injected from the bulk to the surface of an $n$-type wafer. This shortcoming can be eliminated by illuminating the wafer during anodization, as holes are photogenerated near the surface. Even if the porous layer is not of the luminescent type, further etching in HF in the open circuit configuration or by anodic oxidation in water can produce bright luminescence.

Figure 62.37 shows a LEPSi luminescence spectrum and its time evolution after pulsed excitation at $3.5 \mathrm{eV}$. The peak photoluminescence wavelength is typical, but it can easily be tuned between $850 \mathrm{~nm}$ and $550 \mathrm{~nm}$, as discussed later. The fullwidth at half-maximum (FWHM) of the spectrum is between $150 \mathrm{meV}$ and $500 \mathrm{meV}$. The most widely accepted explanation for this very large width is that the spectrum is "inhomogeneous," i.e., corresponds to bandgap or near-bandgap emission coming from nanocrystallites with a distribution of sizes. The decay of the photoluminescence following excitation takes place on a microsecond time scale at room temperature and increases to the millisecond regime at cryogenic temperatures. ${ }^{18}$ Furthermore, the short-wavelength components usually decay faster than the long-wavelength components, a fact that

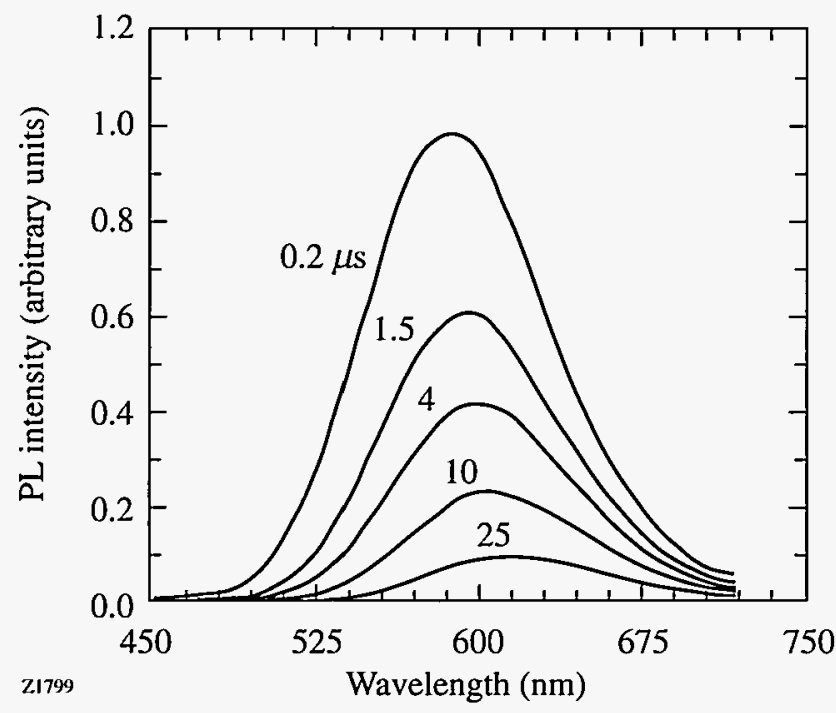

Figure 62.37

Time-resolved photoluminescence spectra measured at different times following excitation with a picosecond laser pulse at $3.5 \mathrm{eV}$. The overall decay at room temperature is in the microsecond time domain, but the decay is not exponential and is faster at shorter wavelengths. also supports the hypothesis that a distribution of crystallite sizes produces the wide spectrum. This spectral diffusion with time varies from sample to sample. The decay of the full band or that of any individual wavelength component does not follow a simple law and can be fitted very well using a stretched exponential function. ${ }^{19}$ Stretched exponentials may result from the random walk of carriers on a finite distribution of discrete sites in real space, as is known to occur in amorphous silicon. Regardless of the details of the physical mechanisms, there are well-known procedures to define the average or most probable lifetime; this lifetime value is usually quoted in the literature. After removing a possible fast decay component in the nanosecond time domain (to be discussed later), the lifetime of the red luminescence is in the microsecond time domain.

\section{The Three Luminescence Bands}

\section{The Red Band}

As mentioned earlier, the "red" luminescence band actually extends from the near infrared to the orange/green regions. Tuning can be achieved by changing any one of many parameters. ${ }^{20}$ Here, we focus on results obtained by changing the anodization time under light assistance and by annealing in air. The LEPSi samples were prepared from $p$-type $S i$ substrates in the dark (DA) or from $p$ - and $n$-type Si substrates with light assistance (LA). The substrates were (100) oriented with a resistivity in the 1- to $10-\Omega-\mathrm{cm}$ range. Anodization with LA was performed using a $300-\mathrm{W}$, white-light halogen lamp.

The photoluminescence (PL) spectra from DA and LA samples are rather different. ${ }^{21}$ The DA LEPSi has a broad peak centered near $740 \mathrm{~nm}$, which is typical of samples prepared in the dark. In contrast, the PL spectra of the LA samples can be easily tuned from the infrared to the yellow/orange by increasing the anodization time. For a given solution and a constant current density, a longer anodization time produces a thicker LEPSi layer and shifts the PL peak toward shorter wavelengths. Figure 62.38 shows the relation between PL intensity, PL peak wavelength, and anodization time for $n$-type Si wafers prepared in an aqueous solution (no alcohol) with LA using a current density $j$ of $10 \mathrm{~mA} / \mathrm{cm}^{2}{ }^{22}$ Under these conditions, the maximum PL intensity corresponds to a peak wavelength near $700 \mathrm{~nm}$. A proper choice of solutions and current density allows us to obtain homogeneous LEPSi samples that have good PL efficiency. Using blue excitation, the highest quantum efficiency we have measured on a red LEPSi sample at room temperature is approximately $5 \%$. After optimization of the growth conditions, the PL of LEPSi samples is comparable to that emitted at room temperature by direct-gap III-V semiconductor compounds. 


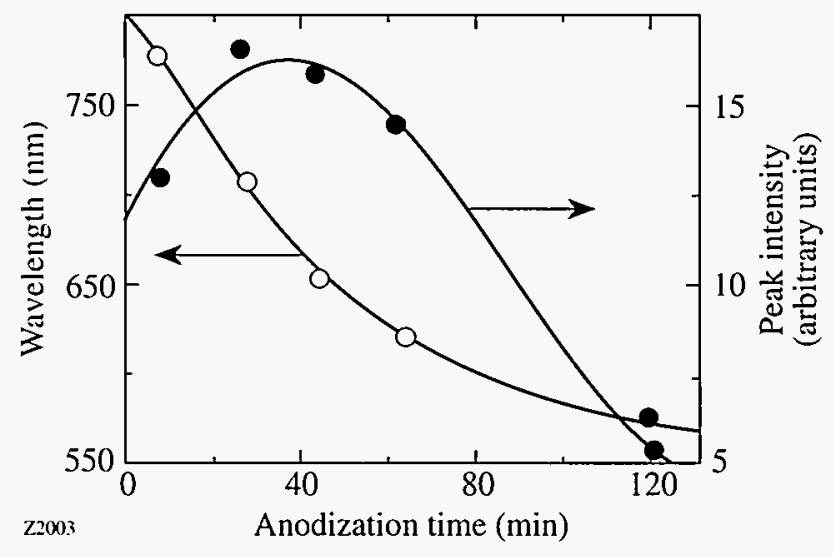

Figure 62.38

Photoluminescence intensity and peak wavelength as a function of the anodization time. The current density is $10 \mathrm{~mA} / \mathrm{cm}^{2}$, the substrate is a 10- $\Omega$-cm $n$-type Si wafer, and light assistance is employed.

The PL peak wavelength has also been tuned by thermal annealing in a tube furnace in ambient air for $5 \mathrm{~min}$ at various temperatures. ${ }^{23}$ In this case, the LEPSi layers were formed by anodization in the dark of $10-\Omega$-cm $p$-type Si wafers in a $1: 1: 2$ solution of $\mathrm{HF}, \mathrm{H}_{2} \mathrm{O}$, and methanol. Figure 62.39 summarizes results obtained with a variety of samples, either freshly prepared, annealed in air, or oxidized in $\mathrm{HNO}_{3}$. The PL peak wavelength shifts to shorter values as the ratio of $\mathrm{Si}-\mathrm{O}$ bonds to $\mathrm{Si}-\mathrm{H}$ bonds increases. The ratio of the concentration of $\mathrm{Si}$ $\mathrm{H}$ and $\mathrm{Si}-\mathrm{O}$ bonds present on the surface of the nanocrystallites as shown in Fig. 62.39 is not the absolute ratio, since the cross section for infrared absorption of $\mathrm{Si}-\mathrm{O}$ bonds is stronger than that for the Si-H bonds. Thus, the horizontal scale in Fig. 62.39 is a relative scale only. These results demonstrate a clear correlation between surface chemistry and PL peak wavelength. Freshly anodized samples that contain a very small but detectable amount of $\mathrm{Si}-\mathrm{O}$ bonds and chemically oxidized samples that have almost no remaining $\mathrm{Si}-\mathrm{H}$ bonds fit nicely at the two extremes of the data. Furthermore, annealing of freshly prepared samples at moderate temperature leads to data points that coincide with the data points obtained with aged samples.

The results of Fig. 62.39 are more easily understood if we assume that the PL is related to recombination involving surface states ${ }^{24}$ rather than if it is strictly due to confinement ${ }^{8}$ since the low-temperature treatments reported here have no effect on the particle size. For a particular surface coverage of the nanocrystallites present in LEPSi, we expect to find a specific set of surface states. When the coverage is changed, the nature and the energetic position of the surface states within the bandgap should change; thus, the PL spectrum is expected to change as well. It may be reasonable to model the wide PL spectrum as due to several bands associated with recombination involving different surface species.

It is worth noting that the origin of the "red" luminescence remains highly controversial. Many models have been proposed, ranging from those involving specific defects and molecular compounds to those involving quantum confinement. $^{12-16,25}$ Of all these models, two have received strong experimental support. The "pure" quantum confinement originally proposed by Canham, ${ }^{8}$ which is consistent with many observations, has recently received support from resonant photoluminescence experiments. ${ }^{26,27}$ In these experiments the low-temperature luminescence was not excited by a shortwavelength laser but by a laser tuned inside the broad PL spectrum to provide size-selective excitation. Under these conditions, the PL spectrum shows discrete steps that coincide with the zone-edge phonons of crystalline silicon that are known to be involved in the bandgap absorption and emission in crystalline silicon. These results not only indicate that silicon is the absorbing and luminescing species in LEPSi but also strongly suggest that luminescence occurs at the silicon crystallite bandgap. Other experiments, however, suggest that the luminescence occurs well below the bandgap and is affected by more than quantum confinement. Some examples include the results shown in Fig. 62.39 and the difference between the measured PL peaks and the measured or calculated bandgap, ${ }^{28,29}$ which can be as large as $\sim 1 \mathrm{eV}$.

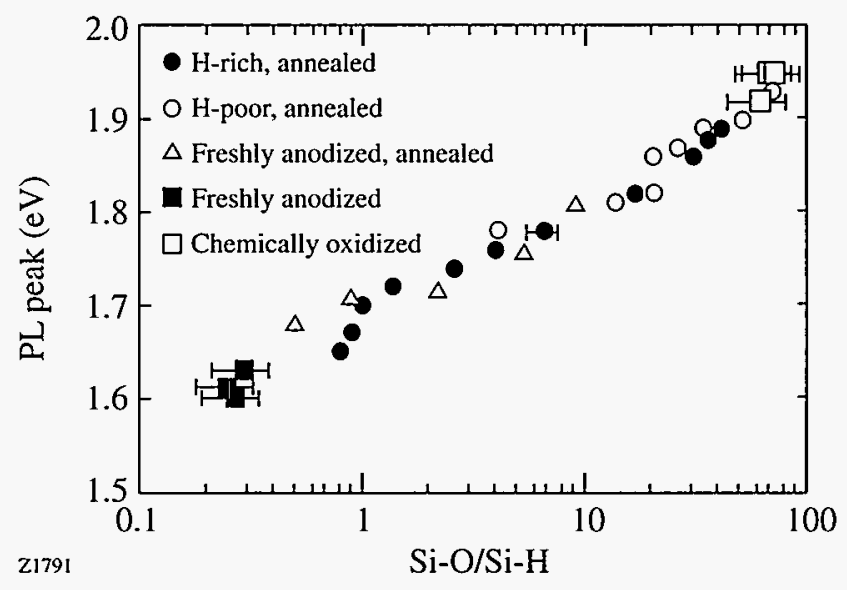

Figure 62.39

Relationship between the peak of the photoluminescence spectrum and the surface coverage, as measured by the ratio of $\mathrm{Si}-\mathrm{O}$ bonds over $\mathrm{Si}-\mathrm{H}$ bonds obtained by infrared absorption measurements. This ratio does not take into account the difference in the infrared absorption cross sections between $\mathrm{Si}-\mathrm{H}$ and $\mathrm{Si}-\mathrm{O}$ bonds. 
Since the PL intensity and spectrum depend strongly on the surface coverage, one can expect some problems with longterm stability of the PL. For hydrogen-covered surfaces, the surface can become depassivated by hydrogen loss through heating ${ }^{30}$ or exposure to short-wavelength light. ${ }^{31} \mathrm{~A}$ solution to this problem is passivation with silicon dioxide instead of hydrogen. Such samples are much more resilient ${ }^{21}$ but they also tend to luminesce in the blue.

\section{The Blue Band}

Recent literature contains several reports of strong blue PL in LEPSi after rapid thermal oxidation (RTO) at high temperature $\left(T_{\mathrm{ox}} \geq 1000^{\circ} \mathrm{C}\right),{ }^{32,33}$ as well as several reports of weaker blue PLin various samples. We conducted a systematic investigation of the properties of blue samples and have evaluated methods for obtaining them. The LEPSi layers were formed by anodization of $5-\Omega-\mathrm{cm} p$-type silicon wafers in a 1:1:2 solution of $\mathrm{HF}, \mathrm{H}_{2} \mathrm{O}$, and methanol. To change the sample's properties we varied the current density from $1 \mathrm{~mA} /$ $\mathrm{cm}^{2}$ to $30 \mathrm{~mA} / \mathrm{cm}^{2}$. Anodization was performed either in the dark or with light assistance. Oxidation of the LEPSi layers was achieved in a tube furnace with a dry oxygen atmosphere. Samples of LEPSi first anodized in the dark at a current density $j=10 \mathrm{~mA} / \mathrm{cm}^{2}$ and then oxidized for $2 \mathrm{~min}$ at $1100^{\circ} \mathrm{C}$ in dry oxygen $^{34}$ display the reported blue PL band (Fig. 62.40). Infrared absorption measurements show a strong line at $1080 \mathrm{~cm}^{-1}$ related to the asymmetric stretching vibrational mode of the Si-O-Si oxygen bridge (Fig. 62.40, inset). Other LEPSi samples anodized at a smaller current density $\left(1 \mathrm{~mA} / \mathrm{cm}^{2}\right)$ with light assistance and subjected to no further treatments have only one strong peak at $1070 \mathrm{~cm}^{-1}$ in the infrared spectrum, which corresponds to Si-O-Si bridges in a configuration close to stoichiometry. Thus, the infrared spectra show no significant difference in the chemical composition between these two types of samples. Although we refer to these samples as "fully oxidized" in the following discussion since no $\mathrm{Si}-\mathrm{H}$ bonds remain, this does not imply that there are no silicon nanocrystallites left in these samples.

The time-resolved PL spectra of both samples show a broad PL band (FWHM $>0.5 \mathrm{eV}$ ) with a peak near $2.6 \mathrm{eV}$ that decays on a nanosecond time scale. The PL spectra have not been corrected for the spectral sensitivity of our detection system and are likely to extend to shorter wavelengths. The decay is nonexponential as for the red band, but in contrast to the red band, no significant wavelength dependence of the blue PL decay has been observed when the detection wavelength was changed from $440 \mathrm{~nm}$ to $650 \mathrm{~nm}$, unlike what is seen in the red PL. The decay dynamics did not change appreciably when the measurements were performed at cryogenic temperatures. The observed nonexponential decay may indicate a distribution of carrier lifetimes, with the most probable lifetime being close to $1 \mathrm{~ns}$. The estimated quantum efficiency of the blue PL is greater than $0.1 \%$, roughly one order of magnitude below the efficiency of a good red sample.

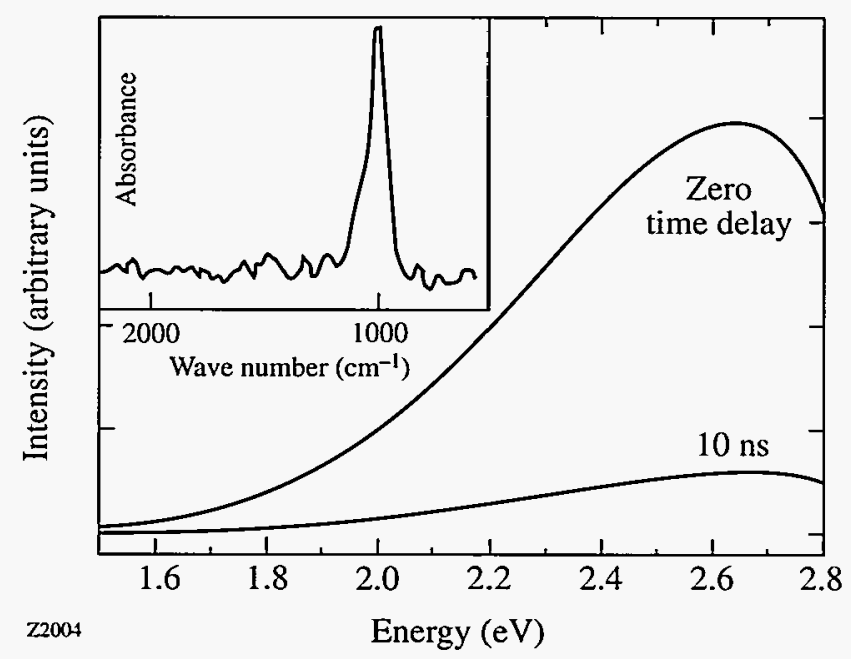

Figure 62.40

Time-resolved photoluminescence spectra of oxidized LEPSi after excitation with a picosecond laser pulse at $3.5 \mathrm{eV}$. The infrared absorption spectrum of the same sample shown in the inset indicates that the sample contains no $\mathrm{Si}-\mathrm{H}$ bonds and only $\mathrm{Si}-\mathrm{O}$ bonds.

The blue PL is also observed in samples that are oxidized chemically by immersion in $10 \% \mathrm{HNO}_{3}$ for 2 min or in freshly anodized samples after handling in air. ${ }^{34}$ Measurements of the blue PL dynamics in the chemically oxidized sample also show a nonexponential decay with a characteristic time close to those of the fully oxidized samples. The time-resolved PL spectra of the freshly anodized samples measured right after excitation again show a weak but clearly observable blue PL band whose spectrum is similar to that of the "fully oxidized" samples. The presence of a very small amount of Si-O bonds is apparent in the infrared spectra of freshly anodized samples. Due to the low intensity of this blue band we were not able to analyze the decay in detail, but the characteristic decay time is much less than $15 \mathrm{~ns}$.

Our results suggest two possibilities for the origin of the blue PL. Silicon dioxide is known to luminesce efficiently in the visible under appropriate conditions, ${ }^{35}$ and it has been shown that blue PL with a nanosecond decay time can be excited by $4-\mathrm{eV}$ photons in high-purity, wet synthetic silica. ${ }^{36}$ Thus, the blue PL in oxidized LEPSi could originate not from 
the silicon crystallites themselves, but from the oxide surrounding them. The second possibility is that special Si-O configurations produce the luminescence. These special configurations could be present at the Si nanocrystal/silicon dioxide interface, or in Si-rich regions produced in the oxide after nearly complete oxidation of the crystallites. It appears that we can exclude the hypothesis that the blue PL would originate from the interior of sub-3-nm Si crystallites. If this were the case, subjecting the oxidized crystallites to chemical agents should not affect the luminescence. We have exposed blueemitting oxidized porous silicon to liquid methanol and observed a quenching of the blue PL. ${ }^{37}$ This quenching is consistent with luminescent centers near the oxide surface. Specific chemical configurations such as silanol ${ }^{38}$ have been proposed, but there remains considerable uncertainty as to the exact origin of the blue PL. ${ }^{39}$ Note finally that the stability of the blue samples in air is much improved compared to that of the red samples. ${ }^{21}$ This improvement can be traced to the presence of silicon dioxide, which is much less fragile than the $\mathrm{Si}-\mathrm{H}$ bonds that passivate the surface of freshly prepared LEPSi.

\section{The Infrared Band}

Very few reports of infrared PL have appeared in the literature. ${ }^{8.24 .40-42}$ Some of these measurements were interpreted as bandgap or near-bandgap PL from crystalline silicon. Another explanation has been proposed for this IR PL, in which emission is associated with mid-gap dangling bonds on the surface of the nanocrystallites. ${ }^{24}$ We have performed a careful measurement of the IR PL in several LEPSi samples. ${ }^{41}$ In our experiments, the samples were placed in an ultrahigh vacuum (UHV) chamber where they were annealed in situ. The 422-nm line of a HeCd laser excites the PL, which in turn is detected by a Ge detector with a cutoff of $\sim 0.7 \mathrm{eV}$. In the results reported below, the PL spectrum was recorded at room temperature, following vacuum annealing at temperatures as high as $\sim 500^{\circ} \mathrm{C}$ for $5 \mathrm{~min}$.

Figure 62.41 shows the room-temperature PL spectra for one sample before annealing and after annealing at two temperatures. The broad IR PL peak of the as-prepared sample is small but measurable at room temperature. As the annealing temperature is increased, its relative intensity increases until it dominates the spectrum. The IR PL appears to be most intense after annealing at a temperature where the red PL disappears, i.e.. when most of the hydrogen that passivates the LEPSi surface has been desorbed, since heat treatment at such a temperature produces many dangling bonds. It is thus tempting to interpret the IR PL as due to recombination involving carriers in mid-gap dangling bonds. We also prepared samples in which the IR PL peak energy was $\sim 0.7 \mathrm{eV}$. After annealing around $350^{\circ} \mathrm{C}$, the integrated PL intensity measured at room temperature was comparable to the integrated intensity of the initial red PL peak. Preliminary results obtained with a luminescence detection system that is sensitive to wavelengths up to $5 \mu \mathrm{m}$ indicate that these samples luminesce well past $2 \mu \mathrm{m}$. The conditions required for obtaining such samples are as of yet not well understood.

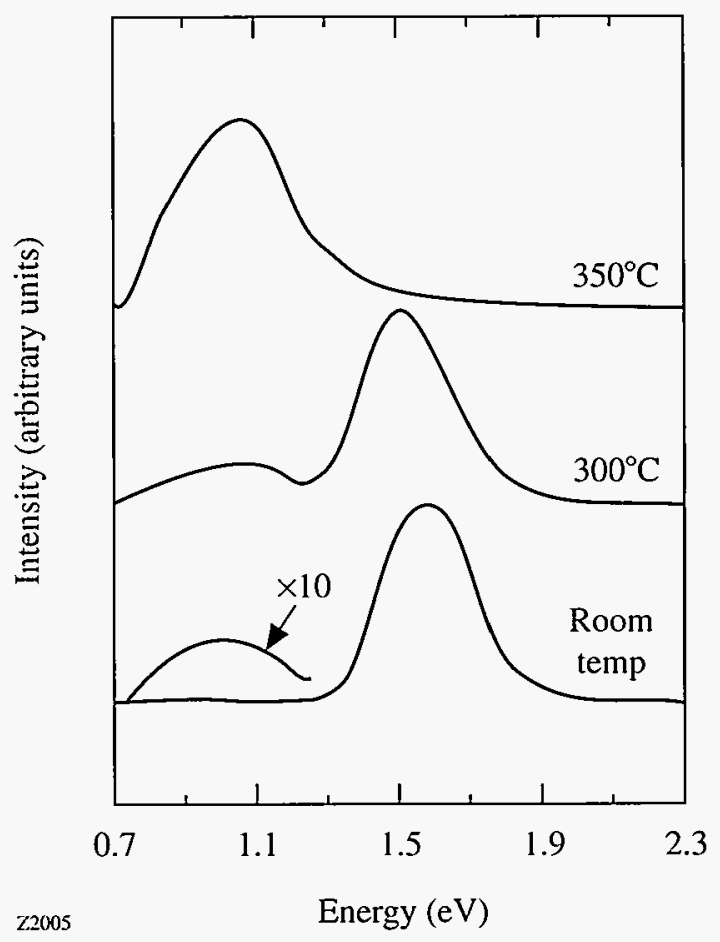

Figure 62.41

Normalized room-temperature PL spectra for LEPSi held in ultrahigh vacuum, before and after annealing for $5 \mathrm{~min}$ at two temperatures.

Time-resolved PL measurements ${ }^{42,43}$ indicate a decay in the range from tens of nanoseconds to $\sim 10 \mu \mathrm{s}$ at $77 \mathrm{~K}$, which is faster than the red PL decay in the same range of temperature. This decay may be consistent with calculations of the radiative recombination in the infrared due to dangling bonds present at nanocrystallite surfaces. ${ }^{44}$ Finally, the long-term stability of the infrared band has not been established. If it is related to the presence of dangling bonds, it is reasonable to expect poor stability since the concentration and nature of dangling bonds on the surface can be modified by many factors. Our preliminary results so far support this hypothesis.

\section{Femtosecond Photoinduced Absorption}

1. Experimental Techniques and Sample Preparation

The femtosecond time-resolved experiments have been 
performed with the standard pump/probe arrangement. The pump beam excites electrons and holes across the bandgap, while the probe beam monitors the changes in transmission (and reflection) induced in the sample by photoinjection of carriers by the pump beam. The pump and probe beams are synchronized since they are produced by the same laser pulse. Thus, the time evolution of the changes in transmission (and reflection) is monitored by increasing the beam path of the probe, which produces a time delay between the pump and probe beams. These experiments, which we routinely perform in semiconductors, ${ }^{45-47}$ have a time resolution limited by the pulse duration (in the present case, $100 \mathrm{fs}$ ). Once the results have been recorded, the changes in optical properties are related to the dynamics of the photoinjected carriers, which may include thermalization, cooling, trapping, and recombination. In cases where nonradiative recombination occurs rapidly across the bandgap, ${ }^{47,48}$ it is also possible to record the dynamics of the temperature increase of the sample due to the production of a large number of phonons.

Femtosecond photoinduced measurements were performed using two different laser systems. In the first type of experiments, ${ }^{49}$ the laser source was a colliding-pulse, modelocked dye laser, amplified at $8.5 \mathrm{kHz}$ using a copper vapor laser. The pulses are $<100 \mathrm{fs}$ long at $620 \mathrm{~nm}(2 \mathrm{eV})$. A portion of these pulses is used to generate a white light continuum from which 100 -fs-long pulses tunable from the green to $\sim 900 \mathrm{~nm}$ were selected. In the second type of experiments, ${ }^{50}$ the laser source was a titanium sapphire laser producing 90 -fs-long pulses tunable between 800 and $900 \mathrm{~nm}(1.55$ to $1.38 \mathrm{eV})$ with a repetition rate of $85 \mathrm{MHz}$. Pulses at half the wavelength (twice the photon energy) were obtained by frequency doubling in a BBO nonlinear crystal.

To perform the transmission measurements, the samples must first be removed from their silicon substrates, as the substrates would absorb all the light at wavelengths shorter than $1 \mu \mathrm{m}$. In the present work, we used both oxidized mesoporous films ${ }^{49}$ and very-high-quality films deposited on sapphire. ${ }^{50,51}$ These latter films were produced using a current density of $14 \mathrm{~mA} / \mathrm{cm}^{2}$ passing through a circular area of $1.76 \mathrm{~cm}^{2}$ from $25 \mathrm{~s}$ to 30 min depending on the desired thickness. The anodization occurred in the dark and the (100) polished c-Si wafers were either moderately or highly doped. To remove the porous silicon film from the substrate, electropolishing was employed in which the silicon atoms were removed layer by layer and the porous silicon film was separated from the wafer (lifted off). The process was converted from porous silicon formation to electropolishing by diluting the HF while increasing the current density to approximately $100 \mathrm{~mA} / \mathrm{cm}^{2}$. The freestanding LEPSi films were kept wet in ethanol to protect them from breaking during drying, and layers thinner than $\sim 5 \mu \mathrm{m}$ were deposited wet on sapphire windows and allowed to dry in air. Afterward, the LEPSi films remained attached to the windows by van der Waals or electrostatic forces, similar to a technique used to mount ultrathin III-V semiconductor films. ${ }^{45}$ We chose sapphire windows because of their optical flatness, high heat conductivity, and high transparency from the IR to the UV. Figure 62.42 summarizes some of the properties of a typical high-quality ultrathin LEPSi film.

\section{Experimental Results}

Figure 62.43 shows results obtained with the pump at $2 \mathrm{eV}$ (which is well above the PL peak of $1.65 \mathrm{eV}$ ) and various probes at photon energies ranging from above to below the PL peak energy. ${ }^{49}$ Qualitatively similar results have been obtained on all films, irrespective of whether they were pumped at 2 or $3 \mathrm{eV}$, in the entire probe wavelength range. These traces have several interesting features:

- At all probe wavelengths, the absorption increases instantly, then recovers in part very promptly to a photoinduced absorption "plateau."

- When the induced absorption is measured on a much longer time scale, this "plateau" corresponds in fact to a slow recovery of the photoinduced absorption.

- The recovery is nonexponential and depends on the photoinjected carrier density: at low injected carrier density, the prompt recovery is extremely fast and the slower recovery takes tens of picoseconds, whereas at high injected carrier density, the prompt recovery slows down and the slower recovery speeds up.

The challenge is to interpret these results.

Our results indicate that porous silicon is not a direct-gap semiconductor. In direct-gap semiconductors, bleaching is observed after femtosecond excitation. ${ }^{45,46,52}$ This bleaching results from the partial occupation of the final and initial states by the photoinjected electrons and holes respectively, which decreases the absorption at all probe wavelengths and especially near the pump wavelength immediately after excitation. In the present experiments, we instead observe photoinduced absorption, which occurs either in indirect-gap semiconductors ${ }^{53,54}$ or in amorphous semiconductors, ${ }^{48,55}$ and is indicative 


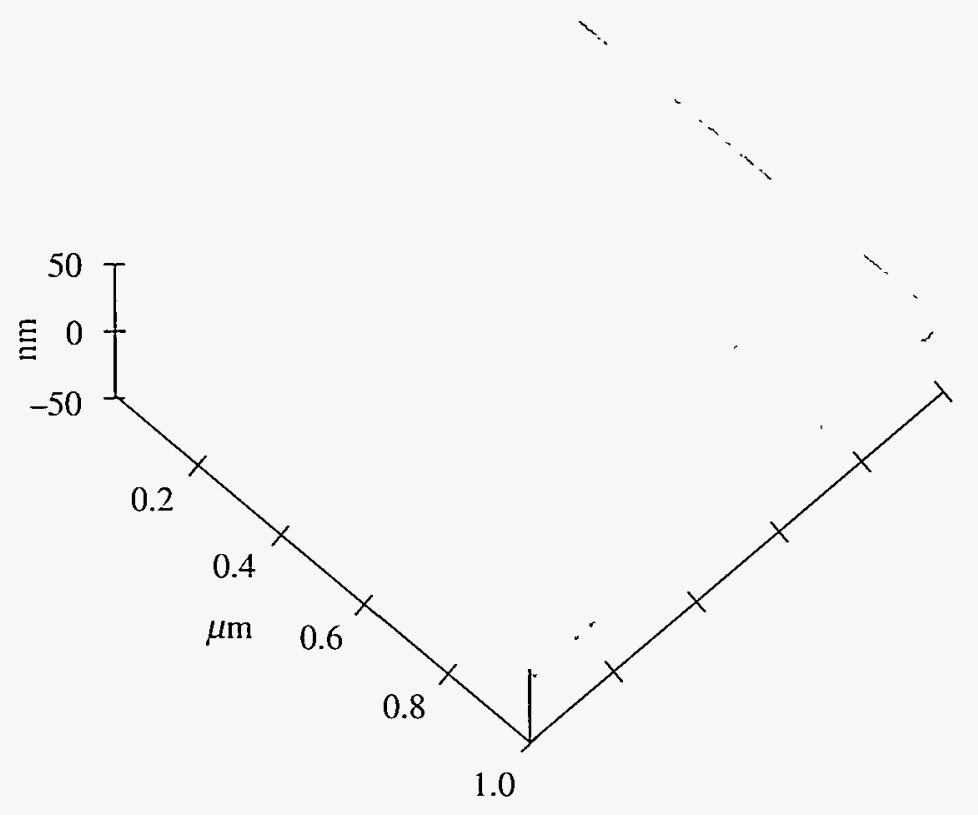

21828
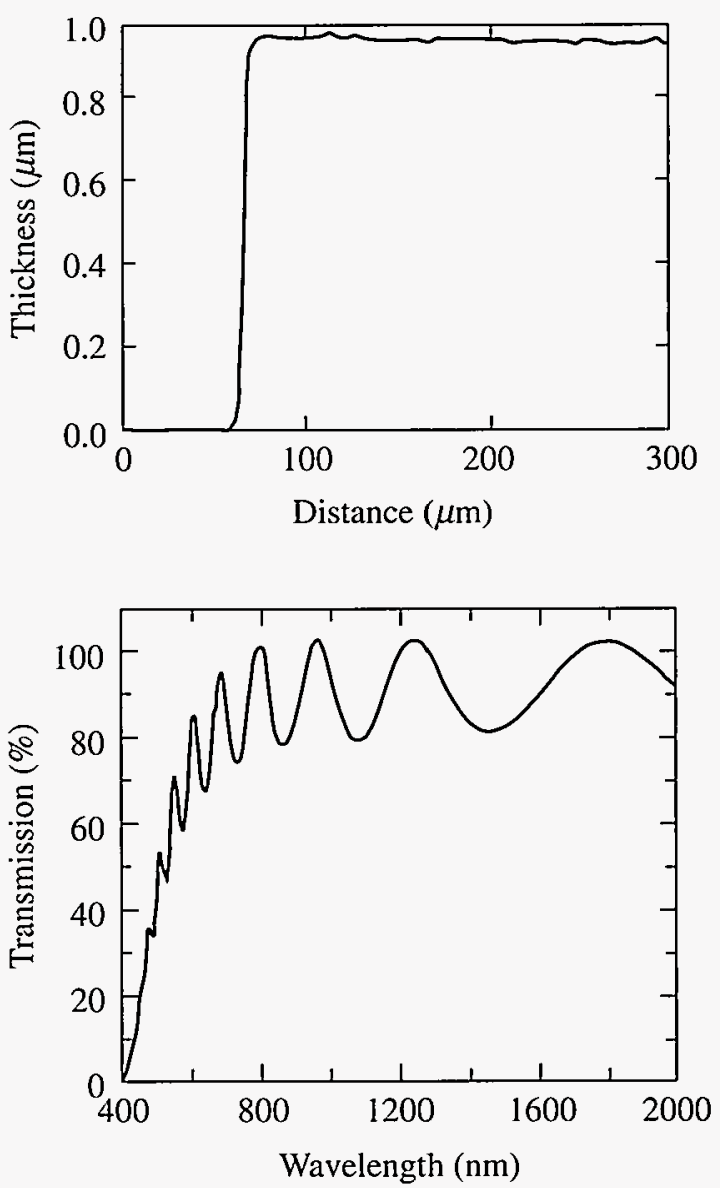

Figure 62.42

Atomic-force-micrograph, thickness measurement, and optical transmission spectrum of a thin, porous film that has been lifted off the c-Si substrate and deposited on a sapphire substrate.

of Drude absorption by free carriers. This observation confirms recent theoretical ${ }^{56}$ and experimental ${ }^{26,27}$ work, which also suggested that red-emitting porous silicon remains an indirect bandgap semiconductor. Figure 62.44 compares femtosecond time-resolved data obtained under similar conditions with GaAs (a direct-gap semiconductor), a-Si:H (an amorphous semiconductor), c-Si (an indirect-gap semiconductor), and porous silicon. Clearly, porous silicon and $\mathrm{c}-\mathrm{Si}$ have a very similar response, which in turn is quite distinct from that of GaAs and a-Si:H.

The dynamics of the photoinduced absorption recovery are consistent with the following model. The prompt recovery is due to carrier trapping, presumably at the nanocrystallite surfaces. The fact that trapping occurs on a $\sim 100$-fs time scale should not be surprising since a classical electron moving at the thermal velocity will "hit" the surface every 30 fs. However, not all nanocrystals have a "fast" trap, and the ratio of the amplitude of the slower recovery to that of the prompt recovery is a measure of the ratio of the number of nanocrystals without a fast trap to that of nanocrystals with a fast trap. In those nanocrystals that have no fast trap, the photoinjected carriers can only recombine or be trapped in "slow" traps. Assuming that the slower recovery is due to recombination, we can explain the intensity dependence. When the number of electron-hole pairs injected per nanocrystallite is less than 1, recombination is probably radiative and relatively slow; when the number of pairs injected exceeds 1 per nanocrystal, Auger recombination becomes possible. In this process, one electron recombines with one hole, and the excess energy is given to another electron (or hole). This process is relatively efficient at large carrier densities in bulk crystalline silicon and is expected to be very efficient in nanometer-size crystallites, where the overlap between the wave functions of the carriers is strong and the momentum conservation rules that limit the Auger rate in bulk silicon are somewhat relaxed. Furthermore, the 


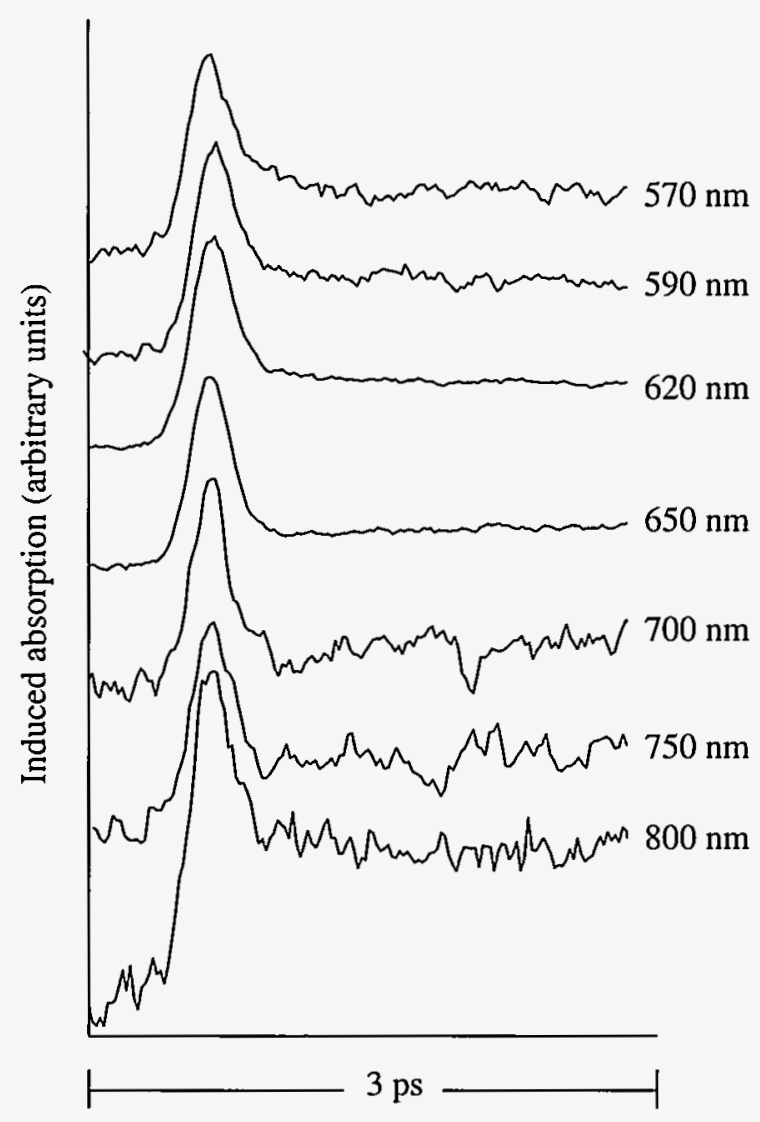

21766

Figure 62.43

Induced absorption measured with a probe pulse at different wavelengths following carrier injection with a $100-\mathrm{fs}-\mathrm{long}, 2-\mathrm{eV}$ pump pulse in a sample that luminesces near $1.65 \mathrm{eV}$. The traces are similar at all wavelengths.

relative amplitude of the prompt recovery is expected to decrease since in nanocrystallites with one trap the second photoinjected pair can no longer undergo fast trapping.

We thus attribute the initial recovery to trapping of the photoexcited carriers from extended states into surface states. The intensity dependence of the fast recovery is consistent with trap saturation. These traps are most likely not responsible for the luminescence because trapping tends to become more efficient when the luminescence efficiency decreases after exposure to high-intensity UV light. However, the changes in the ultrafast optical response remain small even after the luminescence efficiency drops by at least one order of magnitude.

\section{Electroluminescent Devices}

Electroluminescence (EL) was observed in LEPSi shortly after the discovery of its strong photoluminescence. ${ }^{57-61}$ It was first detected during anodic oxidation in the electrochemi-

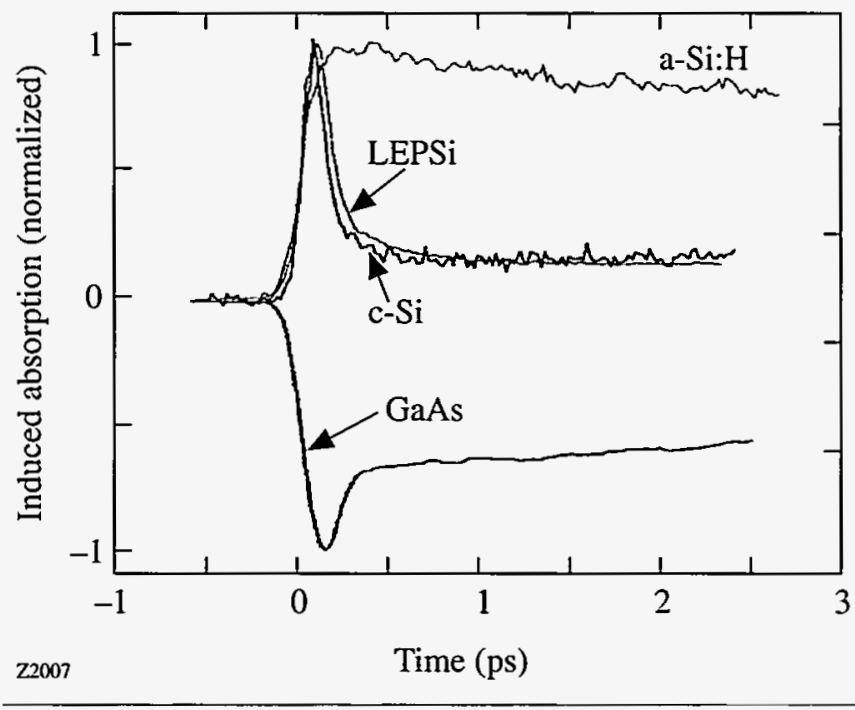

Figure 62.44

Room-temperature photoinduced absorption measurements performed in the pump-probe geometry at $2 \mathrm{eV}$ in GaAs, crystalline silicon (c-Si), porous silicon (LEPSi), and amorphous hydrogenated silicon (a-Si:H).

cal cell. ${ }^{61}$ This EL and electrolyte electroluminescence were shown to have a spectrum similar to the PL spectrum, with an efficiency larger than $0.1 \%$. Bsiesy et al. ${ }^{62}$ reported on tuning the electrolyte EL throughout the broad PL spectrum by simply changing the applied voltage from $0.8 \mathrm{~V}$ to $1.5 \mathrm{~V} .{ }^{62}$ Cathodoluminescence has also been reported. ${ }^{63,64}$ In this section, we focus on solid-state electroluminescent devices. In these light-emitting devices (LED's), electrons and/or holes are injected into the porous layer where radiative recombination occurs.

The first LED made of porous silicon was demonstrated in $1991 .{ }^{57}$ It consisted of a metal/porous silicon/crystalline silicon structure similar to a Schottky barrier or more accurately to a metal/insulator/semiconductor (MIS) structure. Although the device did electroluminesce, its efficiency measured at room temperature was of the order of $10^{-4} \%$ to $10^{-5} \%$. The threshold voltage for observable EL was at first very high $(\gg 10 \mathrm{~V}$ ) but has since been reduced to less than $5 \mathrm{~V}$. In our recent work, we have been able to detect EL for applied voltages below $1 \mathrm{eV}$ in more complicated structures. ${ }^{65}$ Such LEPSi MIS devices are very easy to fabricate since after fabrication of the porous layer, the only additional step is the evaporation of a thin metal film. Variations on this device structure have since been published by many groups. The PL and EL spectra are usually similar to each other, although in some cases not identical ${ }^{66,67}$ for reasons that are not clear. The efficiency of these devices has remained low, although efficiencies in the range of $10^{-2} \%$ and even higher have been 
quoted. ${ }^{68.69}$ Some of the most obvious reasons for the poor efficiency are (1) the mechanism of carrier injection is not clear and possibly inefficient; (2) the semitransparent metal contacts absorb part of the luminescence; and (3) it is extremely difficult to make a good contact over the large microscopic surface area of porous silicon using line-of-sight deposition techniques. In other LED's indium tin oxide (ITO) is used as the top electrode ${ }^{58}$ instead of a metal. This approach has several advantages, including potentially better contacts and less light absorption by the ITO. We have found in our devices that the efficiency of LED's using ITO is consistently higher than that of LED's using metals such as Au or Ag. Contacts made of conducting polymers have also been demonstrated ${ }^{70}$ but the efficiency remained low.

The best published efficiency for porous silicon LED's was achieved using a somewhat more complicated device structure. ${ }^{69}$ Figure 62.45 shows the proposed band structure and the current-voltage $(\mathrm{I}-\mathrm{V})$ relationship for a similar device. ${ }^{66,71}$ The porous silicon $p$ - $n$ junction LED's were fabricated in $p$ type substrates with a resistivity of 5 to $10 \Omega \mathrm{cm}$. A high-dose phosphorus implant was followed by a period of thermal annealing to yield junction depths $\leq 1 \mu \mathrm{m}$. Because holes are required in the anodization process, light assistance is essential to create $n$-type LEPSi. The LEPSi layers were thus formed by electrochemical etching in a $1: 1: 2$ solution of $\mathrm{HF}, \mathrm{H}_{2} \mathrm{O}$, and ethanol, using a constant current density of $15 \mathrm{~mA} / \mathrm{cm}^{2}$ for 5 to $30 \mathrm{~min}$. This anodization process produces LEPSi at a rate of approximately $1 \mu \mathrm{m} / \mathrm{min}$. To improve the homogeneity of

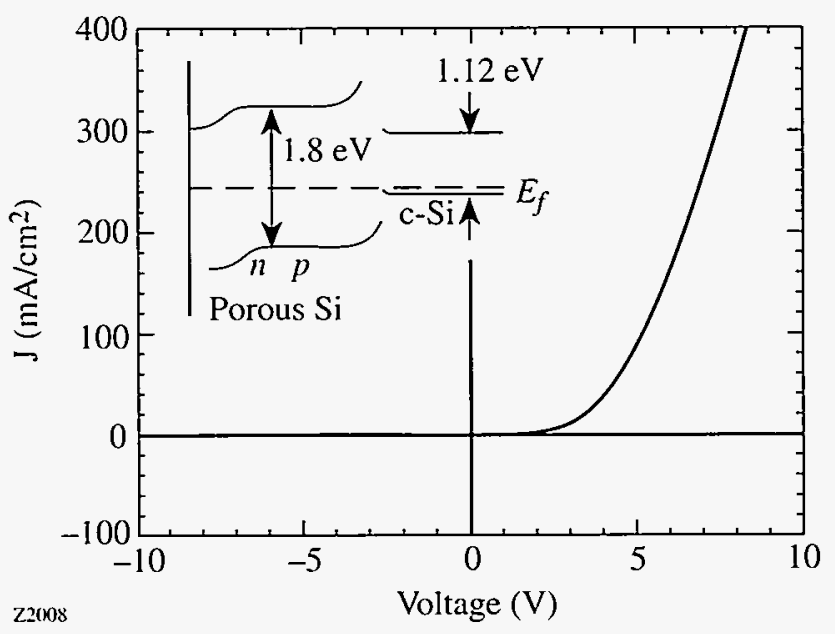

Figure 62.45

Current-voltage relationship of a light-emitting porous silicon $p$ - $n$ junction LED. The inset shows the proposed band diagram. the LEPSi layer, the backside of the wafers was heavily doped with boron, and a sintered $\mathrm{Al}$ film was used to create an intimate backside ohmic contact. Following LEPSi layer formation, a semitransparent, $100-\AA$ gold film was deposited to form patterned $0.2-\mathrm{cm}^{2}$ contacts. Using similar structures, electroluminescence has been achieved from the near infrared to the blue with nearly constant efficiencies (e.g., $0.005 \%$ in the blue using indium contacts ${ }^{72}$ ).

The band diagram proposed in Fig. 62.45 is based on an analysis of the I-V curve, its temperature dependence, and the $\mathrm{C}$-V data. ${ }^{71}$ The Fermi level for $n$ - and $p$-type LEPSi is taken to be $\sim 0.2 \mathrm{eV}$ above and below the midgap respectively. Thus, a small amount of band-bending is created within the LEPSi layer. The presence of a $p-n$ junction in the LEPSi layer affects carrier transport, resulting in a rectifying $I-V$ relationship that is nearly exponential in forward bias [i.e., $\left.I=I_{0}\left(e^{\mathrm{eV} / n k T}-1\right)\right]$. The extracted ideality factor $n$ was found to be 2.1 at applied forward bias voltages of less than $0.5 \mathrm{~V}$ and at room temperature. At higher voltages there is a large deviation from this behavior. ${ }^{71}$

Frequency modulation measurements were performed on LEPSi LED's. ${ }^{66}$ In this experiment, a peak-to-peak ac signal of $10 \mathrm{~V}$ was applied to the device under a forward dc bias of $30 \mathrm{~V}$. The modulated EL signal, detected and amplified with a lock-in amplifier, is shown in Fig. 62.46. The output power of an $L E D$ is related to the modulation frequency $\omega$ by

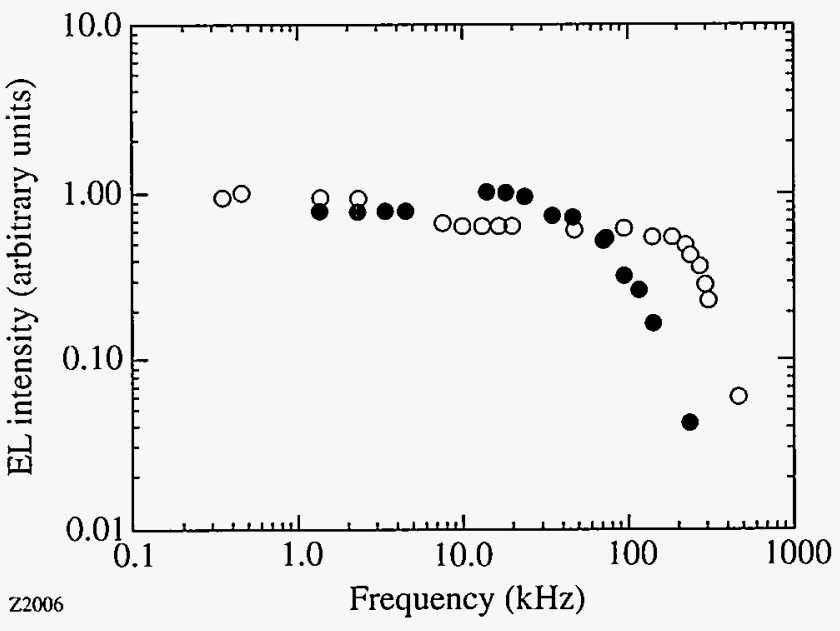

Figure 62.46

Frequency modulation response of the electroluminescence of porous silicon $p-n$ junction LED's with (O) $0.2-\mu \mathrm{m}$ junction depth and 5- $\mu \mathrm{m}$ LEPSi layer; (e) $1-\mu \mathrm{m}$ junction depth and 10- $\mu \mathrm{m}$ LEPSi layer. The 3-dB bandwidth can exceed $100 \mathrm{kHz}$. 


$$
\frac{P(\omega)}{P(0)}=\left[\frac{1}{1+\left(\omega / \omega_{0}\right)^{2}}\right]^{1 / 2}
$$

where $P(0)$ is the dc power, $P(\omega)$ is the power at frequency $\omega$, and $\omega_{0}$ is the cutoff frequency. Table 62 .V shows the relationship between the cutoff frequency of different device structures and the corresponding PL lifetime $\tau_{\mathrm{PL}}$. The fact that $2 \pi / \omega_{0}$ is comparable to $\tau_{\mathrm{PL}}$ suggests that the EL response is controlled by the carrier lifetime; thus, it appears that the maximum speed of the red $p-n$ junction LEPSi LED's is $\sim 1 \mathrm{MHz}$.

Table 62.V: Comparison of the EL time constant and the PL lifetime

\begin{tabular}{|c|c|c|}
\hline \hline Sample Description & $2 \pi \omega_{0}(\mu \mathrm{s})$ & $\tau_{\mathrm{PL}}(\mu \mathrm{s})$ \\
\hline Au/LEPSi, 30-min etching & 30 & 13 \\
\hline$p-n, 1 \mu \mathrm{m}, 10-$ min etching & 15 & 17 \\
\hline$p-n, 0.2 \mu \mathrm{m}, 5$-min etching & 0.86 & 0.7 \\
\hline \hline
\end{tabular}

When we prepare LEPSi MIS structures, not only is the efficiency lower but the I-V curve indicates that the transport mechanism is different and the maximum modulation speed is lower. Figure 62.47 shows that the current-voltage curve in forward bias follows a power law $I \sim V^{n}$, with $n>2$ in devices where EL is detected. ${ }^{67,73}$ Such a behavior is typical of a spacecharge-limited current. ${ }^{74}$ If the LEPSi layer can be modeled as a perfect insulator, then

$$
J=\varepsilon \varepsilon_{0} \mu \mathrm{V}^{2} / d^{3},
$$

where $\varepsilon$ is the dielectric function of LEPSi, $\varepsilon_{0}=8.85410^{-12}$ $\mathrm{F} / \mathrm{m}, \mu$ is the drift mobility in LEPSi, and $d$ is the thickness of the LEPSi layer. If deep traps or interface traps are present, the current may increase faster than $\mathrm{V}^{2}$, and if the mobility is electric field dependent, the current is no longer proportional to $\mathrm{V}^{2}$. When frequency-dependent EL measurements are performed, we find as expected that the drift time across the LEPSi layer limits the device speed to at most $10 \mathrm{KHz} .{ }^{71}$ Using these results, we have deduced a drift mobility of $\sim 10^{-5} \mathrm{~cm}^{2} / \mathrm{V}-\mathrm{s}$ in LEPSi layers prepared from 5 - to $10-\Omega$-cm $p$-type substrates under light assistance. ${ }^{71}$ Finally, we note that when the LEPSi layers were prepared so that the surface passivation involved $\mathrm{Si}-\mathrm{O}$ bonds and no Si-H bonds, the LED's did not degrade even after $100 \mathrm{~h}$ of continuous operation. ${ }^{67,73}$

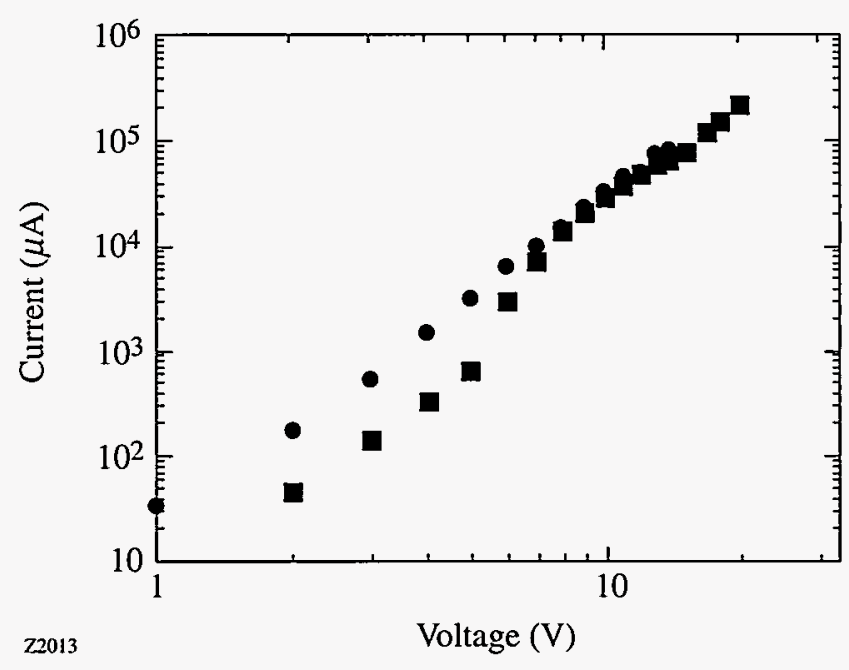

Figure 62.47

Current-voltage relationship of two metal/porous-silicon/crystalline-silicon light-emitting devices.

\section{Prospects for Commercial Devices}

Light-emitting porous silicon has given a new impetus to silicon-based optoelectronics. Since the first report of efficient photoluminescence in the red/orange part of spectrum, much progress has been achieved. Efficient luminescence has been observed from the blue/near UV to past $1.5 \mu \mathrm{m}$, as shown in Fig. 62.48. The quantum efficiency of LEPSi LED's has improved by at least four orders of magnitude since the first solid-state EL device was demonstrated. Nevertheless, much remains to be done before commercial applications of these materials becomes a reality. ${ }^{75}$ Research should focus on improving either the efficiency or the speed of LED's. Better efficiencies are required for optical display applications. In addition, to be cost competitive, displays must be made using silicon thin films, and not bulk wafers. Optical interconnect applications, in contrast, do not require extremely high efficiencies but do require data-transfer rates in excess of $1 \mathrm{Mbit} / \mathrm{s}$. Thus, for interconnects, the best hope seems to be the blue luminescence. In this section, we briefly discuss four points of relevance for technological applications. These are (1) ion implantation and oxidation of LEPSi; (2) uniformity of LEPSi; (3) mechanical stability of LEPSi; and (4) manufacture of micron-size LEPSi structures.

The major advantage of LEPSi LED's is that they could be made on the same chip as Si electronic devices or integrated circuits, which raises the important issue of their compatibility with various microelectronic processing steps. We have performed several studies ${ }^{76-80}$ that have yielded encouraging results. Ion implantation, a technique that is widely used in 


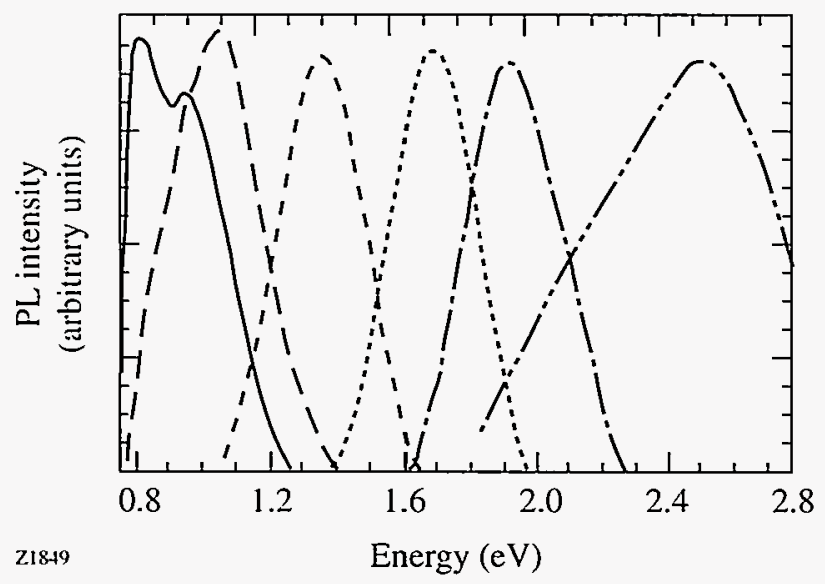

Figure 62.48

Normalized room-temperature photoluminescence spectra of various porous silicon samples. as prepared ("red" region), after oxidation ("blue" region), and after vacuum annealing ("infrared" region). The cutoff near $1.5 \mu \mathrm{m}$ is due to the $\mathrm{Ge}$ photodetector.

semiconductor technology, consists of bombarding the wafer with donor or acceptor ions to produce the desired doping profile. We have demonstrated ${ }^{76,77}$ that LEPSi can retain its strong photoluminescence after ion implantation with phosphorus and boron, provided that the dose does not exceed $-10^{14} \mathrm{~cm}^{-2}$. Another important processing step is oxidation. As was discussed previously, oxidation at elevated temperatures $\left(T_{\mathrm{ox}} \geq 1000^{\circ} \mathrm{C}\right)$ produces LEPSi that emits in the blue. If the oxidation is performed at very low temperatures $\left(T_{\mathrm{ox}} \leq\right.$ $600^{\circ} \mathrm{C}$ ), the red luminescence disappears. ${ }^{22,81}$ At these temperatures. all the hydrogen that passivated the surface of the as-grown LEPSi has been desorbed, and the oxide that is formed is of extremely poor quality. The surface is thus covered by dangling bonds, ${ }^{82}$ which act preferentially as nonradiative recombination centers. However, as $T_{\mathrm{ox}}$ is raised from $600^{\circ} \mathrm{C}$ to $\sim 1000^{\circ} \mathrm{C}$, the quality of the thermal oxide improves, the density of dangling bonds decreases, and the PL intensity recovers. Near $1000^{\circ} \mathrm{C}$, the PL intensity of highefficiency LEPSi samples recovers to within a factor of 3 of the initial intensity. The peak PL wavelength is also blue shifted after oxidation above $600^{\circ} \mathrm{C}$, in agreement with the expected decrease of the silicon crystallite size after oxidation of several silicon monolayers. ${ }^{22}$

For device applications, it is highly desirable that the porous layers have good cross-sectional and lateral uniformity. Previously published ${ }^{83.84}$ studies of depth uniformity lead us to conclude that most samples are not uniform as a function of depth, with the possible exception of thin LEPSi layers prepared in the dark using $p$-type substrates. Spatially resolved photoluminescence and Raman measurements have shown that the first few microns usually possess a different nanostructure from that of the deeper layers. As a result, both the PL spectrum and its intensity change as a function of depth. However, we have shown $n^{50,51}$ that manufacturing of LEPSi layers thicker than $10 \mu \mathrm{m}$ with homogeneous cross-sectional PL properties is possible under specific conditions. More work must be done in this area to identify the conditions that lead to uniform LEPSi layers with good luminescence efficiency. Another issue is lateral uniformity, where we distinguish three length scales. Uniformity over large areas $\left(\gg>1 \mathrm{~cm}^{2}\right)$ can be maintained if the electrochemical cell is designed carefully. On a scale of 1 to $100 \mu \mathrm{m}$, however, uniformity is more difficult to maintain. High-porosity layers tend to crack when they are removed from the solution and dried, which leads to a surface with a morphology reminiscent of a dry lake bed. ${ }^{83}$ Spatially resolved photoluminescence (SRPL) maps of such surfaces taken with $1-\mu \mathrm{m}$ spatial resolution show large differences in the local PL intensity, with regions that appear dark (no PL) and bright (visible PL). Even layers that show no evidence of cracking are often not homogeneous on a micron scale. Using SRPL maps, we have shown ${ }^{83}$ that the surface of apparently uniform samples is in fact made of a very large number of small $(\sim 1-\mu \mathrm{m})$ regions that emit very bright PL and are separated by $\geq 1 \mu \mathrm{m}$. With the knowledge that longer anodization times generally produce less homogeneous surfaces, we have been able to produce LEPSi layers that appear homogeneous on a $1-\mu \mathrm{m}$ scale by properly choosing the anodization parameters. ${ }^{83}$ Perfect uniformity on a scale of 10 to $100 \mathrm{~nm}$ is almost impossible to achieve since the crystallite sizes are in the nanometer range, although the results of Fig. 62.42 show that, at least under specific conditions, the surface can be of optical quality.

As mentioned previously, high-porosity samples have a tendency to crack when dried. In addition, all LEPSi samples, with the exception of those that have been oxidized at elevated temperatures, have poor mechanical properties. A method that improves the mechanical properties and allows the production of high-porosity $(\geq 95 \%$ ) films without cracking and degradation of the crystallinity has been demonstrated. ${ }^{85}$ In this technique, the as-prepared layers are supercritically dried in $\mathrm{CO}_{2}$ for a period of several hours. This "gentle" drying, which maintains the structural integrity of highly porous materials, has been previously used in sol-gel technology. Supercritical drying of porous silicon appears promising. Further work will help determine whether it is a necessary step for the development of LEPSi LED's. 
Finally, if LEPSi LED's are to be integrated with silicon microelectronic circuits for optical interconnects or other applications, at least two developments are necessary:

- the size of the LED's must decrease from $\sim 1 \mathrm{~mm}^{2}$ to a few $\mu \mathrm{m}^{2}$; and

- the regions of the wafer where porous silicon is not produced should be protected during anodization.

Very few studies have addressed these important issues. ${ }^{78,80,86}$ We have developed several processes that allow us to make LEPSi lines narrower than $0.5 \mu \mathrm{m}$ and to protect the adjacent silicon material (see Fig. 62.49). Although we have yet to demonstrate a practical device employing this feature, the ability to produce such ultranarrow lines suggests that integration of miniature porous silicon LED's with conventional VLSI is indeed possible.

(a)

(1)

(3)

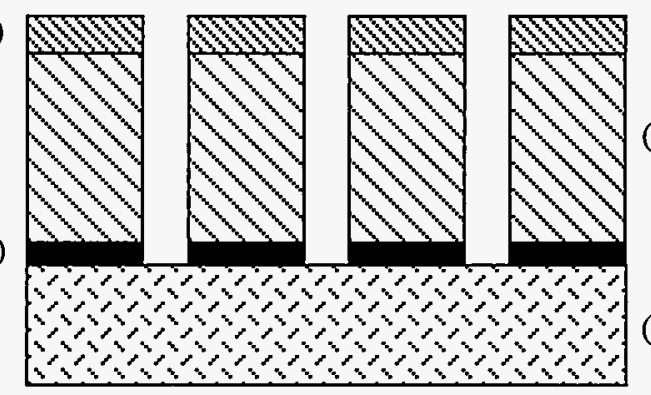

(b)

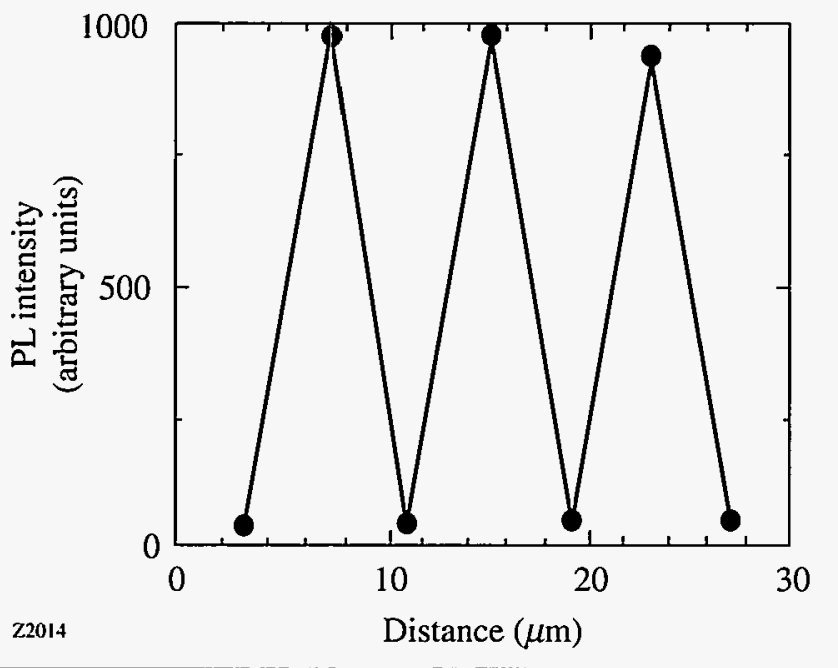

Figure 62.49

(a) Pre-anodization schematic of the mask structure. Layer (4) c-Si substrate,

(3) 100-nm-thick $\mathrm{Si}_{3} \mathrm{~N}_{4}$, (2) 5- $\mu$ m-thick crosslinked photoresist, and (1) $1-\mu$ m-thick capping resist. (b) PL map after anodization.

\section{Conclusions}

Porous silicon, a promising new material for silicon optoelectronics, has been demonstrated to photoluminesce at room temperature with an efficiency between $0.1 \%$ and $10 \%$. Its luminescence spectrum, intensity, and lifetime are highly sensitive to the growth and processing parameters. Lightemitting devices (LED's) made of porous silicon have already been demonstrated throughout the visible range. Since the peak of the photoluminescence spectrum of porous silicon can be changed from the blue/violet to wavelengths past $1.5 \mu \mathrm{m}$, it is conceivable that LED's will be made over the same range of wavelengths. Despite some impressive progress, much work still remains to be done before commercial LED's can be envisioned, even in the visible range. Nevertheless, because of its compatibility with silicon microelectronics, porous silicon is a material worthy of further scientific and technological investigations.

\section{ACKNOWLEDGMENTS}

This work was supported by grants from Rochester Gas \& Electric and the New York State Energy Research \& Development Authority, with additional support from the National Science Foundation Center for Photo-Induced Charge Transfer, Xerox, and Link fellowships.

\section{REFERENCES}

1. S. S. Iyer and Y.-H. Xie, Science 260, 40 (1993).

2. J. C. Sturm et al., in Silicon-Based Optoelectronic Materials, edited by M. A. Tischler et al., Materials Research Society Symposium Proceedings, Vol. 298 (Materials Research Society, Pittsburgh, PA, 1993), pp. 69-78.

3. P. L. Bradfield, T. G. Brown, and D. G. Hall, Appl. Phys. Lett. 55, 100 (1989).

4. F. Y. G. Ren et al., in Silicon-Based Optoelectronic Materials, edited by M. A. Tischler et al., Materials Research Society Symposium Proceedings, Vol. 298 (Materials Research Society, Pittsburgh, PA, 1993), pp. 415-423.

5. A. Uhlir, Jr., Bell Syst. Tech. J. 35, 333 (1956).

6. G. Bomchil, A. Halimasui, and R. Herino, Microelectron. Eng. 8, 293 (1988).

7. C. Pickering et al., J. Phys. C: Solid State Phys. 17, 6535 (1984).

8. L. T. Canham, Appl. Phys. Lett. 57, 1046 (1990).

9. V. Lehmann and U. Gosele, Appl. Phys. Lett. 58, 856 (1991).

10. R. L. Smith and S. D. Collins, J. Appl. Phys. 71, R1 (1992).

11. H. Koyama and N. Koshida, J. Appl. Phys. 74, 6365 (1993). 
12. Light Emission from Silicon, edited by S. S. Iyer, R. T. Collins, and L. T. Canham. Materials Research Society Symposium Proceedings, Vol. 256 (Materials Research Society, Pittsburgh, PA, 1992).

13. Microcrystalline Semiconductors: Materials Science \& Devices, edited by P. M. Fauchet et al., Materials Research Society Symposium Proceedings. Vol. 283 (Materials Research Society, Pittsburgh, PA. 1993).

14. Silicon-Based Optoelectronic Materials, edited by M. A. Tischler et al. . Materials Research Society Symposium Proceedings, Vol. 298 (Materials Research Society, Pittsburgh, PA, 1993).

15. Optical Properties of Low Dimensional Silicon Structures, edited by D. C. Bensahel. L. T. Canham, and S. Ossicini, NATO ASI Series E. Applied Sciences. Vol. 244 (Kluwer Academic Publishers, Dordrecht, 1993).

16. Light Emission from Silicon, edited by J.-C. Vial, L. T. Canham, and W. Lang. (North-Holland, Amsterdam, 1994).

17. A. G. Cullis and L. T. Canham, Nature 353, 335 (1991).

18. J. C. Vial et al., in Microcrystalline Semiconductors: Materials Science \& Devices, edited by P. M. Fauchet et al., Materials Research Society Symposium Proceedings. Vol. 283 (Materials Research Society, Pittsburgh. PA. 1993), pp. 241-246.

19. Y. Kanemitsu. Phys. Rev. B 48, 12357 (1993).

20. P. M. Fauchet, C. Peng, L. Tsybeskov, J. Vandyshev, A. Dubois, A Raisanen. T. E. Orlowski, L. J. Brillson, J. E. Fouquet, S. L. Dexheimer, J. M. Rehm. G. L. McLendon, E. Ettedgui, Y. Gao, F. Seiferth, and S. K. Kurinec, in Semiconductor Silicon/94: Proceedings of the 7th International Symposium on Silicon Materials Science and Technology, edited by H. R. Huff, W. Bergholz, and K. Sumino (The Electrochemical Society. Pennington, 1994), pp. 499-510.

21. L. Tsybeskov, C. Peng, S. P. Duttagupta, E. Ettedgui, Y. Gao, P. M. Fauchet. and G. E. Carver, in Silicon-Based Optoelectronic Materials, edited by M. A. Tischler et al., Materials Research Society Symposium Proceedings. Vol. 298 (Materials Research Society, Pittsburgh, PA, 1993). pp. 307-311.

22. C. Peng. L. Tsybeskov, and P. M. Fauchet, in Microcrystalline Semiconductors: Materials Science \& Devices, edited by P. M. Fauchet et al.. Materials Research Society Symposium Proceedings, Vol. 283 (Materials Research Society, Pittsburgh, PA, 1993), pp. 121-126.

23. L. Tsybeskov and P. M. Fauchet. Appl. Phys. Lett. 64, 1983 (1994).

24. F. Koch et al., in Microcrystalline Semiconductors: Materials Science \& Devices, edited by P. M. Fauchet et al., Materials Research Society Symposium Proceedings, Vol. 283 (Materials Research Society, Pittsburgh, PA, 1993). pp. 197-202; F. Koch, in Silicon-Based Optoelectronic Materials, edited by M. A. Tischler et al., Materials Research Society Symposium Proceedings, Vol. 298 (Materials Research Society, Pittsburgh. PA. 1993), pp. 319-329.

25. Microcrystalline and Nanocrystalline Semiconductors, edited by R. W. Collins et al.. Materials Research Society Symposium Proceedings, Vol. 358 (Materials Research Society, Pittsburgh, PA, 1995).

26. P. D. J. Calcott et al., J. Lumin. 57, 257 (1993).
27. P. D. J. Calcott et al., in Microcrystalline and Nanocrystalline Semiconductors, edited by R. W. Collins et al., Materials Research Society Symposium Proceedings, Vol. 358 (Materials Research Society, Pittsburgh, PA, 1995), pp. 465-476.

28. T. van Buuren et al., in Microcrystalline and Nanocrystalline Semiconductors, edited by R. W. Collins et al., Materials Research Society Symposium Proceedings, Vol. 358 (Materials Research Society, Pittsburgh, PA, 1995), pp. 44l-446.

29. S. Schuppler et al., Phys. Rev. Lett. 72, 2648 (1994).

30. M. B. Robinson et al., Appl. Phys. Lett. 61, 1414 (1992).

31. R. T. Collins, M. A. Tischler, and J. H. Stathis, Appl. Phys. Lett. 61, 1649 (1992).

32. V. Petrova-Koch et al., in Microcrystalline Semiconductors: Materials Science \& Devices, edited by P. M. Fauchet et al., Materials Research Society Symposium Proceedings, Vol. 283 (Materials Research Society, Pittsburgh, PA, 1993), pp. 179-184.

33. Y. Kanemitsu et al., in Silicon-Based Optoelectronic Materials, edited by M. A. Tischler et al., Materials Research Society Symposium Proceedings, Vol. 298 (Materials Research Society, Pittsburgh, PA, 1993), pp. 205-210.

34. L. Tsybeskov, J. V. Vandyshev, and P. M. Fauchet, Phys. Rev. B 49, 7821 (1994).

35. J. H. Stathis and M. A. Kastner, Phys. Rev. B 35, 2972 (1987).

36. A. Anedda et al., J. Appl. Phys. 74, 6993 (1993).

37. J. M. Rehm, G. L. McLendon, L. Tsybeskov, and P. M. Fauchet, to appear in Applied Physics Letters.

38. H. Tamura et al., Appl. Phys. Lett. 65, 1537 (1994).

39. R. E. Hummel, P. M. Fauchet, M. H. Ludwig, Ju. V. Vandyshev, S.-S. Chang, and L. Tsybeskov, to appear in Solid State Communications.

40. C. H. Perry et al., in Light Emission from Silicon, edited by S. S. Iyer, R. T. Collins, and L. T. Canham, Materials Research Society Symposium Proceedings, Vol. 256 (Materials Research Society, Pittsburgh, PA, 1992), pp. 153-158.

41. P. M. Fauchet, E. Ettedgui, A. Raisanen, L. J. Brillson, F. Seiferth, S. K. Kurinec, Y. Gao, C. Peng, and L. Tsybeskov, in Silicon-Based Optoelectronic Materials, edited by M. A. Tischler et al., Materials Research Society Symposium Proceedings, Vol. 298 (Materials Research Society, Pittsburgh, PA, 1993), pp. 271-276.

42. G. Mauckner et al., in Microcrystalline and Nanocrystalline Semiconductors, edited by R. W. Collins et al., Materials Research Society Symposium Proceedings, Vol. 358 (Materials Research Society, Pittsburgh, PA, 1995), pp. 489-494.

43. P. M. Fauchet, C. Peng, L. Tsybeskov, Ju. V. Vandyshev, A. Dubois, L. McLoud, S. P. Duttagupta, J. M. Rehm, G. L. McLendon, E. Ettedgui, Y. Gao, F. Seiferth, S. K. Kurinec, A. Raisanen, T. E. Orlowski, L. J. Brillson, and G. E. Carver, in Advanced Photonics Materials for Information Technology, edited by S. Etemad (SPIE, Bellingham, WA, 1994), Vol. 2144, pp. 34-50. 
44. M. Lannoo, presented at the International School on Luminescence of Porous Silicon and Silicon Nanostructures, Les Houches, France, February 1994; M. Lannoo, C. Delerue, and G. Allan, J. Lumin. 57, 243 (1993).

45. T. Gong, W. L. Nighan, Jr., and P. M. Fauchet, Appl. Phys. Lett. 57, $2713(1990)$.

46. J. F. Young, T. Gong, P. M. Fauchet, and P. J. Kelly, Phys. Rev. B 50, 2208 (1994).

47. P. M. Fauchet, A. Antonetti, D. Hulin, J. Kolodzey, A. Migus, and S. Wagner, Phys. Rev. Lett. 57, 2438 (1986).

48. P. M. Fauchet, D. Hulin, R. Vanderhaghen, A. Mourchid, and W. L. Nighan, Jr., J. Non-Cryst. Solids 141, 76 (1992).

49. P. M. Fauchet, Y. Kostoulas, Ju. V. Vandyshev, and V. Petrova-Koch, in Ultrafast Phenomena IX, edited by P. F. Barbara et al. (SpringerVerlag, Berlin, 1994), p. 283.

50. J. von Behren, K. B. Ucer, L. Tsybeskov, Ju. V. Vandyshev, and P. M. Fauchet, to appear in the Journal of Vacuum Science and Technology (1995).

51. J. von Behren, L. Tsybeskov, and P. M. Fauchet, Appl. Phys. Lett. 66, 1662 (1995)

52. J. L. Oudar et al., Phys. Rev. Lett. 55, 2074 (1985).

53. M. C. Downer and C. V. Shank, Phys. Rev. Lett. 56, 761 (1986).

54. P. M. Fauchet and W. L. Nighan, Jr., Appl. Phys. Lett. 48, 721 (1986)

55. A. Mourchid, D. Hulin, R. Vanderhaghen, W. L. Nighan, Jr., K. Gzara, and P. M. Fauchet, Solid State Commun. 74, 1197 (1990).

56. M. S. Hybertsen, Phys. Rev. Lett. 72, 1514 (1994).

57. A. Richter et al., IEEE Electron Device Lett. 12, 691 (1991).

58. N. M. Kalkhoran, F. Namavar, and H. P. Maruska, in Light Emission from Silicon, edited by S. S. Iyer, R. T. Collins, and L. T. Canham, Materials Research Society Symposium Proceedings, Vol. 256 (Materials Research Society, Pittsburgh, PA, 1992), pp. 89-94.

59. E. Bassous et al., in Light Emission from Silicon, edited by S. S. Iyer, R. T. Collins, and L. T. Canham, Materials Research Society Symposium Proceedings, Vol. 256 (Materials Research Society, Pittsburgh, PA, 1992), pp. 23-26.

60. N. Koshida and H. Koyama, in Light Emission from Silicon, edited by S. S. Iyer, R. T. Collins, and L. T. Canham, Materials Research Society Symposium Proceedings, Vol. 256 (Materials Research Society, Pittsburgh, PA, 1992), pp. 219-222.

61. A. Halimaoui et al., Appl. Phys. Lett. 59, 304 (1991).

62. A. Bsiesy et al., Phys. Rev. Lett. 71, 637 (1993).

63. A. G. Cullis et al., in Microcrystalline Semiconductors: Materials Science \& Devices, edited by P. M. Fauchet et al., Materials Research Society Symposium Proceedings, Vol. 283 (Materials Research Society, Pittsburgh, PA, 1993), pp. 257-262.
64. S. M. Pillai et al., Jpn. J. Appl. 2, Lett. 31, L1702 (1992).

65. L. Tsybeskov et al., unpublished data.

66. C. Peng, P. M. Fauchet, K. D. Hirschman, and S. K. Kurinec, in Microcrystalline and Nanocrystalline Semiconductors, edited by R. W. Collins et al., Materials Research Society Symposium Proceedings, Vol. 358 (Materials Research Society, Pittsburgh, PA, 1995), pp. 689-694.

67. L. Tsybeskov, S. P. Duttagupta, and P. M. Fauchet, to appear in Solid State Communications.

68. N. Koshida, presented at the International School on Luminescence of Porous Silicon and Silicon Nanostructures, Les Houches, France, February 1994.

69. P. Steiner et al., in Microcrystalline Semiconductors: Materials Science \& Devices, edited by P. M. Fauchet et al., Materials Research Society Symposium Proceedings, Vol. 283 (Materials Research Society, Pittsburgh, PA, 1993), pp. 343-351; W. Lang, P. Steiner, and F. Kozlowski, J. Lumin. 57, 341 (1993).

70. N. Koshida et al., Appl. Phys. Lett. 63, 2655 (1993).

71. C. Peng et al., submitted for publication to Applied Physics Letters.

72. W. Lang, presented at the International School on Luminescence of Porous Silicon and Silicon Nanostructures, Les Houches, France, February 1994.

73. L. Tsybeskov, S. P. Duttagupta, and P. M. Fauchet, in Microcrystalline and Nanocrystalline Semiconductors, edited by R. W. Collins et al., Materials Research Society Symposium Proceedings, Vol. 358 (Materials Research Society, Pittsburgh, PA, 1995), pp. 683-688.

74. M. A. Lampert and P. Mark, Current Injection in Solids (Academic Press, New York, 1970), p. 351.

75. P. M. Fauchet, in Porous Silicon, edited by Z. C. Feng and R. Tsu (World Scientific, Singapore, 1994), pp. 429-465.

76. C. Peng, P. M. Fauchet, J. M. Rehm, G. L. McLendon, F. Seiferth, and S. K. Kurinec, Appl. Phys. Lett. 64, 1259 (1994).

77. C. Peng, L. Tsybeskov, P. M. Fauchet, F. Seiferth, S. K. Kurinec, J. M. Rehm, and G. L. McLendon, in Silicon-Based Optoelectronic Materi$a l s$, edited by M. A. Tischler et al., Materials Research Society Symposium Proceedings, Vol. 298 (Materials Research Society, Pittsburgh, PA, 1993), pp. 179-184.

78. S. P. Duttagupta, C. Peng, P. M. Fauchet, S. K. Kurinec, and T. N. Blanton, to appear in the Journal of Vacuum Science and Technology (1995).

79. S. P. Duttagupta, L. Tsybeskov, P. M. Fauchet, E. Ettedgui, and Y. Gao, in Microcrystalline and Nanocrystalline Semiconductors, edited by R. W. Collins et al., Materials Research Society Symposium Proceedings, Vol. 358 (Materials Research Society, Pittsburgh, PA, 1995). pp. 381-386.

80. S. P. Duttagupta, P. M. Fauchet, C. Peng, S. K. Kurinec, K. Hirschman, and T. N. Blanton, in Microcrystalline and Nanocrystalline Semiconductors, edited by R. W. Collins et al., Materials Research Society 
Symposium Proceedings. Vol. 358 (Materials Research Society, Pittsburgh. PA. 1995). pp. 647-652.

81. V. Petrova-Koch et al. Appl. Phys. Lett. 61, 943 (1992).

82. H. Linke er al., in Microcrystalline Semiconductors: Materials Science \& Devices, edited by P. M. Fauchet et al., Materials Research Society Symposium Proceedings. Vol. 283 (Materials Research Society, Pittsburgh. PA. 1993). pp. 251-256.

83. E. Ettedgui. C. Peng. L. Tsybeskov, Y. Gao, P. M. Fauchet, G. E. Carver, and H. A. Mizes. in Microcrystalline Semiconductors: Materials
Science \& Devices, edited by P. M. Fauchet et al., Materials Research Society Symposium Proceedings, Vol. 283 (Materials Research Society, Pittsburgh, PA, 1993), pp. 173-178.

84. F. Kozlowski and W. Lang, J. Appl. Phys. 72, 5401 (1992).

85. L. T. Canham et al., Nature 368, 133 (1994).

86. N. M. Kalkhoran, in Microcrystalline Semiconductors: Materials Science \& Devices, edited by P. M. Fauchet et al., Materials Research Society Symposium Proceedings, Vol. 283 (Materials Research Society, Pittsburgh, PA, 1993), pp. 365-370. 


\section{Time-Dependent Reflection and Surface Temperatures during $\mathrm{CO}_{2}$ Laser Irradiation of Dental Hard Tissues with 100- $\mu$ s Pulses}

Lasers, including $\mathrm{CO}_{2}$ lasers operating around $\lambda \approx 10 \mu \mathrm{m}$, are being used frequently in dental soft tissue surgery and some dental hard tissue applications. In addition, pulsed $\mathrm{CO}_{2}$ laser irradiation has been applied successfully for in vitro caries (dental decay) prevention or retardation. ${ }^{1}$ For this application the laser is used for very localized surface heat treatment with or without inducing visible changes in the surface morphology.

For all laser applications to dental hard tissue the temperature increase in the pulpal region must be kept below $\sim 4^{\circ} \mathrm{C}$ lest the pulp be damaged. If thermally induced modifications at or near the (enamel) surface are desirable while avoiding excessive subsurface thermal assault, it is advantageous to use a laser wavelength whose absorption length is very small, i.e., whose absorption coefficient is very large. For $\mathrm{CO}_{2}$ laser radiation around $10 \mu \mathrm{m}$ the absorption coefficients are very large ${ }^{2}$ (see Fig. 62.50), ranging from $\sim 5,000 \mathrm{~cm}^{-1}$ at $10.6 \mu \mathrm{m}$ to $31,000 \mathrm{~cm}^{-1}$ at $9.6 \mu \mathrm{m}$, with corresponding absorption

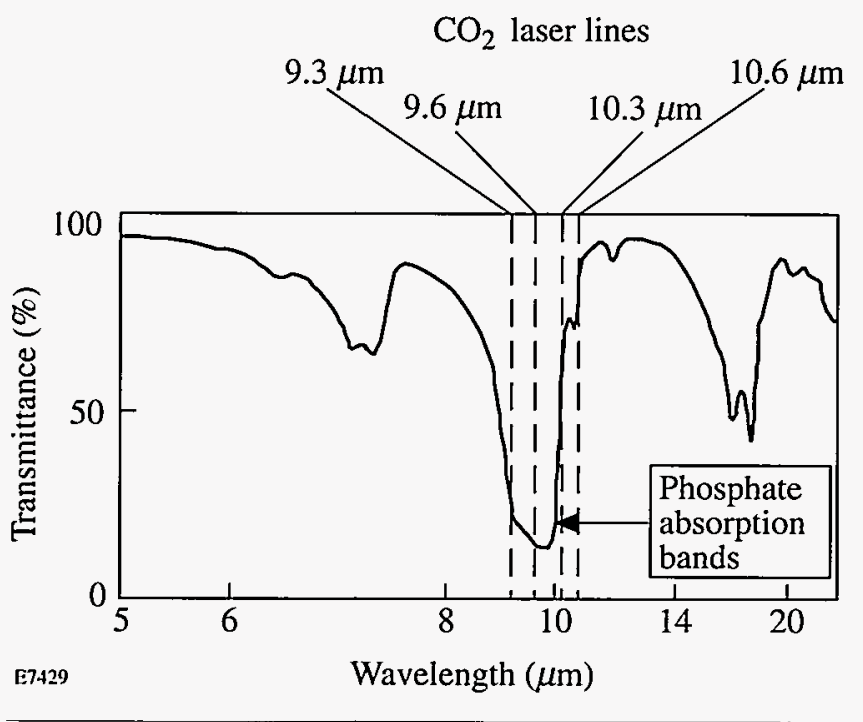

Figure 62.50

Absorption spectrum of dental enamel around the four major $\mathrm{CO}_{2}$ laser lines. The absorption band in this region is due to vibrational bands in the phosphate bonds. depths between 2 and $0.3 \mu \mathrm{m}$. These high absorption coefficients are due to intrinsic molecular vibrations of the phosphate ions ( $\mathrm{P}-\mathrm{O}$ stretching modes) of the mineral constituent (carbonated hydroxyapatite) of the dental hard tissue. Past research has found this region of the spectrum to be of particular interest for caries retardation. ${ }^{1}$

Pulses of appropriately short duration, in addition to short absorption lengths, are essential to avoid excessive subsurface heating. To avoid excessive heating at depths $\Delta x>10 \mu \mathrm{m}$, one must choose pulse durations of the order of, or less than, the relaxation time $\left(\tau \approx \Delta x^{2} / 4 \kappa \approx 60 \mu \mathrm{s}, \kappa \approx 4 \times 10^{-3} \mathrm{~cm}^{2} / \mathrm{s}=\right.$ thermal diffusivity) associated with this distance. While only approximate, these numbers are useful guides. Longer pulses or smaller absorption coefficients will heat thicker layers.

Very high absorption coefficients typically occur near resonances. All resonance transitions are typically temperature sensitive. For electronic transitions the peak absorption decreases with temperature while its width increases, but the resonance frequency remains constant. For vibrational resonances, S. S. Mitra ${ }^{3}$ shows the temperature dependence of vibrational resonances in nonmetallic solids (e.g., MgO). The peak absorption coefficient drops rapidly between room temperature and $\sim 600^{\circ} \mathrm{C}$, and the absorption band broadens and shifts toward longer wavelengths. The frequency shifts are at least partly due to the lengthening of the bond distances. The resonances are typically asymmetric, and their shapes and values depend on the particular material.

Basic optics theory relates the absorption coefficient and index of refraction through the Kramers-Kronig relations. ${ }^{4}$ The textbook index of refraction typically has a maximum on the long-wavelength side of the resonance and a minimum on its short-wavelength side. For vibrational transitions the behavior is qualitatively similar, though generally more complex. Nevertheless, as the temperature increases and the absorption decreases and shifts toward longer wavelengths, the index of refraction exhibits similar behavior, i.e., its peak values drop 
and the resonance broadens and shifts toward lower frequencies (longer wavelengths). We note that a similar temperature dependence of the strong absorption coefficient for water near $\lambda=3 \mu \mathrm{m}$ has been reported in the literature. ${ }^{5-7}$

Associated with the index of refraction, $n$, is the wellknown Fresnel reflection given by $R=(1-n)^{2} /(1+n)^{2}$. When the absorption coefficient is very strong (as it is typically near a resonance transition), the index of refraction becomes complex and can be written as $n=n_{r}+i k$, where $n_{r}$ is the real part of the index of refraction, $i=\sqrt{-1}$, and $k$ is the extinction coefficient. The extinction coefficient is related to the absorption coefficient by $\alpha=4 \pi k / \lambda$. Under these conditions the above Fresnel-reflection formula is rewritten as

$$
R=\left[\left(1-n_{r}\right)^{2}+k^{2}\right] /\left[\left(1+n_{r}\right)^{2}+k^{2}\right]
$$

We note that for either $n_{r}$ or $k$ (or both) $>>1$, the reflection coefficient approaches $100 \%$ ! In fact, for enamel at $\lambda=9.6 \mu \mathrm{m}$ we find $R \approx 50 \%$, while for $\lambda=10.6 \mu \mathrm{m}$ we find $R \approx 13 \%$. These measurements were first reported by Duplain ${ }^{2}$ for bovine enamel and have been verified in our laboratory for both bovine enamel and human enamel. Thus, if either $k$ and/or $n_{r}$ decreases due to their temperature dependence, the corresponding surface reflection decreases. While this observation certainly holds in the immediate vicinity of a resonance, farther away the reflection losses could actually increase with temperature due to the temperature dependence of the complex index of refraction. ${ }^{2}$ The most dramatic changes in both $n_{r}$. and $k$ occur at rather modest temperatures below $600^{\circ} \mathrm{C}$ to $900^{\circ} \mathrm{C}$ with changes by factors of $\geq 2$ being quite plausible. ${ }^{3}$

Since the surface temperatures during $\mathrm{CO}_{2}$ laser irradiation can easily reach $1000^{\circ} \mathrm{C}$ or more, we can expect significant changes in the reflection coefficient during the laser pulse. Concomitant with a reduced reflection is increased energy coupling to the irradiated substrate; thus, the apparently paradoxical situation can arise where the absorption coefficient decreases while the absorbed energy increases, albeit over a greater volume.

In this article we report on time-dependent reflection and surface temperature measurements for laser-irradiated dental hard tissue at two of the four major $\mathrm{CO}_{2}$ laser wavelengths (9.6 and $10.6 \mu \mathrm{m}$ ). These wavelengths were chosen for their potential usefulness in caries retardation if appropriate irradiation conditions are chosen. ${ }^{1}$

\section{Method}

Time-resolved reflection and surface temperature measurements were carried out using the experimental setup illustrated in Fig. 62.51. The light collector for the surface temperature measurements is an elliptical, rhodium-coated mirror (left side in Fig. 62.51) with an entrance hole at the top for the $\mathrm{CO}_{2}$ laser beam. All thermal radiation emitted into the upper hemisphere was collected and imaged onto the $\mathrm{HgCdZnTe}$ detector, which has a time response of $\sim 1 \mu \mathrm{s}$. The broadband filter shields the detector against scattered laser radiation and passes the thermal radiation between 5 and $9 \mu \mathrm{m}$. The signal from the detector was acquired by a digital oscilloscope (TEK2440) and recorded by a personal computer.

The time-resolved reflection measurements were made using a gold-coated integrating sphere as a light collector (right side in Fig. 62.51) with a broadband filter in front of the detector. The filter passes the laser light and adjusts the light level at the detector for optimum detection.

The recorded pyrometric signals were converted to surface temperatures using a calibration procedure described by Fried et al. ${ }^{8}$ Synthetic carbonated hydroxyapatite (CAP) heated in a furnace at a known temperature of up to $\sim 1000^{\circ} \mathrm{C}$ was employed as a calibration standard. A known area of the sample was imaged onto the detector using the same broadband filters as in the actual experiment. The results indicate that the emissivity is close to unity, i.e., the thermal emission from our samples is close to blackbody emission. However, we have no independent measurement of the absorption depths in the region between 5 and $9 \mu \mathrm{m}$. For the moment we assume that this absorption depth is small compared to the depths over which a given temperature is measured. This assumption is tenuous and must always be kept in mind when interpreting temperature measurements.

The strong temperature dependence of the total blackbody emission $\left(A \sigma T^{4}, A=\right.$ emitting area, $\sigma=$ Stefan-Boltzmann constant, and $T=$ absolute temperature) renders such a measurement insensitive to the exact value of $A$. Likewise, this strong temperature dependence strongly biases the measurement toward the peak temperature. Our actual measurements are carried out, however, in a fixed and limited wavelength interval ( 5 to $9 \mu \mathrm{m}$ ) causing the measured signal to follow a lower-power law (smaller exponent in $T$ ), which furthermore changes with temperature. While the calibration procedure takes this effect into account, the temperatures deduced from these measurements are more sensitive to the exact value of $A$ 


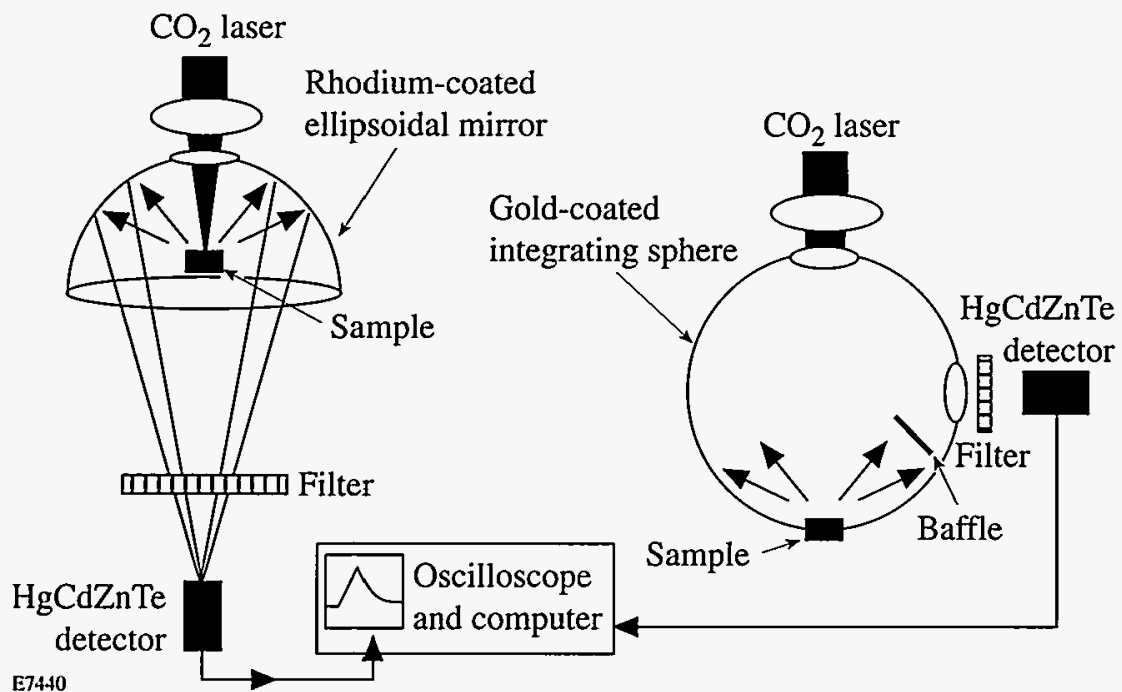

Figure 62.51

Experimental arrangement for measuring the time-dependent surface reflection (right side) and surface temperature (left side) of laserirradiated dental hard tissue. and less strongly biased to the maximum temperature in the area measured. As a consequence, assigning error bars to these measurements is rather difficult.

The tooth samples are unerupted, human third molars with a polished (1- $\mu \mathrm{m}$ grit size) enamel or dentin surface. The polished teeth are sonicated to remove the smear layer and polishing debris; the efficacy of this procedure is verified using scanning electron microscopy.

Our wavelength-tunable $\mathrm{CO}_{2}$ laser (Pulsed Systems, Los Alamos, NM) is capable of delivering up to $200 \mathrm{~mJ}$ in pulses of $50 \mu \mathrm{s}$ to $1 \mathrm{~ms}$ and is tunable from 9.3 to $9.6,10.3$, and

(a)

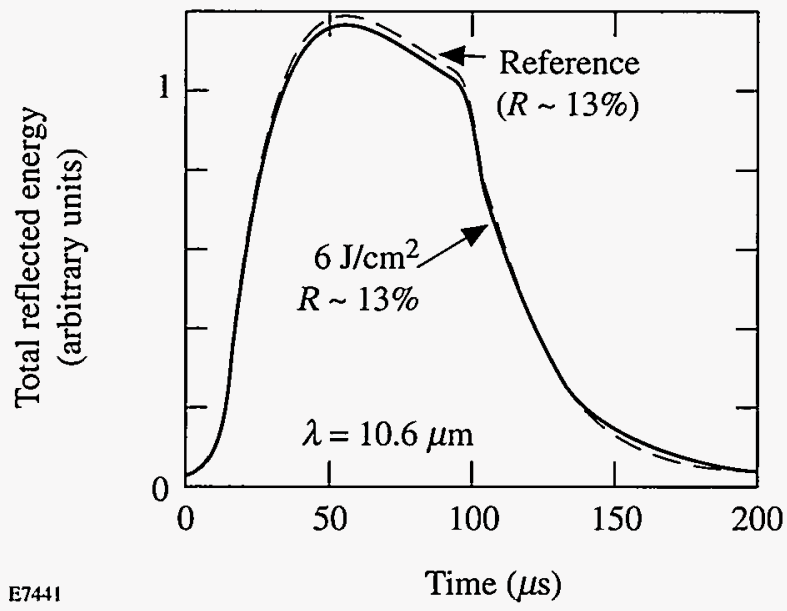

$10.6 \mu \mathrm{m}$. At present we believe that the $100-\mu$ s pulse duration is the most suitable for caries retardation applications.

\section{Results and Discussion}

1. Time-Resolved Reflection in Dental Enamel

Typical time-resolved reflection measurements of human dental enamel are shown in Fig. 62.52 for $\lambda=10.6$ and $9.6 \mu \mathrm{m}$. These wavelengths have the largest difference in absorption coefficient (see above) and therefore are expected to exhibit the largest difference in thermal behavior. For both wavelengths the reference pulse shown represents the reflection from an enamel surface irradiated at low fluence $\left(\sim 1 \mathrm{~J} / \mathrm{cm}^{2}\right)$ scaled to $6 \mathrm{~J} / \mathrm{cm}^{2}$ (i.e., six times the reflected signal at $1 \mathrm{~J} / \mathrm{cm}^{2}$ ).

(b)

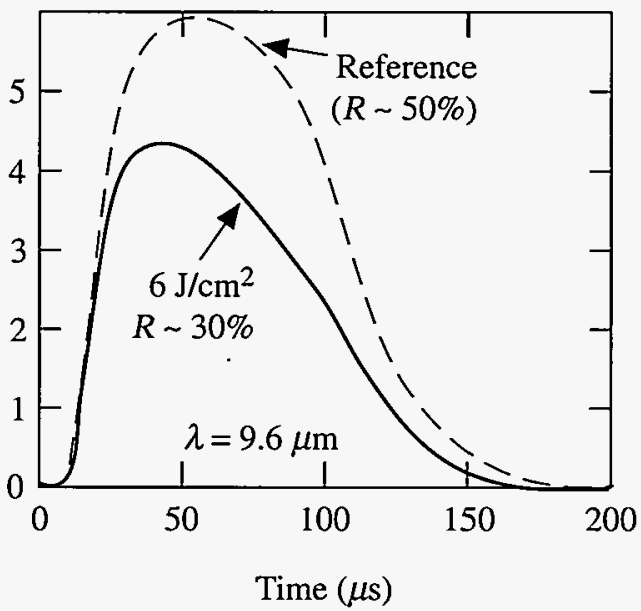

\section{Figure 62.52}

Time-resolved reflection from polished human dental enamel for two $\mathrm{CO}_{2}$ laser wavelengths at low $\left(\sim 1-\mathrm{J} / \mathrm{cm}^{2}\right)$ and high $\left(\sim 6-\mathrm{J} / \mathrm{cm}^{2}\right)$ fluence, (a) $\lambda=10.6 \mu \mathrm{m}$, (b) $\lambda=9.6 \mu \mathrm{m}$. The reference (low-fluence reflection) is linearly scaled to that expected at high fluence and mirrors the incident pulse shape. The overall lowfluence reflections are $\sim 13 \%$ and $\sim 50 \%$ at $10.6 \mu \mathrm{m}$ and $9.6 \mu \mathrm{m}$, respectively. 
This reference is identical in shape to the incident laser pulse, implying no change in reflectivity during the pulse at that fluence. For the 10.6- $\mu \mathrm{m}$ reflection data [Fig. 62.52(a)] there is essentially no change in reflectivity as the fluence is raised from $1 \mathrm{~J} / \mathrm{cm}^{2}$ to $6 \mathrm{~J} / \mathrm{cm}^{2}$. In contrast, for $\lambda=9.6 \mu \mathrm{m}$ [Fig. 62.52(b)] there is a very marked difference between the reflection signals registered for the two fluence levels. While the overall reflection loss at low fluence is $\sim 50 \%$, the reflection losses drop to $\sim 30 \%$ at $6 \mathrm{~J} / \mathrm{cm}^{2}$; thus the amount of energy actually absorbed by the enamel increases at the higher fluence. These observations are consistent with the temperature-dependent absorption and reflection coefficients that are expected to decrease for the most highly absorbing $\mathrm{CO}_{2}$ line at $\geq 6 \mathrm{~J} / \mathrm{cm}^{2}$, while remaining quite unaffected at $10.6 \mu \mathrm{m}$. At $10.6 \mu \mathrm{m}$ the absorption is low and relatively far from the main vibrational resonances (see Fig. 62.50). On the basis of qualitative similarities with $\mathrm{MgO}$ we would expect no significant changes in either the absorption or reflection coefficient this far from the main resonance. ${ }^{3}$

\section{Time-Resolved Temperatures in Dental Enamel}

Time-resolved temperature measurements show a great variety of behavior depending on whether enamel or dentin is irradiated and whether one looks at the first, second, tenth, or subsequent pulses. ${ }^{9}$ At present we believe that these initial pulse-to-pulse changes in the temperature measurements relate to burnoff (blowoff) of the organic constituents of the dental hard tissue as well as some surface melting and recrystallization of the mineral substrate. After approximately ten pulses, the surface typically stabilizes, and temporal temperatures like those shown in Fig. 62.53 are obtained reproducibly. We note that for 9.6- and 10.6- $\mu \mathrm{m}$ irradiation with single, $6-\mathrm{J} / \mathrm{cm}^{2}, 100-\mu \mathrm{s}$ pulses, the peak surface temperatures are quite similar for the two wavelengths with the peak surface temperature at $9.6 \mu \mathrm{m}$ being slightly higher. The energy coupled into the sample is smaller by $\sim 25 \%$ at $9.6 \mu$ m due to reflection losses (see preceding section). The deposited energy density near the surface is still larger, however, at $9.6 \mu \mathrm{m}$ due to the higher absorption coefficient (shorter absorption depth)even after allowing for the temperature dependence of the absorption coefficient and heat conduction into the interior of the target. The crossover of the two curves reflects the fact that at $9.6 \mu \mathrm{m}$ less energy is deposited in the sample over a shorter distance than at $10.6 \mu \mathrm{m}$. This leads to higher surface temperatures at early times, while at later times, when thermal diffusion has heated a larger part of the sample, the detailed laser-energy deposition is unimportant and the measured surface temperatures reflect only the total energy deposited. We also note that at lower fluences (such as $4 \mathrm{~J} / \mathrm{cm}^{2}$ ) the temperature profiles for

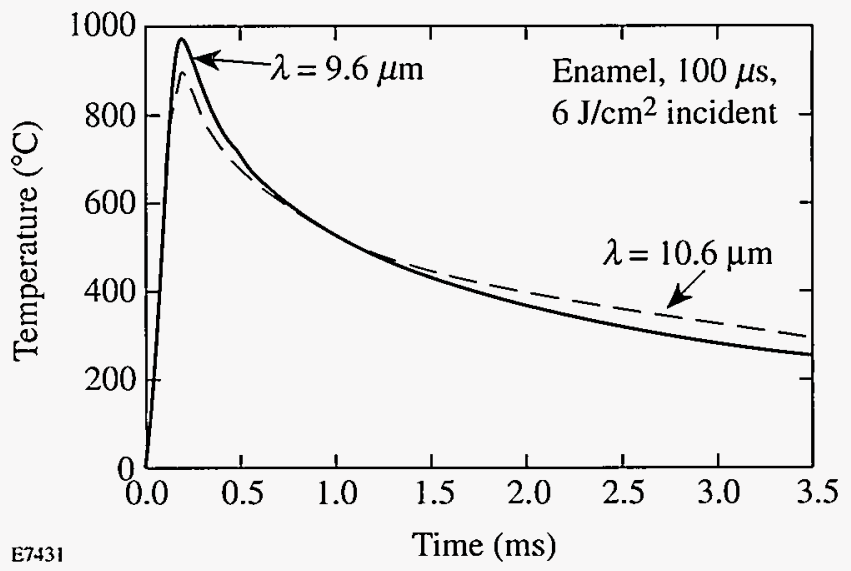

Figure 62.53

Time-resolved temperatures for enamel irradiated with $100-\mu \mathrm{s} \mathrm{CO}_{2}$ laser pulses at 9.6 and $10.6 \mu \mathrm{m}$ and a fluence of $\sim 6 \mathrm{~J} / \mathrm{cm}^{2}$. The peak surface temperature for $9.6-\mu \mathrm{m}$ irradiation is slightly larger than at $10.6 \mu \mathrm{m}$ due to the higher absorption coefficient and in spite of the higher surface-reflection losses at this wavelength.

9.6- and 10.6- $\mu \mathrm{m}$ irradiation of dental enamel are quite different, with $9.6 \mu \mathrm{m}$ giving a markedly higher peak temperature. ${ }^{9}$

\section{Time-Resolved Reflection in Dentin}

The time-resolved, high-fluence $\left(>6-\mathrm{J} / \mathrm{cm}^{2}\right)$ reflection from dentin surfaces exhibits a similar wavelength dependence to enamel. Here, too, we plot the time-resolved reflection after a number of pulses have stabilized the dentin surface. The reflection curves for prior shots are clearly affected by the blowoff of the organic constituents and water. After 20 shots, scanning electron microscope (SEM) images at $60,000 \times$ magnification reveal a melted and recrystallized mineral surface devoid of organic material and quite like that obtained with enamel. All subsequent shots cause no further material blowoff from the surface as judged either optically or acoustically. Lower-magnification SEM images show differences between multiply irradiated dentin and enamel samples with, e.g., the dentinal tubules remaining open. The differences between the reference profile and the $6-\mathrm{J} / \mathrm{cm}^{2}$ reflection profile at $9.6 \mu \mathrm{m}$ [Fig. 62.54(b)] appear noticeably smaller than for enamel [Fig. 62.52(b)]. These differences largely disappear when one takes into account that the low-fluence reflectivity of irradiated dentin increases ${ }^{9}$ from $R \approx 17 \%$ to $R \approx 22 \%$ after the dentin surface has been recrystallized by multiple irradiation at $6 \mathrm{~J} / \mathrm{cm}^{2}$. We therefore surmise that the basic absorption process is the same for irradiated enamel and dentin but the density of dentin near the surface is still somewhat lower than that of enamel. Under these conditions the overall absorption and reflection coefficients would be re- 
(a)

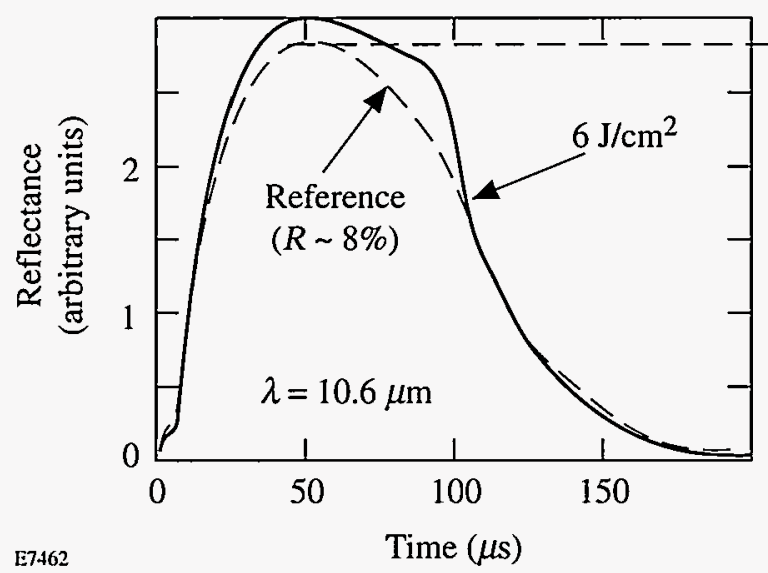

(b)

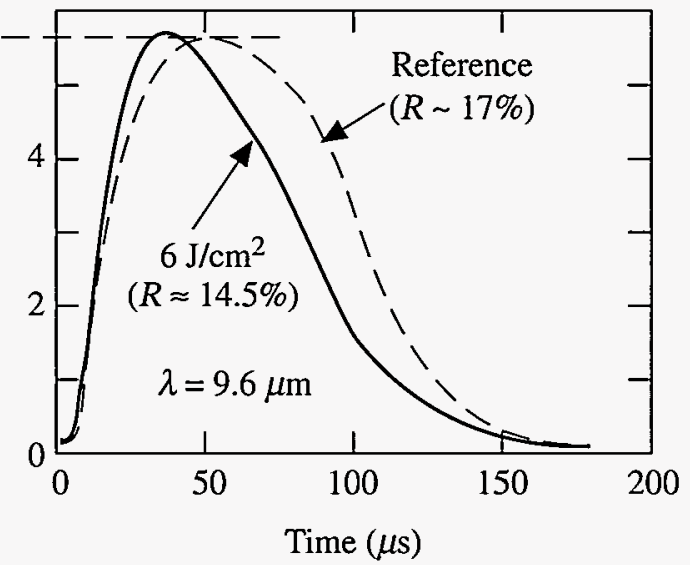

Figure 62.54

Time-resolved reflection from dentin irradiated at (a) $10.6 \mu \mathrm{m}$ and (b) $9.6 \mu \mathrm{m}$. The reference pulse is the reflection at $1 \mathrm{~J} / \mathrm{cm}^{2} \mathrm{scaled} \mathrm{to} 6 \mathrm{~J} / \mathrm{cm}^{2}$ and is identical to the incident pulse shape. The strong wavelength dependence of the reflection at $9.6 \mu \mathrm{m}$ is connected with the temperature-dependent absorption coefficient as in Fig. 62.52 .

duced, but the relative change in reflection due to the temperature-dependent absorption coefficient would remain unchanged, consistent with our observations.

\section{Conclusions}

We have observed the first experimental evidence of timedependent reflection in $\mathrm{CO}_{2}$-laser-irradiated dental hard tissue at $9.6 \mu \mathrm{m}$, while no differences in reflectivity were observed at $10.6 \mu \mathrm{m}$. Using the known relationships between surface reflection and high absorption coefficients, we have related the reduced reflection for the high-fluence, 9.6- $\mu \mathrm{m}$ data to the temperature dependence of the absorption coefficient. This temperature dependence is much more pronounced in the immediate vicinity of the strong vibrational bands of the phosphate ions $(9.6 \mu \mathrm{m})$ than farther away $(10.6 \mu \mathrm{m})$. We have also seen direct evidence of this temperature-dependent $a b-$ sorption in time-resolved temperature measurements using fast photodetectors.

These data show that an appropriate choice of wavelength and pulse duration allows fine tuning of morphological surface modifications of irradiated dental hard tissue. Such fine tuning is expected to permit optimization of the laser-irradiation conditions for the purpose of caries reduction in dental hard tissue.

\section{ACKNOWLEDGMENT}

This work was supported by NIH/NIDR Grant DE 09958.

\section{REFERENCES}

1. J. D. Featherstone and D. G. Nelson, Adv. Dent. Res. 1, 21 (1987).

2. G. Duplain, P. A. Belanger, and R. Boulay, Appl. Opt. 26, 4447 (1987).

3. S. S. Mitra, in Handbook of Optical Constants of Solids, edited by E. D. Palik (Academic Press, Orlando, 1985), Chap. 11, pp. 213-270.

4. M. Born and E. Wolf, Principles of Optics: Electromagnetic Theory of Propagation, Interference, and Diffraction of Light, 2nd rev. ed. (Macmillan, New York, 1964).

5. J. T. Walsh, Jr. and J. P. Cummings, Las. Surg. Med. 15, 295 (1994).

6. K. L. Vodop'yanov, Sov. Phys. JETP 70, 114 (1990).

7. K. L. Vodop'yanov, J. Chem Phys. 94, 5389 (1991).

8. D. Fried, S. F. Borzillary, S. M. McCormack, R. E. Glena, J. D. B. Featherstone, and W. Seka, in Laser Surgery: Advanced Characterization, Therapeutics, and Systems IV, edited by R. R. Anderson and A. Katzir (SPIE, Bellingham, WA, 1994), Vol. 2128, pp. 319-328.

9. D. Fried, R. E. Glena, J. D. B. Featherstone, and W. Seka, in Lasers in Dentistry, edited by H. A. Wigdor, J. D. B. Featherstone, and J. M. White (SPIE, Bellingham, WA, 1995), Vol. 2394, pp. 41-50. 


\title{
Publications and Conference Presentations
}

\author{
Publications
}

U. Alon, J. Hecht, D. Ofer, and D. Shvarts, "Power Laws and Similarity of Rayleigh-Taylor and Richtmyer-Meshkov Mixing Fronts at All Density Ratios," Phys. Rev. Lett. 74, 534 (1995).

R. Betti and J. P. Freidberg, "Stability Analysis of Resistive Wall Kink Modes in Rotating Plasmas," Phys. Rev. Lett. 74, 2949 (1995).

D. K. Bradley, P. M. BelI, O. L. Landen, J. D. Kilkenny, and J. Oertel, "Development and Characterization of a Pair of 30-40 ps X-Ray Framing Cameras," Rev. Sci. Instrum. 66, 716 (1995).

S.-H. Chen and S. Krishnamurthy, "Some Fundamental Issues Governing Thermotropic Chiral Nematic Copolymers," Polym. Preprints (Japan) 42, 122 (1993).

S.-H. Chen, H. Shi, and J. Mastrangelo, "Novel Low Molar Mass Glass-Forming Liquid Crystals: Synthesis, Characterization and Morphological Stability," Polym. Preprints (Japan) 43, 1674 (1994).

C. Y. Chien, G. Korn, J. S. Coe, J. Squier, G. Mourou, and R. S. Craxton, "Highly Efficient Second-Harmonic Generation of Ultra-Intense Nd:Glass Laser Pulses," Opt. Lett. 20, 353 (1995).

D. Fried, R. E. Glena, J. D. B. Featherstone, and W. Seka, "Nature of Light Scattering in Dental Enamel and Dentin at Visible and Near Infrared Wavelengths," Appl. Opt. 34, I278 (1995).

D. A. Haynes, Jr., C. F. Hooper, Jr., R. C. Mancini, D. K. Bradley, J. Delettrez, R. Epstein, and P. A. Jaanimagi, "Spectroscopic Analysis of Ar-Doped Laser-Driven Implosions," Rev. Sci. Instrum. 66, 755 (1995).
P. A. Jaanimagi, R. C. Elton, B. L. Welch, Y. Leng, and H. R. Griem, "Extending X-Ray Streak Camera Operation to Vacuum Ultraviolet Wavelengths," Rev. Sci. Instrum. 66, 713 (1995).

J. P. Knauer, R. L. Kremens, M. A. Russotto, and S. Tudman, "Using Cosmic Rays to Monitor Large Scintillator Arrays," Rev. Sci. Instrum. 66, 926 (1995).

E. M. Korenic and K. L. Marshall, "Blending Polysiloxane 'Glass Resins' to Produce Optical Films with a Specific Refractive Index," Opt. Photonics News (Supplement) 6(2) (1995).

F. J. Marshall and Q. Su, "Quantitative Measurements with $\mathrm{X}$-Ray Microscopes in Laser-Fusion Experiments," Rev. Sci. Instrum. 66, 725 (1995).

C. I. Moore, J. P. Knauer, and D. D. Meyerhofer, “Observation of the Transition from Thomson to Compton Scattering in Multiphoton Interactions with Low-Energy Electrons," Phys. Rev. Lett. 74, 2439 (1995).

J. Peatross and D. D. Meyerhofer, "Angular Distribution of High-Order Harmonics Emitted from Rare Gases at Low Density," Phys. Rev. A 51, R906 (1995).

D. Ress, L. B. DaSilva, R. A. London, J. E. Trebes, R. A. Lerche, and D. K. Bradley, "Novel X-Ray Imaging Methods at the Nova Laser Facility," Rev. Sci. Instrum. 66, 579 (1995) (invited).

L. Shi, T. Gong, W. Xiong, X. Weng, R. Sobolewski, and P. M. Fauchet, "Femtosecond Optical Spectroscopy of Partially Deoxygenated Y-Ba-Cu-O Thin Films," in Ultrafast Phenomena IX, edited by P. F. Barbara, W. H. Knox, G. A. Mourou, and A. H. Zewail, Springer Series in Chemical Physics, Vol. 60 (Springer-Verlag, Berlin, 1994), pp. 327-328. 
B. Yaakobi. R. Epstein, F. J. Marshall, D. K. Bradley, P. A. Jaanimagi, and Q. Su, "New Diagnostic Features in the Laser Implosion of Argon-Filled Targets," Rev. Sci. Instrum. 66. 728 (1995).

B. Yaakobi, D. Shvarts, F. J. Marshall, R. Epstein, and Q. Su,
"Target Imaging and Backlighting Diagnosis," Rev. Sci. Instrum. 66, 731 (1995).

B. Yaakobi, Q. Su, F. J. Marshall, and R. Epstein, "Monochromatic Backlighting as a Laser-Fusion Diagnostic," J. X-Ray Sci. Technol. 5, 73 (1995).

\section{Forthcoming Publications}

M. S. Adams, M. V. Fedorov, V. P. Krainov, and D. D. Meyerhofer, "Comparison of Quasiclassical and Exact Dipole Moments for Bound-Free Transitions in Hydrogen," to be published in Physical Review A.

T. R. Boehly, R. S. Craxton, T. H. Hinterman, J. H. Kelly, T. J. Kessler, S. A. Kumpan, S. A. Letzring, R. L. McCrory, S. F. B. Morse, W. Seka, S. Skupsky, J. M. Soures, and C. P. Verdon, "The Upgrade to the OMEGA Laser System," to be published in the Review of Scientific Instruments.

T. R. Boehly, R. S. Craxton, T. H. Hinterman, P. A. Jaanimagi, J. H. Kelly, T. J. Kessler, R. L. Kremens, S. A. Kumpan, S. A. Letzring, R. L. McCrory, S. F. B. Morse, W. Seka, S. Skupsky, J. M. Soures, and C. P. Verdon, "The Upgrade to the OMEGA Laser System," to be published in the Proceedings of the American Nuclear Society.

T. R. Boehly. R. S. Craxton, T. H. Hinterman, P. A. Jaanimagi, R. L. Keck, J. H. Kelly, T. J. Kessler, R. L. Kremens, S. A. Kumpan, S. A. Letzring, R. L. McCrory, S. F. B. Morse, W. Seka, S. Skupsky, J. M. Soures, and C. P. Verdon, "The Upgrade to the OMEGA Laser System," to be published in the Proceedings of IAEA, Paris, France, 14-18 November 1994.

X. D. Cao, L. Zheng, and D. D. Meyerhofer, "Measurement of Group-Velocity Walk-Off of Short Pulses in Nonlinear Crystals: A Novel Method," to be published in Optics Letters.

C. T. Cotton, "The Design of an All-Spherical, ThreeMirror, Off-Axis Telescope Objective," to be published in the OSA Proceedings of the International Optical Design Conference '94. Rochester, NY.

M. J. Cumbo, D. Fairhurst, S. D. Jacobs, and B. E. Puchebner, "Slurry Particle Size Evolution during the Polishing of Optical Glass," to be published in Applied Optics.
M. Currie, C.-C. Wang, D. Jacobs-Perkins, R. Sobolewski, and T. Y. Hsiang, "An Optoelectronic Testing System of Rapid, Single-Flux Quantum Circuits," to be published in IEEE Transactions on Applied Superconductivity.

W. R. Donaldson, L. Mu, D. Jacobs-Perkins, and T. Y. Hsiang, "Two-Dimensional Electro-Optic Sampling in GaAs Photoconductive Switches," to be published in the Proceedings of LEOS '94 7th Annual Meeting, Boston, MA.

J. D. B. Featherstone, N. A. Barrett-Vespone, D. Fried, Z. Kantorowitz, J. Lofthouse, and W. Seka, "Rational Choice of Laser Conditions for Inhibition of Caries Progression," to be published in the Proceedings of SPIE Biomedical Conference '95, San Jose, CA.

D. Fried, R. E. Glena, J. D. B Featherstone, and W. Seka, "Multiple Pulse Irradiation of Dental Hard Tissues at $\mathrm{CO}_{2}$ Laser Wavelengths," to be published in the Proceedings of SPIE Biomedical Conference '95, San Jose, CA.

K. Green, W. R. Donaldson, R. Sobolewski, M. D. Skeldon, W. Seka, A. Okishev, and S. A. Letzring, "Microwave Bandwidth Measurements of Illuminated Silicon Switches Used in Pulse-Shape Control for Laser-Fusion Drivers," to be published in the SPIE Proceedings of the 1st Annual International Conference on Solid-State Lasers for Application to Inertial Confinement Fusion (ICF), Monterey, CA, 30 May2 June 1995.

D. Gupta, W. R. Donaldson, and A. M. Kadin, "Transient Flux Dynamics in Optically Irradiated YBCO Thin Film Switches," to be published in IEEE Transactions on Applied Superconductivity.

D. Gupta, W. R. Donaldson, and A. M. Kadin, "Rapid Flux Motion and Critical State Dynamics in a Superconducting Disk," to be published in the Journal of Applied Physics. 
J. Hecht, D. Ofer, U. Alon, D. Shvarts, S. A. Orszag, and R. L. McCrory, "Three-Dimensional Simulations and Analysis of the Nonlinear Stage of the Rayleigh-Taylor Instability," to be published in Laser and Particle Beams.

F. A. Hegmann, D. Jacobs-Perkins, S. H. Moffat, C.-C. Wang, R. A. Hughes, M. Currie, P. M. Fauchet, T. Y. Hsiang, J. S. Preston, and R. Sobolewski, "Electro-Optic Sampling of Picosecond Photoresponse Signals from $\mathrm{YBa}_{2} \mathrm{Cu}_{3} \mathrm{O}_{7-8}$ Thin Films," to be published in Applied Physics Letters.

E. M. Korenic, S. D. Jacobs, S. M. Faris, and L. Li, "Cholesteric Liquid Crystal Inks and Paints," to be published in Research Highlights of the Army Research Office Physics Division.

Y. Kostoulas, K. B. Ucer, L. Waxer, G. W. Wicks, I. A. Walmsley, and P. M. Fauchet, "Ultrafast Carrier Lifetime in Low-Temperature-Grown GaAs, InP, and InGaP," to be published in the Proceedings of LEOS '94 7th Annual Meeting, Boston, MA, 31 October-3 November 1994.

Y. Kostoulas, L. J. Waxer, I. A. Walmsley, G. W. Wicks, and P. M. Fauchet, "Femtosecond Carrier Dynamics in LowTemperature-Grown Indium Phosphide," to be published in Applied Physics Letters.

W. Kula, W. Xiong, R. Sobolewski, and J. Talvacchio, "Laser Patterning of $\mathrm{YBa}_{2} \mathrm{Cu}_{3} \mathrm{O}_{\mathrm{x}}$ Thin Films Protected by In-Situ Grown $\mathrm{SrTiO}_{3}$ Cap Layers," to be published in IEEE Transactions on Applied Superconductivity.

W. Lang, G. Heine, W. Kula, and R. Sobolewski, "Study of Superconducting Fluctuations in $\mathrm{Bi}_{2} \mathrm{Sr}_{2} \mathrm{Ca}_{2} \mathrm{Cu}_{3} \mathrm{O}_{\mathrm{x}}$ Thin Films: Paraconductivity, Excess Hall Effect, and Magnetoconductivity," to be published in Physical Review B.

W. Lang, W. Göb, W. Kula, and R. Sobolewski, “Anisotropic Magnetoresistance in the Normal State of Oxygen-Deficient $\mathrm{YBa}_{2} \mathrm{Cu}_{3} \mathrm{O}_{7-\delta}$ Thin Films Induced by Superconducting Fluctuations," to be published in Zeitschrift Für Physik B.

Y. Lin, T. J. Kessler, and G. Lawrence, "Distributed Phase Plates for Supergaussian Focal-Plane Irradiance Profiles," to be published in Optics Letters.

R. S. Marjoribanks, F. W. Budnik, H. Chen, and D. D. Meyerhofer, "Plasma Electron Temperature in Picosecond Laser Plasmas from Quasi-Steady Ratio of Isoelectronic Lines," to be published in Physical Review Letters.
K. L. Marshall, S. D. Jacobs, and J. E. Miller, "Mid-Infrared Modulation Using Field-Induced Scattering in Ferroelectric Liquid Crystals," to be published in Applied Optics.

J. C. Mastrangelo, T. N. Blanton, and S.-H. Chen, "Crystallization upon Thermal Annealing of a Glass-Forming Liquid Crystal in the Nematic Regime," to be published in Applied Physics Letters.

R. L. McCrory, "Progress Toward Ignition with DirectDrive," to be published in Concerning Major Systems in Science and Technology.

R. L. McCrory, J. M. Soures, C. P. Verdon, T. R. Boehly, D. K. Bradley, R. S. Craxton, J. A. Delettrez, R. Epstein, P. A. Jaanimagi, S. D. Jacobs, R. L. Keck, J. H. Kelly, T. J. Kessler, H. Kim, J. P. Knauer, R. L. Kremens, S. A. Kumpan, S. A. Letzring, F. J. Marshall, P. J. McKenty, S. F. B. Morse, A. Okishev, W. Seka, R. W. Short, M. D. Skeldon, S. Skupsky, M. Tracy, and B. Yaakobi, "Direct-Drive Laser Fusion Experimental Program at the University of Rochester Laboratory for Laser Energetics," to be published in the Proceedings of the Conference on Plasma Physics and Controlled Nuclear Fusion Research.

C. J. McKinstrie, R. Betti, R. E. Giacone, T. Kolber, and E. J. Turano, "Two-Dimensional Stimulated Raman Scattering of Short Laser Pulses," to be published in Physical Review E.

D. D. Meyerhofer, J. P. Knauer, S. J. McNaught, and C. I. Moore, "Observation of Relativistic Quiver Effects during High-Intensity Laser-Electron Interactions," to be published in the Journal of the Optical Society of America B.

S. S. Papernov and A. W. Schmid, "A Comparison of LaserInduced Damage Morphology in Three Model Thin-Film Systems: $\mathrm{HfO}_{2}, \mathrm{Y}_{2} \mathrm{O}_{3}$, and $\mathrm{Ta}_{2} \mathrm{O}_{5}$," to be published in the Proceedings of the XXVI Annual Symposium on Optical Materials for High Power Lasers.

J. Peatross and D. D. Meyerhofer, "Intensity-Dependent Atomic Phase Effects in High-Order Harmonic Generation," to be published in Physical Review A.

J. Z. Roach, A. Ninkov, S. W. Swales, and T. Morris, "Design and Evaluation of a Screen CCD Imaging System," to be published in Optical Engineering. 
W. Seka, D. Fried, J. D. B. Featherstone, and S. F. Borzillary, "Light Deposition and Thermal Response in Dental Hard Tissue," to be published in the Journal of Dental Research.

W. Seka, D. Fried, J. D. B. Featherstone, and R. E. Glena, "Time-Dependent Reflection and Surface Temperatures during $\mathrm{CO}_{2}$ Laser Irradiation of Dental Hard Tissues with 50-500 $\mu$ s Pulses," to be published in the Proceedings of SPIE's Biomedical Conference '95, San Jose, CA.

H. Shi and S.-H. Chen, "Novel Glass-Forming Liquid Crystals. 2. Systems Containing High Optical Birefringence Moiety 1-(Phenyl)-2-(6-Cyanonaphth-2-yl)Ethyne," to be published in Liquid Crystals.

H. Shi and S.-H. Chen, "Effects of Stereochemistry, Mesogenic Core, and Space Length on Crystallization from Nematic and Isotropic Melts of Cyclohexane-Based, Glass-Forming Liquid Crystals," to be published in Liquid Crystals.

D. Shvarts, U. Alon, D. Ofer, R. L. McCrory, and C. P. Verdon, "Nonlinear Evolution of Multimode Rayleigh-Taylor Instability in Two and Three Dimensions," to be published in Physics of Plasmas.

A. Simon, "Parametric Excitation of Bernstein Modes in LaserProduced Plasma," to be published in Physics of Plasmas.

M. D. Skeldon, "Transverse Modulational Instabilities in the Presence of Stimulated Rotational Raman Scattering with a High-Energy Laser," to be published in Optics Letters.

D. J. Smith, J. F. Anzellotti, A. W. Schmid, S. Papernov, Z. R. Chrzan, and S. J. Van Kerkhove, "Damage Fluence at $1054 \mathrm{~nm}$ and $351 \mathrm{~nm}$ of Coatings Made with Hafnium Oxide Evaporated from Metallic Hafnium," to be published in the Proceedings of the XXVI Annual Symposium on Optical Materials for High Power Lasers, Boulder, CO.

R. Sobolewski and T. Y. Hsiang, "Progress in Ultrafast Superconducting Electronics," to be published in the Proceedings of the International Workshop on Superconductivity and Particle Detection, Toledo, $\mathrm{OH}$ (invited).

E. A. Startsev and C. J. McKinstrie, "Wave Propagation in a Drifting Plasma," to be published in Physics of Plasmas.

C. J. Twomey, T. N. Blanton, K. L. Marshall, S. H. Chen, and S. D. Jacobs, "Some Dynamic Features of the Preparation of Liquid Crystalline Elastomers," to be published in Liquid Crystals.

C. P. Verdon and R. L. McCrory, "Direct-Drive Capsule Physics," to be published in the Proceedings of ECLIM '94.

C.-C. Wang, M. Currie, and T. Y. Hsiang, "Ultrafast, Integrable, Optics-Based Interface between Superconducting and Room-Temperature Electronics," to be published in IEEE Transactions on Applied Superconductivity.

C.-C. Wang, M. Currie, R. Sobolewski, and T. Y. Hsiang, "Subpicosecond Electrical Pulse Generation by Edge Illumination of Silicon and Indium Phosphide Photoconductive Switches," to be published in Applied Physics Letters.

C.-C. Wang, M. Currie, D. Jacobs-Perkins, M. J. Feldman, R. Sobolewski, and T. Y. Hsiang, "Optoelectronic Generation and Detection of Single-Flux-Quantum Pulses," to be published in Applied Physics Letters.

M. D. Wittman, R. Q. Gram, H. Kim, C. K. Immesoete, S. G. Noyes, and S. Scarantino, "Increased Retention Time for Hydrogen and Other Gases by Polymer Shells Using Optically Transparent Aluminum Layers," to be published in the Journal of Vacuum Science and Technology.

W. Xiong, Y. Kostoulas, X. Weng, P. M. Fauchet, and R. Sobolewski, "Femtosecond Study of Electronic Structure in Semiconducting Y-Ba-Cu-O," to be published in Physical Review B.

B. Yaakobi, D. Shvarts, R. Epstein, and Q. Qu, "X-Ray Backlighting Imaging of Mixed Imploded Targets," to be published in the Journal of Applied Physics.

M. Yu, C. J. McKinstrie, and G. P. Agrawal, "Modulational Instabilities in Dispersion-Flattened Fibers," to be published in Physical Review E.

M. Yu, G. P. Agrawal, and C. J. McKinstrie, "Pump-Wave Effects on the Propagation of Noisy Signals in Nonlinear Dispersive Media," to be published in the Journal of the Optical Society of America B.

L. Zheng and D. D. Meyerhofer, "A Linear Cross-Correlation Technique for Single-Shot Measurements of Weak Light Pulses," to be published in Optics Letters. 
X. Zhou, S. Alexandrou, and T. Y. Hsiang, "Monte Carlo Investigation of the Mechanism of Subpicosecond Pulse Generation by Nonuniform Gap Illumination," to be published in Applied Physics Letters.
J. D. Zuegel and W. Seka, "Direct Measurements of LowerLevel Lifetime in Nd:YLF," to be published in the Bulletin of the American Physical Society and the IEEE Journal of Quantum Electronics.

\section{Conference Presentations}

A. Okishev, M. D. Skeldon, S. A. Letzring, W. Seka, and I. Will, "Nd:YLF Regenerative Amplifier for Pulse Shaping System of the 30-kJ (40-TW) UV OMEGA Laser System," OSA Topical Meeting: Advanced Solid-State Lasers, Memphis, TN, 30 January-2 February 1995.

The following presentations were made at the SPIE Conference-Biomedical Optics Conference '95, San Jose, CA, 5-10 February 1995:

J. D. B. Featherstone, N. A. Barrett-Vespone, D. Fried, Z. Kantorowitz, J. Lofthouse, and W. Seka, "Rational Choice of Laser Conditions for Inhibition of Caries Progression."

D. Fried, R. E. Glena, J. D. B Featherstone, and W. Seka, "Multiple Pulse Irradiation of Dental Hard Tissues at $\mathrm{CO}_{2}$ Laser Wavelengths."

W. Seka, D. Fried, J. D. B. Featherstone, and R. E. Glena, "Time-Dependent Reflection and Surface Temperatures during $\mathrm{CO}_{2}$ Laser Irradiation of Dental Hard Tissues with 50-500 $\mu$ s Pulses."

The following presentations were made at the Tenth Target Fabrication Specialists' Meeting, Taos, NM, 6-10 February 1995:

C. M. Chen, H. Kim, M. Wittman, and S. Letzring, "Design of a Resonant Cavity for Plasma Heating Experiments."

H. Kim, E. L. Alfonso, and S.-H. Chen, "Fabrication of Plastic Shells by an Improved Microencapsulated Technique."

M. D. Wittman, H. Kim, and A. S. Chow, "Determination of the Wall Thickness and Uniformity of Inertial-Fusion Cap- sules Using the Self-Interference Fringes Produced with Narrow-Bandwidth Illumination."

R. L. McCrory, Jr., "Direct-Drive Scaling for High Gain," AAAS Meeting (American Association for the Advancement of Science), Atlanta, GA, 19 February 1995.

The following presentations were made at the Ultrafast Electronics and Optoelectronics Topical Meeting, Dana Point, CA, 13-15 March 1995:

F. A. Hegmann, S. H. Moffat, R. A. Hughes, J. S. Preston, D. Jacobs-Perkins, C.-C. Wang, T. Y. Hsiang, and R. Sobolewski, "Electro-Optic Sampling of Picosecond Photoresponse of Epitaxial $\mathrm{YBa}_{2} \mathrm{Cu}_{3} \mathrm{O}_{7-\mathrm{x}}$ Thin Films."

D. Jacobs-Perkins, M. Currie, C.-C. Wang, R. Sobolewski, M. J. Feldman, and T. Y. Hsiang, "First Direct Observation of Single-Flux Quantum Pulses.”

C.-C. Wang, M. Currie, and T. Y. Hsiang, "All-Silicon, Ultrafast, Integrable Optoelectronic Interface."

C.-C. Wang, M. Currie, R. Sobolewski, and T. Y. Hsiang, "Picosecond Pulse Generation by Edge Illumination of Si and InP Photoconductive Switches."

F. A. Hegmann, D. Jacobs-Perkins, S. H. Moffat, C.-C. Wang, R. A. Hughes, M. Currie, P. M. Fauchet, T. Y. Hsiang, J. S. Preston, and R. Sobolewski, "Electro-Optic Sampling of Picosecond Photoresponse Signals from $\mathrm{YBa}_{2} \mathrm{Cu}_{3} \mathrm{O}_{7-x}$ Thin Films," 1995 March Meeting of the American Physical Society, San Jose, CA, 20-24 March 1995. 